\title{
Limits and Degenerations of Unitary Conformal Field Theories
}

\author{
Daniel Roggenkamp ${ }^{1,2}$, Katrin Wendland ${ }^{3}$ \\ 1 Physikalisches Institut, Universität Bonn, Nußallee 12, D-53115 Bonn, Germany. \\ E-mail: roggenka@th.physik.uni-bonn.de \\ 2 Department of Mathematics, King's College London, Strand, London WC2R 2LS, United \\ Kingdom. \\ 3 Mathematics Institute, University of Warwick, Coventry CV4-7AL, United Kingdom. \\ E-mail: wendland@maths.warwick.ac.uk
}

\begin{abstract}
In the present paper, degeneration phenomena in conformal field theories are studied. For this purpose, a notion of convergent sequences of CFTs is introduced. Properties of the resulting limit structure are used to associate geometric degenerations to degenerating sequences of CFTs, which, as familiar from large volume limits of non-linear sigma models, can be regarded as commutative degenerations of the corresponding "quantum geometries".

As an application, the large level limit of the A-series of unitary Virasoro minimal models is investigated in detail. In particular, its geometric interpretation is determined.
\end{abstract}

\section{Introduction}

Limits and degenerations of conformal field theories (CFTs) have occurred in various ways in the context of compactifications of moduli spaces of CFTs, in particular in connection with string theory. For example, zero curvature or large volume limits of CFTs that correspond to sigma models are known to give boundary points of the respective moduli spaces $\mathrm{A}-\mathrm{G}-\mathrm{M}$ Mo. These limits provide the connection between string theory and classical geometry which for instance is used in the study of D-branes. In the Strominger/Yau/Zaslow mirror construction $\mathrm{V}-\mathrm{W} \mid \mathrm{S}-\mathrm{Y}-\mathrm{Z} \mathrm{Gr}$, boundary points play a prominent role. In fact, Kontsevich and Soibelman have proposed a mirror construction on the basis of the Strominger/Yau/Zaslow conjecture which relies on the structure of the boundary of certain CFT moduli spaces $\underline{\mathrm{K}-\mathrm{S}}$.

All the examples mentioned above feature interesting degeneration phenomena. Namely, subspaces of the Hilbert space which are confined to be finite dimensional for a well-defined CFT achieve infinite dimensions in the limit. In fact, such degenerations are expected if the limit is formulated in terms of non-linear sigma models, where at large volume, the algebra of low energy observables is expected to yield a non-commutative deformation of an algebra $\mathcal{A}^{\infty}$ of functions 
on the target space. The algebra of observables whose energy converges to zero then reduces to $\mathcal{A}^{\infty}$ at infinite volume. An entire non-commutative geometry can be extracted from the underlying CFT, which approaches the target space geometry in the limit $[\mathrm{F}-\mathrm{G}$. By construction, this formulation should encode geometry in terms of Connes' spectral triples $\mathrm{Co1}$ Co2 $\mathrm{Co3}$.

By the above, degeneration phenomena are crucial in order to single out an algebra which encodes geometry in CFTs. An intrinsic understanding of limiting processes in CFT language is therefore desirable. This will also be necessary in order to take advantage of the geometric tools mentioned before, away from those limits. Vice versa, a good understanding of such limiting processes in CFTs could allow to take advantage of the rich CFT structure in geometry.

The main aim of the present work is to establish an intrinsic notion of such limiting processes in pure CFT language and to apply it to some interesting examples. To this end, we give a definition of convergence for sequences of CFTs, such that the corresponding limit has the following structure: There is a limiting pre-Hilbert space $\mathcal{H}^{\infty}$ which carries the action of a Virasoro algebra, and similar to ordinary CFTs to each state in $\mathcal{H}^{\infty}$ we assign a tower of modes. Under an additional condition the limit even has the structure of a full CFT on the sphere. This is the case in all known examples, and in particular, our notion of limiting processes is compatible with deformation theory of CFTs.

If the limit of a converging sequence of CFTs has the structure of a CFT on the sphere, but is not a full CFT, then this is due to a degeneration as mentioned above. In particular, the degeneration of the vacuum sector can be used to read off a geometry from such a degenerate limit. Namely, in our limits the algebra of zero modes assigned to those states in $\mathcal{H}^{\infty}$ with vanishing energy is commutative and can therefore be interpreted as algebra of smooth functions on some manifold $M$. The asymptotic behaviour of the associated energy eigenvalues allows to read off a degenerating metric on $M$ and an additional smooth function corresponding to the dilaton as well. Moreover, being a module of this commutative algebra, $\mathcal{H}^{\infty}$ can be interpreted as a space of sections of a sheaf over $M$ as is explained in $[\mathrm{K}-\mathrm{S}]$.

Simple examples which we can apply our techniques to are the torus models, where our limit structure yields geometric degenerations of the corresponding target space tori à la Cheeger-Gromov C-G1,C-G2. In this case, $\mathcal{H}^{\infty}$ is the space of sections of a trivial vector bundle over the respective target space torus. Similar statements are true for orbifolds of torus models, only that in this case the fiber structure of $\mathcal{H}^{\infty}$ over the respective torus orbifold is non-trivial. Namely, the twisted sectors contribute sections of skyscraper sheaves localized on the orbifold fixed points.

Our favorite example, which in fact was the starting point of our investigations, is the family of unitary Virasoro minimal models. Some of their structure constants have a very regular behaviour under the variation of the level of the individual models. We use this to show that the A-series of unitary Virasoro minimal models constitutes a convergent sequence of CFTs. All fields in its limit theory at infinite level can be constructed in terms of operators in the $\mathfrak{s u}(2)_{1}$ WZW model. The sequence degenerates, and the limit has a geometric interpretation in the above sense on the interval $[0, \pi]$ equipped with the (dilaton-corrected) metric $g(x)=\frac{4}{\pi^{2}} \sin ^{4} x$ (in fact, the $x$-dependent contribution is entirely due to the dilaton). This also allows us to read off the geometry of D-branes in these 
models. Though this means that the vacuum sector of our limit is well understood, it remains an interesting open problem to investigate the full fusion rules in detail, in particular whether an appropriate limiting S-matrix can be found.

A different limit for the A-series of unitary Virasoro minimal models at infinite level was proposed in G-R-W R-W1 R-W2. It is described by a well-defined non-rational CFT of central charge one, which bears some resemblance to Liouville theory. In particular, its spectrum is continuous, but degenerations do not occur. Our techniques can also be used to describe this latter limit. The relation between the two different limit structures is best compared to the case of a free boson, compactified on a circle of large radius, where apart from the degenerate limit described above one can also obtain the decompactified free boson. While the limit investigated in this article has the advantage that it leads to a consistent geometric interpretation, the one which corresponds to the decompactified free boson gives a new well-defined non-rational CFT.

This work is organized as follows: In Sect. 1]we explain how non-commutative geometries can be extracted from CFTs, after giving a brief overview of some of the basic concepts. Sect. 2 contains our definitions of sequences, convergence, and limits, and is the technical heart of this paper. Moreover, the geometric interpretations of degenerate limits are discussed. Sect. 3 is devoted to the study of torus models and orbifolds thereof, where we exemplify our techniques. In Sect. 4 we present our results on the A-series of unitary Virasoro minimal models. We end with a discussion in Sect. 5 . Several appendices contain background material and lengthy calculations.

Acknowledgments. It is a pleasure to thank Gavin Brown, Jarah Evslin, José Figueroa-O'Farrill, Matthias Gaberdiel, Maxim Kontsevich, Werner Nahm, Andreas Recknagel, Michael Rösgen, Volker Schomerus, Gérard Watts and the referee for helpful comments or discussions. We also wish to thank the "abdus salam international center for theoretical physics" for hospitality, since part of this work was performed there.

D. R. was supported by DFG Schwerpunktprogramm 1096 and by the Marie Curie Training Site "Strings, Branes and Boundary Conformal Field Theory" at King's College London, under EU grant HPMT-CT-2001-00296. K. W. was partly supported under U.S. DOE grant DE-FG05-85ER40219, TASK A, at the University of North Carolina at Chapel Hill.

\section{From geometry to conformal field theory, and back to geometry}

String theory establishes a natural map which associates CFTs to certain, sometimes degenerate geometries. Conversely, one can associate a GEOMETRIC INTERPRETATION to certain CFTs, and the latter construction is made precise by using Connes' definition of spectral triples and non-commutative geometry.

In Sect. 1.1 we very briefly remind the reader of SPECTRAL TRIPLES, explaining how they encode geometric data. Somewhat relaxing the conditions on spectral triples we define SPECTRAL PRE-TRIPLES which will be used in Sect. 1.2 There, we recall the basic structure of CFTs and show how to extract spectral pre-triples from them. If the spectral pre-triple defines a spectral triple, then this will generate a non-commutative geometry from a given CFT. In Sect. 1.3 we explain how in favorable cases we can generate commutative geometries from 
CFTs. In the context of string theory, this prescription gives back the original geometric data of the compactification space.

Much of this Sect. 1 consists of a summary of known results [Co2 [F-G] $\mathrm{Re}, \mathrm{K}-\mathrm{S}$, but it also serves to introduce our notations.

1.1. From Riemannian geometry to spectral triples. For a compact Riemannian manifold $(M, g)$, which for simplicity we assume to be smooth and connected, the spectrum of the associated Laplace-Beltrami operator $\Delta_{g}$ : $C^{\infty}(M) \longrightarrow C^{\infty}(M)$ encodes certain geometric data of $(M, g)$. However, in general one cannot hear the shape of a drum, and more information than the set of eigenvalues of $\Delta_{g}$ is needed in order to recover $(M, g)$.

By the Gel'fand-Naimark theorem, the point set topology of $M$ is completely encoded in $C^{0}(M)=\overline{C^{\infty}(M)}$ : We can recover each point $p \in M$ from the ideal of functions which vanish at $p$. In other words, given the structure of $C^{\infty}(M)$ as a $\mathbb{C}^{*}$-algebra and its completion $C^{0}(M), M$ is homeomorphic to the set of closed points of $\operatorname{Spec}\left(\mathcal{O}_{M}\right)$, where $\mathcal{O}_{M}$ is the sheaf of regular functions on $M$. Connes' dual prescription uses $\mathbb{C}^{*}$-algebra homomorphisms $\chi: C^{\infty}(M) \longrightarrow \mathbb{C}$, instead, such that $p \in M$ corresponds to $\chi_{p}: C^{\infty}(M) \longrightarrow \mathbb{C}$ with $\chi_{p}(f):=f(p)$; the Gel'fand-Naimark theorem ensures that for every commutative $\mathbb{C}^{*}$-algebra $\overline{\mathcal{A}}$ there exists a Hausdorff space $M$ with $\overline{\mathcal{A}}=C^{0}(M)$. $M$ is compact if $\overline{\mathcal{A}}$ is unital.

Example 1.1.1

Let $R \in \mathbb{R}^{+}$, then $M=\mathbb{S}_{R}^{1}=\mathbb{R}^{1} / \sim$ with coordinate $x \sim x+2 \pi R$ has the Laplacian $\Delta=-\frac{d^{2}}{d x^{2}}$. Its eigenfunctions $|m\rangle_{R}, m \in \mathbb{Z}$, obey

$$
\begin{aligned}
& \forall m \in \mathbb{Z}: \quad|m\rangle_{R}: x \mapsto e^{i m x / R} ; \quad \frac{1}{2} \Delta|m\rangle_{R}=\frac{m^{2}}{2 R^{2}}|m\rangle_{R} ; \\
& \forall m, m^{\prime} \in \mathbb{Z}: \quad|m\rangle_{R} \cdot\left|m^{\prime}\right\rangle_{R}=\left|m+m^{\prime}\right\rangle_{R},
\end{aligned}
$$

and they form a basis of $C^{0}(M)$ and $C^{\infty}(M)$ with respect to the appropriate norms. Any smooth manifold is homeomorphic to $\mathbb{S}_{R}^{1}$, equipped with the Zariski topology, if its algebra of continuous functions has a basis $f^{m}, m \in \mathbb{Z}$, which obeys the multiplication law $f^{m} \cdot f^{m^{\prime}}=f^{m+m^{\prime}}$.

To recover the Riemannian metric $g$ on $M$ as well, we consider the SPECTRAL TRIPLE $\left(\mathbb{H}:=L^{2}\left(M, \mathrm{dvol}_{g}\right), H:=\frac{1}{2} \Delta_{g}, \mathcal{A}:=C^{\infty}(M)\right)$, where $H$ is viewed as self-adjoint operator which is densely defined on the Hilbert space $\mathbb{H}$, and $\mathcal{A}$ is interpreted as algebra of bounded operators which act on elements of $\mathbb{H}$ by pointwise multiplication. Following [Co2 $|\mathrm{F}-\mathrm{G}| \mathrm{Co} 3$, we can define a distance functional $d_{g}$ on the topological space $M$ by considering

$$
\begin{aligned}
\mathcal{F}:=\left\{f \in \mathcal{A} \mid G_{f}:=\right. & {[f,[H, f]]=-\left(f^{2} \circ H+H \circ f^{2}\right)+2 f \circ H \circ f } \\
& \text { obeys } \left.\forall h \in C^{\infty}(M):\left|G_{f} h\right| \leq|h|\right\} .
\end{aligned}
$$

One now sets

$$
\forall x, y \in M: \quad d_{g}(x, y):=\sup _{f \in \mathcal{F}}|f(x)-f(y)| .
$$

In Ex. 1.1.1 with $M=\mathbb{S}_{R}^{1}$ one checks that for all $f, h \in C^{\infty}(M): G_{f} h=\left(f^{\prime}\right)^{2} h$, and in general $G_{f} h=g(\nabla f, \nabla f) h$. In fact, by definition [B-G-V Prop. 2.3], 
any second-order differential operator $O$ satisfying $\left[f,\left[\frac{1}{2} O, f\right]\right]=g(\nabla f, \nabla f)$ is a GENERALIZED LAPLACIAN. Using the time coordinate of a geodesic from $x$ to $y$ and truncating and smoothing it appropriately one checks that (1.1.2) indeed gives back the geodesic distance between $x$ and $y$ which corresponds to the metric $g$. In other words, $(M, g)$ can be recovered from the spectral triple $(\mathbb{H}, H, \mathcal{A})$.

More generally, consider a spectral triple $(\mathbb{H}, H, \mathcal{A})$ with $\mathbb{H}$ a Hilbert space, $H$ a self-adjoint positive semi-definite operator, which on $\mathbb{H}$ is densely defined with $\mathcal{H}_{0,0}:=\operatorname{ker}(H) \cong \mathbb{C}$, and $\mathcal{A}$ a $\mathbb{C}^{*}$-algebra of bounded operators acting on $\mathbb{H}$. In fact, in the above let us assume that $M$ is spin and replace $H=\frac{1}{2} \Delta_{g}$ by the corresponding Dirac operator $\mathcal{D}$ and $\mathbb{H}=L^{2}\left(M, \operatorname{dvol}_{g}\right)$ by the Hilbert space $\mathbb{H}^{\prime}$ of square-integrable sections of the spinor bundle on $M$. Note that $H$ can be calculated from $\mathcal{D}$, see (1.1.3) and (1.1.5). Moreover, we assume that $\left(\mathbb{H}^{\prime}, \mathcal{D}, \mathcal{A}\right)$ obeys the SEven axioms of non-Commutative GeOmetry Co3. p.159]. Roughly speaking, these axioms ensure that the eigenvalues of $H$ have the correct growth behaviour (1.1.4), that $\mathcal{D}$ defines a map $\nabla$ on $\mathcal{A}$ with

$$
\forall f \in \mathcal{A}: \quad \nabla f:=[\mathcal{D}, f]: \mathbb{H}^{\prime} \rightarrow \mathbb{H}^{\prime} ; \quad \forall h \in \mathcal{A}: \quad[\nabla f, h]=0,
$$

where in the above examples $\nabla f$ acts on $\mathbb{H}^{\prime}$ by Clifford multiplication, and that $\mathcal{A}$ gives smooth coordinates on an "orientable geometry"; furthermore, there are finiteness and reality conditions as well as a type of Poincaré duality on the Kgroups of $\mathcal{A}$. If all these assumptions hold, then by (1.1.2) the triple $\left(\mathbb{H}^{\prime}, \mathcal{D}, \mathcal{A}\right)$ defines a non-commutative geometry à la Connes Co1 Co2 Co3. If the algebra $\mathcal{A}$ is commutative, then the triple $\left(\mathbb{H}^{\prime}, \mathcal{D}, \mathcal{A}\right)$ in fact defines a unique ordinary Riemannian geometry $(M, g)$ Co3 p.162]. The claim that the differentiable and the spin structure of $(M, g)$ can be fully recovered is detailed in ${ }^{1} \mathrm{Re}$.

Following [F-G], instead of studying SPECTRAL TRIPLES $\left(\mathbb{H}^{\prime}, \mathcal{D}, \mathcal{A}\right)$, we will be less ambitious and mainly focus on triples $(\mathbb{H}, H, \mathcal{A})$, somewhat relaxing the defining conditions:

\section{Definition 1.1.2}

We call $(\mathbb{H}, H, \mathcal{A})$ a SPECTRAL PRE-TRIPLE if $\mathbb{H}$ is a pre-Hilbert space over $\mathbb{C}, H$ is a self-adjoint positive semi-definite operator on $\mathbb{H}$ with $\mathcal{H}_{0,0}:=\operatorname{ker}(H) \cong \mathbb{C}$, and $\mathcal{A}$ is an algebra of operators acting on $\mathbb{H}$. Since $\mathcal{H}_{0,0} \cong \mathbb{C} \ni 1$, we can view $\mathcal{A} \hookrightarrow \mathbb{H}$ by $A \mapsto A \cdot 1$.

If additionally the eigenvalues of $H$ have the appropriate growth behaviour, i.e. for some $\gamma \in \mathbb{R}$ and $V \in \mathbb{R}$ :

$$
N(E):=\operatorname{dim}_{\mathbb{C}}\left(\bigoplus_{\lambda \leq E}\{\varphi \in \mathbb{H} \mid H \varphi=\lambda \varphi\}\right), \quad N(E) \stackrel{E \rightarrow \infty}{\sim} V \cdot E^{\gamma / 2},
$$

then $(\mathbb{H}, H, \mathcal{A})$ is called a SPECTRAL PRE-TRIPLE OF DIMENSION $\gamma$.

If there exists an operator $\mathcal{D}$ which is densely defined on a Hilbert space $\mathbb{H}^{\prime}$ that carries an action of $\mathcal{A}$ with (1.1.3) such that

$$
\forall f, h \in \mathcal{A}: \quad\langle\nabla f, \nabla h\rangle_{\mathbb{H}^{\prime}}=2\langle f, H h\rangle_{\mathbb{H}}
$$

and such that $\left(\mathbb{H}^{\prime}, \mathcal{D}, \mathcal{A}\right)$ obeys the seven axioms of non-commutative geometry, then we call $(\mathbb{H}, H, \mathcal{A})$ a SPECTRAL TRIPLE or a NON-COMMUTATIVE GEOMETRY

\footnotetext{
1 We thank Diarmuid Crowley for bringing this paper to our attention.
} 
OF DIMENSION $\gamma$.

\section{Remark 1.1.3}

Note that our condition (1.1.5) for the operator $H$ does not imply $H=\frac{1}{2} \Delta_{g}$ on $L^{2}\left(M, \operatorname{dvol}_{g}\right)$. In fact, $H$ will in general be a generalized Laplacian with respect to a metric $\widetilde{g}=\left(\widetilde{g}_{i j}\right)$ in the conformal class of $g$. More precisely, we will have $\operatorname{dvol}_{g}=e^{2 \Phi} \operatorname{dvol}_{\tilde{g}}$ with $\Phi \in C^{\infty}(M)$, and with $\widetilde{g}^{-1}=\left(\widetilde{g}^{i j}\right)$,

$$
2 H=-e^{-2 \Phi} \sqrt{\operatorname{det} \widetilde{g}^{-1}} \sum_{i, j} \partial_{i} e^{2 \Phi} \sqrt{\operatorname{det} \widetilde{g}} \widetilde{g}^{i j} \partial_{j}
$$

with respect to local coordinates, in accord with (1.1.5). We call $g$ the DILATON CORRECTED METRIC with DILATON $\Phi$. Note that $\widetilde{g}$ is easily read off from the symbol of $H$, allowing to determine $\Phi$ from $\operatorname{dvol}_{g}=e^{2 \Phi} \operatorname{dvol}_{\tilde{g}}$.

A generalization of Connes' approach, which is natural from our point of view, is given in $\mathrm{Lo}$. There, the Dirac operator on the spinor bundle of $M$ is replaced by the Dirac type operator $\mathcal{D}=d+d^{*}$ on $\mathbb{H}^{\prime}=\Lambda^{*}\left(T^{*} M\right)$. Since $\Delta_{g}=\mathcal{D}^{2}$, (1.1.3)11.1.5) remain true, but the list of axioms reduces considerably to the definition of a RiEmannian NON-COMmUTATIVE GEOMETRY [LO, III.2]. However, our main emphasis lies on the recovery of the metric structure $(M, g)$ rather than the differentiable structure. Similarly, in $\mathrm{K}-\mathrm{S}$ the main emphasis lies on triples $\left(M, \mathbb{R}^{+} g, \varphi\right)$, where $\varphi: M \longrightarrow \mathcal{M}$ is a map into an appropriate moduli space $\mathcal{M}$ of CFTs.

It will be easy to associate a spectral pre-triple to every CFT. Using degenerations of CFTs in the spirit of [K-S], one can often associate spectral pre-triples of dimension $\gamma=c$ to a CFT with central charge $c$. A general theorem, however, which allows to associate non-commutative geometries to arbitrary CFTs seems out of reach. In all cases we are aware of where a non-commutative geometry is obtained from CFTs, this is in fact proven by deforming an appropriate com-

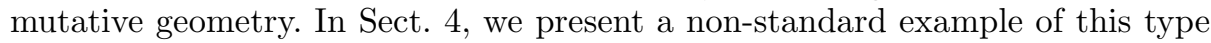
which should lead to interesting non-commutative geometries by deformation.

1.2. Spectral triples from CFTs. We do not attempt to give a complete definition of CFTs in this section; the interested reader may consult, e.g., B-P-Z M-S2, Gi $\mid$ M-S1 F-M-S G-G]. Some further properties of CFTs that are needed in the main text are collected in App. A

A UNITARY TWO-DIMENSIONAL CONFORMAL FIELD THEORY (CFT) is specified by the following data:

- a $\mathbb{C}$-vector space $\mathcal{H}$ of STATES with scalar product $\langle\cdot \mid \cdot\rangle$. This scalar product is positive definite, since we restrict our discussion to unitary CFTs;

- an anti-C-linear involution $*$ on $\mathcal{H}$, often called ChARGE CONJUGATION;

- an action of two commuting copies $\mathcal{V i r}_{c}, \overline{\mathcal{V i r}}_{c}$ of a Virasoro algebra A.1 with central charge $^{2} c \in \mathbb{R}$ on $\mathcal{H}$, with generators $L_{n}, \bar{L}_{n}, n \in \mathbb{Z}$, which ${ }^{3}$ commutes

\footnotetext{
2 As a matter of convenience, we always assume left and right handed central charges to agree.

3 The indexing of all modes below corresponds to energy, not to its negative.
} 
with $*$. The Virasoro generators $L_{0}$ and $\bar{L}_{0}$ are diagonalizable on $\mathcal{H}$, such that $\mathcal{H}$ decomposes into eigenspaces ${ }^{4}$

$$
\mathcal{H}=\bigoplus_{\substack{h, \bar{h} \in \mathbb{R}, h-\bar{h} \in \mathbb{Z}}} \mathcal{H}_{h, \bar{h}},
$$

and we set $\mathcal{H}_{h, \bar{h}}:=\{0\}$ if $h-\bar{h} \notin \mathbb{Z}$. The decomposition (1.2.1) is orthogonal with respect to $\langle\cdot \mid \cdot\rangle$;

- a GROWTH CONDITION for the eigenvalues $h, \bar{h}$ in (1.2.1): For some $\nu \in \mathbb{R}^{+}$ and $V \in \mathbb{R}$ :

$$
\forall E \in \mathbb{R}^{+}: \quad \infty \quad>\operatorname{dim}\left(\bigoplus_{(h+\bar{h})^{\nu} \leq E} \mathcal{H}_{h, \bar{h}}\right) \stackrel{E \rightarrow \infty}{\sim} \exp (V \sqrt{E}) .
$$

In particular, for all $h, \bar{h} \in \mathbb{R}$ we have $\mathcal{H}_{h, \bar{h}}^{*} \cong \mathcal{H}_{h, \bar{h}}$, and we define

$$
\check{\mathcal{H}}^{*}:=\bigoplus_{h, \bar{h} \in \mathbb{R}} \mathcal{H}_{h, \bar{h}}^{*}
$$

- a unique $*$-invariant VACUUM $\Omega \in \mathcal{H}_{0,0} \cong \mathbb{C}$, as well as a dual $\Omega^{*} \in \check{\mathcal{H}}^{*}$ characterized by (A.2);

- a map $C: \check{\mathcal{H}}^{*} \otimes \mathcal{H}^{\otimes 2} \longrightarrow \mathbb{C}$ that encodes the COEFFICIENTS OF THE OPERATOR PRODUCT EXPANSION (OPE), such that

$$
C(\cdot, \Omega, \cdot): \check{\mathcal{H}}^{*} \otimes \mathcal{H} \longrightarrow \mathbb{C}, \quad(\Psi, \chi) \longmapsto \Psi(\chi),
$$

i.e. the induced map is the canonical pairing. The OPE-coefficients $C$ obey (A.10) and (A.12) and can be used to define an isomorphism

$$
\begin{gathered}
\mathcal{H} \longrightarrow \check{\mathcal{H}}^{*} \\
\psi \longmapsto \psi^{*}
\end{gathered}, \quad \text { s. th. } \forall \chi \in \mathcal{H}: \quad \psi^{*}(\chi)=C\left(\psi^{*}, \Omega, \chi\right)=\langle\psi \mid \chi\rangle .
$$

There are many properties of the map $C$, like the sewing relations, that have to be fulfilled for reasons of consistency, and which we will not indulge to list explicitly. Some properties of CFTs that follow from these consistency conditions should be kept in mind, however:

$-\varphi \in \mathcal{H}$ is a lowest weight vector (lwv) with respect to the action of $\mathcal{V i r}_{c}, \overline{\mathcal{V i r}}_{c}$, i.e. a PRIMARY STATE, iff for $\operatorname{all}^{5} n \in \mathbb{N}-\{0\}: L_{-n} \varphi=0, \bar{L}_{-n} \varphi=0$.

For any $\mathbb{Z}$-graded algebra $\mathcal{L}=\bigoplus_{n \in \mathbb{Z}} \mathcal{L}_{n}$ we define

$$
\begin{aligned}
\mathcal{L}^{ \pm} & :=\bigoplus_{ \pm n>0} \mathcal{L}_{n} \\
\mathcal{H}^{\mathcal{L}}:=\quad \operatorname{ker} \mathcal{L}^{-} & =\left\{\varphi \in \mathcal{H} \mid \forall n \in \mathbb{N}-\{0\}, \forall w \in \mathcal{L}_{-n}: w \varphi=0\right\} .
\end{aligned}
$$

In other words, setting $\mathcal{L}=\mathcal{V}$ ir $=\mathcal{V}_{i r} \oplus \overline{\mathcal{V i r}}_{c}$ by abuse of notation, $\mathcal{H}^{\mathcal{V i r}}$ denotes the SUbSPACE OF PRIMARY STATES IN $\mathcal{H}$.

\footnotetext{
${ }^{4}$ In this work, we restrict our investigations to bosonic CFTs.

${ }^{5}$ We agree on $0 \in \mathbb{N}$, as argued in Bo IV.4.1,R.6.2].
} 
- The OPE, which we encode in the map $C$ as introduced above, allows to associate to each $\varphi \in \mathcal{H}$ a tower $\varphi_{\mu, \bar{\mu}}, \mu, \bar{\mu} \in \mathbb{R}$, of linear operators $\varphi_{\mu, \bar{\mu}}: \mathcal{H}_{h, \bar{h}} \longrightarrow$ $\mathcal{H}_{h+\mu, \bar{h}+\bar{\mu}}$, called (FOURIER) MODES, see A.13). In particular, the elements $L_{n}, \bar{L}_{n}, n \in \mathbb{Z}$, of $\mathcal{V}_{\mathrm{ir}_{c}}, \overline{\mathcal{V i r}}_{c}$ can be interpreted as the Fourier modes of the holomorphic and antiholomorphic parts $T, \bar{T}$ of the ENERGY-MOMENTUM TENSOR. Moreover, $\Omega_{0,0}$ acts as identity on $\mathcal{H}$, and all other modes of $\Omega$ act by multiplication with zero. By abuse of notation we write $T=L_{2} \Omega \in \mathcal{H}_{2,0}$, $\bar{T}=\bar{L}_{2} \Omega \in \mathcal{H}_{0,2}$ for the Virasoro states in $\mathcal{H}$.

A sextuple $\mathcal{C}=(\mathcal{H}, *, \Omega, T, \bar{T}, C)$ with $\mathcal{H}, *, \Omega, T, \bar{T}, C$ as above specifies a CFT. Two CFTs $\mathcal{C}=(\mathcal{H}, *, \Omega, T, \bar{T}, C)$ and $\mathcal{C}^{\prime}=\left(\mathcal{H}^{\prime}, *^{\prime}, \Omega^{\prime}, T^{\prime}, \bar{T}^{\prime}, C^{\prime}\right)$ are EQUIVALENT, if there exists a vector space homomorphism $I: \mathcal{H} \longrightarrow \mathcal{H}^{\prime}$, such that $I:(\Omega, T, \bar{T}) \mapsto\left(\Omega^{\prime}, T^{\prime}, \bar{T}^{\prime}\right)$ and $*^{\prime}=I \circ *, C^{\prime}=C \circ\left(I^{*} \otimes I \otimes I\right)$.

Instead of primary states in $\mathcal{H}^{\mathcal{V i r}}$, below, we will be interested in primary states with respect to a larger algebra than $\mathcal{V}$ ir, namely the (generic) HOLOMORPHic AND ANTiHolomorphic W-Algebra $\mathcal{W}^{*} \oplus \overline{\mathcal{W}}^{*}$, see (A.15). By (1.2.5) the primary states with respect to a subalgebra $\mathcal{W}$ of $\mathcal{W}^{*} \oplus \overline{\mathcal{W}}^{*}$ are

$$
\mathcal{H}^{\mathcal{W}}=\operatorname{ker} \mathcal{W}^{-}=\left\{\varphi \in \mathcal{H} \mid \forall n \in \mathbb{N}-\{0\}, \forall w \in \mathcal{W}_{-n}: w \varphi=0\right\} .
$$

To TRUNCATE THE OPE TO PRIMARIES note that by (1.2.2) for given $\varphi \in \mathcal{H}$ and $\chi \in \mathcal{H}_{h, \bar{h}}$, we have $\varphi_{\mu, \bar{\mu}} \chi \neq 0$ for a discrete set of $(\mu, \bar{\mu}) \in \mathbb{R}^{2}$. Hence, whenever the set

$$
I_{\mathcal{W}}(\varphi, \chi):=\left\{(\mu, \bar{\mu}) \in \mathbb{R}^{2} \mid \exists \psi \in \mathcal{H}^{\mathcal{W}}: \psi^{*}\left(\varphi_{\mu, \bar{\mu}} \chi\right) \neq 0\right\}
$$

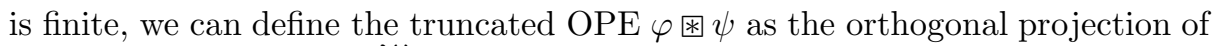

$$
\begin{aligned}
& \sum_{(\mu, \bar{\mu}) \in I_{\mathcal{W}}(\varphi, \chi)} \varphi_{\mu, \bar{\mu}} \chi \text { onto } \mathcal{H}^{\mathcal{W}}: \\
& \mathcal{H}^{\mathcal{W}}:=\left\{\varphi \in \mathcal{H}^{\mathcal{W}}\left|\forall \chi \in \mathcal{H}^{\mathcal{W}}:\right| I_{\mathcal{W}}(\varphi, \chi) \mid<\infty\right\} ; \\
& \forall \varphi \in \mathcal{H}^{\mathcal{W}}, \forall \chi \in \mathcal{H}^{\mathcal{W}}: \\
& \varphi \text { 国 } \chi \in \mathcal{H}^{\mathcal{W}} \text { s.th. } \forall \psi \in \mathcal{H}^{\mathcal{W}}: \psi^{*}(\varphi \text { 圈 } \chi)=C\left(\psi^{*}, \varphi, \chi\right) .
\end{aligned}
$$

Let us remark that the above definition of $⿴$ may well be too restrictive: By introducing appropriate (partial) completions of the relevant vector spaces one can attempt to replace our finiteness condition in 1.2.6) by a condition on normalizability and thereby get rid of the restriction to $\mathcal{H}^{\mathcal{W}}$. Although in most of our examples we find $\mathcal{H}^{\mathcal{W}}=\mathcal{H}^{\mathcal{W}}$, for the orbifolds discussed in Sect. $3.2 \mathcal{H}^{\mathcal{W}}-$ $\mathcal{H}^{\mathcal{W}}$ contains all twisted ground states. The latter do not enter into the discussion of the zero mode algebra, which is relevant for finding geometric interpretations (see Sect. 2.2). Summarizing, our definition of 困, above, is well adapted to our purposes, though it may be too restrictive in general. By construction,

$$
\forall \varphi \in \mathcal{H}^{\mathcal{W}}: \quad \varphi \text { 困 } \Omega=\Omega \text { 娄 } \varphi=\varphi .
$$

Let us extract a spectral pre-triple from a $\mathrm{CFT} \mathcal{C}=(\mathcal{H}, *, \Omega, T, \bar{T}, C)$. By definition of the adjoint (see A.5), A.11), $L_{0}$ acts as self-adjoint operator on $\mathcal{H}$, and $L_{1}^{\dagger}=L_{-1}$. Moreover, $2 L_{0}=\left[L_{1}^{\dagger}, L_{1}\right]$ shows that $L_{0}$ is positive semidefinite, and similarly for $\bar{L}_{0}$. Therefore, to associate a spectral pre-triple to a 
CFT $\mathcal{C}$, we will always use $H:=L_{0}+\bar{L}_{0}$, which by the uniqueness of $\Omega$ obeys $\operatorname{ker}(H)=\mathcal{H}_{0,0} \cong \mathbb{C}$. Following $[\mathrm{F}-\mathrm{G}$, we let

$$
\widetilde{\mathbb{H}}:=\mathcal{H}^{\mathcal{W}}=\operatorname{ker} \mathcal{W}^{-}
$$

denote the space of primaries in $\mathcal{H}$ with respect to an appropriate subalgebra $\mathcal{W}$ of the holomorphic and antiholomorphic W-algebras. Moreover, to every $\varphi \in \mathcal{H}^{\mathcal{W}}$ we associate an operator $A_{\varphi}$ on $\widetilde{\mathbb{H}}$ which acts by the truncated OPE $\varphi$ * as in (1.2.6). The operators $A_{\varphi}, \varphi \in \mathcal{H}^{\mathcal{W}}$, generate our algebra $\widetilde{\mathcal{A}}$ with the obvious vector space structure and with composition of operators as multiplication:

$$
\forall \varphi \in \mathcal{H}^{\mathcal{W}}: A_{\varphi}: \widetilde{\mathbb{H}} \longrightarrow \widetilde{\mathbb{H}}, A_{\varphi}(\chi):=\varphi \text { 困 } \chi ; \quad \widetilde{\mathcal{A}}:=\left\langle A_{\varphi} \mid \varphi \in \mathcal{H}^{\mathcal{W}}\right\rangle .
$$

It is not hard to see that $(\widetilde{\mathbb{H}}, H, \widetilde{\mathcal{A}})$ obeys Def. 1.1 .2 thus defining a spectral pre-triple. As a word of caution, we remark that in general for $\varphi, \chi \in \mathcal{H}^{\mathcal{W}}$, $A_{\varphi} \circ A_{\chi} \neq A_{\varphi \text { 国 }}$.

Several other attempts to associate an algebra to a CFT can be found in the literature. Truncation of the OPE to leading terms, as suggested in [K-S 2.2], gives a straight-forward algebra structure but seems not to capture enough of the algebraic information encoded in the OPE: On the one hand, if all states in $\mathcal{H}^{\mathcal{W}}$ are given by simple currents, e.g. for the toroidal theories focused on in [K-S], then truncation of the OPE to leading terms is equivalent to our truncation (1.2.6). On the other hand, for the example that we present in Sect. [4 it is not, and we show how our truncation (1.2.6) gives a convincing geometric interpretation. For holomorphic vertex operator algebras, ZHU's COMMUTATIVE ALGEBRA is a commutative associative algebra which can be constructed using the normal ordered product by modding out by its associator (see $\mathrm{Zh}, \mathrm{B}-\mathrm{N} \| \mathrm{G}-\mathrm{N}]$ ), and it is isomorphic to the zero-mode algebra $\mathrm{B}-\mathrm{N}$. Although to our knowledge Zhu's commutative algebra has not been generalized to the non-holomorphic case, it is very likely that such a generalization would yield the same geometric interpretations for degenerate CFTs that we propose below; this is related to the fact that $A_{\varphi} \circ A_{\chi}=A_{\varphi \circledast \chi}$ holds for the relevant states in these degenerate CFTs, see Lemma 2.2.3 and Prop. 2.2.4 Summarizing, our truncation (1.2.6), which goes back to [F-G], seems to unite the useful aspects of both the truncation of the OPE to leading terms and Zhu's algebra.

An application of Tauber's theorem known as Kawamata's theorem Wi Thm. 4.2] shows that the growth condition (1.2.2) ensures the eigenvalues of $H$ to obey (1.1.4) for $\gamma=\nu$. In general, $\gamma$ will not coincide with the central charge $c$ of the CFT, but in many examples with integral $c$ we find $\gamma=2 c$, see e.g. Ex. 1.2.2 below. So far, we have shown:

Proposition 1.2.1

To any $C F T \mathcal{C}=(\mathcal{H}, *, \Omega, T, \bar{T}, C)$ of central charge $c$ which obeys (1.2.2) with $\gamma=\nu \in \mathbb{R}$, after the choice of a subalgebra $\mathcal{W}$ of the holomorphic and antiholomorphic $W$-algebras, we associate a triple

$$
\left(\widetilde{\mathbb{H}}:=\mathcal{H}^{\mathcal{W}}=\operatorname{ker} \mathcal{W}^{-}, H:=L_{0}+\bar{L}_{0}, \widetilde{\mathcal{A}}:=\left\langle A_{\varphi} \mid \varphi \in \mathcal{H}^{\circ} \mathcal{W}\right\rangle\right)
$$

Then $(\widetilde{\mathbb{H}}, H, \widetilde{\mathcal{A}})$ is a spectral pre-triple of dimension $\gamma=\nu$ as in Def. 1.1.2 
The operator $\nabla:=L_{1}+\bar{L}_{1}: \widetilde{\mathbb{H}} \rightarrow \mathcal{H}$ obeys 1.1.5 as well as a Leibniz rule. However, for general CFTs we are unable to show that $(\widetilde{\mathbb{H}}, H, \widetilde{\mathcal{A}})$ gives a spectral triple of a specific dimension, i.e. a non-commutative geometry according to Def. 1.1.2 $\widetilde{\mathcal{A}}$ need not, in general, act by bounded operators, and we are unable to check all seven axioms of Connes' or their reduction in [Lo], including the fact that $\widetilde{\mathcal{A}}$ is a $\mathbb{C}^{*}$-algebra. Neither are we aware of any attempt to do so in the literature, see also [F-G-R] for a discussion of some unsolved problems that this approach poses.

For toroidal CFTs, the above construction indeed gives a $\mathbb{C}^{*}$-algebra $\widetilde{\mathcal{A}}$ of bounded operators $\mathrm{F}-\mathrm{G}$. We illustrate this by

\section{Example 1.2.2}

Let $\mathcal{C}_{R}, R \in \mathbb{R}^{+}$, denote the CIRCLE THEORY AT RADIUS $R$, i.e. the CFT with central charge $c=1$ that describes a boson compactified on a circle $^{6}$ of radius $R$. All $\mathcal{C}_{R}$ possess a subalgebra $\mathcal{W}=\mathfrak{u}(1) \oplus \overline{\mathfrak{u}(1)}$ of the holomorphic and antiholomorphic W-algebra ${ }^{7}$ (see App. B), and the pre-Hilbert space $\mathcal{H}_{R}$ of $\mathcal{C}_{R}$ decomposes into irreducible representations of $\mathcal{W}$. The latter can be labeled by left- and right handed dimension and charge $h_{R}, Q_{R}$ and $\bar{h}_{R}, \bar{Q}_{R}$ of their lwvs, where $h_{R}=\frac{1}{2} Q_{R}^{2}, \bar{h}_{R}=\frac{1}{2} \bar{Q}_{R}^{2}$. The space of primaries of $\mathcal{C}_{R}$ with respect to $\mathcal{W}$ is

$$
\begin{aligned}
\widetilde{\mathbb{H}}:=\mathcal{H}^{\mathcal{W}}=\operatorname{span}_{\mathbb{C}}\left\{\left|Q_{R} ; \bar{Q}_{R}\right\rangle:=\left|\frac{Q_{R}^{2}}{2}, Q_{R}\right\rangle \otimes\left|\frac{\bar{Q}_{R}^{2}}{2}, \bar{Q}_{R}\right\rangle \mid\right. \\
\left.\exists m, n \in \mathbb{Z}: Q_{R}=\frac{1}{\sqrt{2}}\left(\frac{m}{R}+n R\right), \bar{Q}_{R}=\frac{1}{\sqrt{2}}\left(\frac{m}{R}-n R\right)\right\},
\end{aligned}
$$

see (B.4). To obtain the spectral pre-triple associated to $\mathcal{C}_{R}$ by Prop. 1.2.1 from $\widetilde{\mathbb{H}}=\mathcal{H}^{\mathcal{W}}$ we need to consider the truncated OPE (1.2.6). By (1.2.7) and (B.6), orthonormalizing the $\left|Q_{R} ; \bar{Q}_{R}\right\rangle$ as in $(\overline{B .3})$, we have

$$
\begin{aligned}
A_{\left|Q_{R} ; \bar{Q}_{R}\right\rangle} \circ A_{\left|Q_{R}^{\prime} ; \bar{Q}_{R}^{\prime}\right\rangle} & =(-1)^{\left(Q_{R}+\bar{Q}_{R}\right)\left(Q_{R}^{\prime}-\bar{Q}_{R}^{\prime}\right) / 2} A_{\left|Q_{R}+Q_{R}^{\prime} ; \bar{Q}_{R}+\bar{Q}_{R}^{\prime}\right\rangle} \\
& =A_{\left|Q_{R} ; \bar{Q}_{R}\right\rangle \circledast\left|Q_{R}^{\prime} ; \bar{Q}_{R}^{\prime}\right\rangle} .
\end{aligned}
$$

We see that $\stackrel{\mathcal{H}}{ }^{\mathcal{W}}=\mathcal{H}^{\mathcal{W}}$ and $\widetilde{\mathcal{A}}:=\left\langle A_{\varphi} \mid \varphi \in \mathcal{H}^{\mathcal{W}}\right\rangle$ is generated by the $A_{\varphi}$ with $\varphi \in \mathcal{H}^{\mathcal{W}}$ as a vector space, i.e. $\circledast$ defines an (associative!) product on $\mathcal{H}^{\mathcal{W}}$, which simplifies the situation considerably in comparison to the general case. The algebra $\widetilde{\mathcal{A}}$ is clearly non-commutative. It is a straight-forward non-commutative extension of the product (1.1.1) of the algebra of smooth functions on the circle, taking winding and momentum modes into account. In fact, $\widetilde{\mathcal{A}}$ is the twisted group algebra $\mathbb{C}_{\varepsilon}[\Gamma]$ of the $\mathfrak{u}(1) \oplus \overline{\mathfrak{u}(1)}$ - charge lattice

$$
\Gamma=\left\{\left(Q_{R} ; \bar{Q}_{R}\right)=\left(Q_{m, n} ; \bar{Q}_{m, n}\right)=\frac{1}{\sqrt{2}}\left(\left(\frac{m}{R}+n R\right) ;\left(\frac{m}{R}-n R\right)\right) \mid m, n \in \mathbb{Z}\right\}
$$

\footnotetext{
${ }^{6}$ Our normalizations are such that the boson compactified on a circle of radius $R=1$ is described by the $\mathfrak{s u}(2)_{1}$ WZW model.

7 To clear notations, our symbol $\mathfrak{g}$ always denotes the loop algebra associated to the Lie group $G$ with Lie algebra $g$, and $\mathfrak{g}_{k}$ denotes its central extension of level $k$.
} 
(see (B.4)), twisted by the two-cocycle $\varepsilon$ of (B.6), yielding a non-commutative generalization of the algebra of smooth functions on $\mathbb{S}_{R}^{1} \times \mathbb{S}_{1 / R}^{1}$. Moreover, one checks that $(\widetilde{\mathbb{H}}, H, \widetilde{\mathcal{A}})$ is a spectral pre-triple of dimension $2=2 c$, and we have

$$
\begin{aligned}
Q_{R}= & \frac{1}{\sqrt{2}}\left(\frac{m}{R}+n R\right), \bar{Q}_{R}=\frac{1}{\sqrt{2}}\left(\frac{m}{R}-n R\right) \\
& \Longrightarrow H\left|Q_{R} ; \bar{Q}_{R}\right\rangle=\left(h_{R}+\bar{h}_{R}\right)\left|Q_{R} ; \bar{Q}_{R}\right\rangle=\left(\frac{m^{2}}{2 R^{2}}+\frac{n^{2} R^{2}}{2}\right)\left|Q_{R} ; \bar{Q}_{R}\right\rangle
\end{aligned}
$$

in perfect agreement with (1.1.1).

1.3. Commutative (sub)-geometries. By Prop. 1.2.1 there is a spectral pre-triple associated to every CFT. However, this construction is not very satisfactory. Namely, it depends on the choice of a $\mathrm{W}$-subalgebra $\mathcal{W}$, and it does not allow us to extract a non-commutative geometry à la Connes in a straight-forward manner. Moreover, if we start e.g. with the one-dimensional Riemannian geometry $\left(\mathbb{S}_{R}^{1}, g\right)$ discussed in Ex. 1.1.1 from its associated CFT we read off a spectral pre-triple $(\widetilde{\mathbb{H}}, H, \widetilde{\mathcal{A}})$ of dimension 2 in Ex. 1.2.2 The original one-dimensional spectral pre-triple $\left(\mathbb{H}=L^{2}\left(\mathbb{S}_{R}^{1}, \operatorname{dvol}_{g}\right), H=\frac{1}{2} \Delta_{g}, \mathcal{A}=C^{\infty}\left(\mathbb{S}_{R}^{1}\right)\right)$ can of course be obtained from $(\widetilde{\mathbb{H}}, H, \widetilde{\mathcal{A}})$ by restriction:

$$
\begin{aligned}
\mathbb{H} & =\operatorname{span}_{\mathbb{C}}\left\{|m\rangle_{R} \mid m \in \mathbb{Z}\right\} \\
& \cong \operatorname{span}_{\mathbb{C}}\left\{\left|Q_{R} ; \bar{Q}_{R}\right\rangle \in \widetilde{\mathbb{H}} \mid Q_{R}=\bar{Q}_{R}=\frac{m}{\sqrt{2} R}, m \in \mathbb{Z}\right\}=\operatorname{ker}\left\langle\mathcal{W}^{-}, j_{0}-\bar{\jmath}_{0}\right\rangle,
\end{aligned}
$$

where $j_{0}, \bar{j}_{0}$ denote the zero modes of generators $j, \bar{j}$ of $\mathfrak{u}(1), \overline{\mathfrak{u}(1)}$ as in (B.1). In (1.2.10) we have checked that $H$ has the correct eigenvalues on the generators of $\mathbb{H}$. Also, by (1.2.8), 困 is associative and commutative on $\mathbb{H} \cong \operatorname{ker}\left\langle\mathcal{W}^{-}, j_{0}-\bar{\jmath}_{0}\right\rangle$, and $\mathcal{A} \cong\left\langle A_{\varphi} \mid \varphi \in \mathbb{H}\right\rangle$. This motivates

\section{Definition 1.3.1}

Let $\mathcal{C}$ denote a $C F T$ with central charge $c, \mathcal{W}$ a subalgebra of its holomorphic and antiholomorphic $W$-algebras, and $\left(\widetilde{\mathbb{H}}=\operatorname{ker} \mathcal{W}^{-}, H, \widetilde{\mathcal{A}}\right)$ the associated spectral pre-triple of dimension $\gamma$ as in Prop. 1.2.1 A spectral pre-triple $(\mathbb{H}, H, \mathcal{A})$ of dimension $c$ is called a GEOMETRIC INTERPRETATION OF $\mathcal{C}$ if $\mathbb{H} \subset \widetilde{\mathbb{H}}$, $\mathcal{A}=\left\langle\left. A_{\varphi}\right|_{\mathbb{H}} \mid \varphi \in \mathbb{H}\right\rangle$ is commutative, and if there are appropriate completions $\overline{\mathbb{H}}, \overline{\mathcal{A}}$ of $\mathbb{H}, \mathcal{A}$ such that $(\overline{\mathbb{H}}, H, \overline{\mathcal{A}})$ is a spectral triple of dimension $c$, i.e. $\overline{\mathbb{H}}=L^{2}\left(M, \operatorname{dvol}_{g}\right), H=\frac{1}{2} \Delta_{\tilde{g}}, \overline{\mathcal{A}}=C^{\infty}(M)$ for some Riemannian manifold $(M, g)$ of dimension $c$ and $\operatorname{dvol}_{g}=e^{2 \Phi} \operatorname{dvol}_{\tilde{g}}, \Phi \in C^{\infty}(M)$.

One checks that each CFT $\mathcal{C}_{R}, R \in \mathbb{R}^{+}$, of Ex. 1.2.2 has precisely two geometric interpretations $\left(\mathbb{H}_{R}^{ \pm}, H, \mathcal{A}_{R}^{ \pm}\right)$with $\overline{\mathbb{H}_{R}^{ \pm}}=\overline{\operatorname{ker}\left\langle\mathcal{W}^{-}, j_{0} \mp \bar{\jmath}_{0}\right\rangle} \cong L^{2}\left(\mathbb{S}_{R^{ \pm 1}}^{1}\right)$. The ambiguity is not a problem but a well-known virtue, since the $\operatorname{CFTs} \mathcal{C}_{R}$ and $\mathcal{C}_{R^{-1}}$ are equivalent according to the definition given on p. 8. On the other hand, the non-linear sigma model construction with target the geometric interpretation $\mathbb{S}_{R^{ \pm 1}}^{1}$ of $\mathcal{C}_{R}$ gives back the CFT $\mathcal{C}_{R}$, yielding the notion of GEOMETRIC INTERPRETATION introduced in Def. [1.3.1 very natural. For general $(M, g)$, however, a rigorous construction of a non-linear sigma model with target $M$ is problematic: Renormalization is necessary, and perturbative methods give good approximations only for $M$ with large volume. Hence we can only expect those properties 
of Ex. 1.2.2 to generalize which characterize $\mathcal{C}_{R}=\mathcal{C}_{R^{-1}}$ at $R^{ \pm 1} \gg 0$. In fact, $\mathbb{H}_{R}^{ \pm}$ is generated by the $\left|Q_{R^{ \pm 1}} ; \bar{Q}_{R^{ \pm 1}}\right\rangle \in \widetilde{\mathbb{H}}$ with $Q_{R^{ \pm 1}}= \pm \bar{Q}_{R^{ \pm 1}}=\frac{m}{\sqrt{2} R^{ \pm 1}}, m \in \mathbb{Z}$. Hence by (1.2.10) for $R^{ \pm 1} \gg 0$ the geometric interpretation $\left(\mathbb{H}_{R}^{ \pm}, H, \mathcal{A}_{R}^{ \pm}\right)$is obtained from $(\widetilde{\mathbb{H}}, H, \widetilde{\mathcal{A}})$ by restriction to those states in $\widetilde{\mathbb{H}}$ which at large volume retain bounded energy:

Definition 1.3.2

Let $\mathcal{C}$ denote a CFT (or a limit of a sequence of CFTs, see Def. 2.1.6) with associated spectral pre-triple $(\widetilde{\mathbb{H}}, H, \widetilde{\mathcal{A}})$ and geometric interpretation $(\mathbb{H} \subset \widetilde{\mathbb{H}}, H, \mathcal{A} \subset$ $\widetilde{\mathcal{A}})$. Assume that there is an $\varepsilon>0, \varepsilon \ll 1$, such that

$$
\mathcal{A}_{0}:=\left\langle\left. A_{\varphi}\right|_{\mathbb{H}}|\varphi \in \widetilde{\mathbb{H}},| H \varphi|\leq \varepsilon| \varphi \mid\right\rangle \quad \text { obeys } \quad \mathbb{H}=\operatorname{span}_{\mathbb{C}}\left(\mathcal{A}_{0}\left(\mathcal{H}_{0,0}\right)\right) .
$$

Then $\mathcal{C}$ is called DEGENERATE and $(\mathbb{H}, H, \mathcal{A})$ or $(M, g)$ with $\overline{\mathbb{H}}=L^{2}\left(M, \operatorname{dvol}_{g}\right)$, $H=\frac{1}{2} \Delta_{\tilde{g}}$ and $\overline{\mathcal{A}}=C^{\infty}(M)$ as in Def. 1.3.1 is called PREFERRED GEOMETRIC INTERPRETATION OF $\mathcal{C}$.

The preferred geometric interpretations are exactly those geometric interpretations proposed and studied in [F-G]. It is believed that a degenerate CFT $\mathcal{C}$ with preferred geometric interpretation $(M, g)$ in fact yields a degenerate Riemannian geometry $(M, g)$. More precisely, in [K-S], families $\mathcal{C}_{\varepsilon}$ of degenerate CFTs with $\varepsilon>0$ as in Def. 1.3.2 are studied as $\varepsilon \rightarrow 0$, where the preferred geometric interpretations $\left(M_{\varepsilon}, g_{\varepsilon}\right), \varepsilon>0$, all yield the same topological manifold $M_{\varepsilon} \cong M$. Then $\left(M, g_{\varepsilon}\right)_{\varepsilon \rightarrow 0}$ is believed to describe a Gromov-Hausdorff limit of a metric on $M$, where some cycles collapse while keeping the curvature bounded. Such limits of metrics have been studied in C-G1,C-G2]. In the physics literature, the limiting geometries which arise from degenerate CFTs are sometimes referred to as LARGE VOLUME LIMITS, see Mo for a useful account.

Since each collapse of cycles $\left(M, g_{\varepsilon}\right)_{\varepsilon \rightarrow 0}$ in C-G1 C-G2 gives a boundary point of the moduli space of Riemannian metrics on $M$, it is natural to use sequences $\left(\mathcal{C}_{\varepsilon}\right)_{\varepsilon \rightarrow 0}$ as above to construct corresponding boundary points of moduli spaces of CFTs. In a more general context, such a possibility was alluded to in $[\mathrm{K}-\mathrm{S}$. It presumes the definition of topological data on the families of CFTs under consideration:

Definition 1.3.3

A CFT-SPACE is given by the following data: A sheaf $\mathcal{S}$ over a topological Hausdorff space $\mathcal{M}$, such that for each $p \in \mathcal{M}, \mathcal{C}_{p}$ is a CFT with associated pre-Hilbert space $\mathcal{H}_{p}=\mathcal{S}_{p}$. Furthermore $\Omega, T, \bar{T}$ are global sections of $\mathcal{S}$, and all CFT-structures as e.g. OPE-coefficients, evaluated on local sections of $\mathcal{S}$, are continuous. If $\mathcal{M}$ is a $D$-dimensional variety, then $D$ is called the DIMENSION of the CFT-space $\mathcal{S}$.

If $\mathcal{S}$ is obtained as a deformation space of CFTs in the sense of conformal deformation theory, then $\mathcal{M}$ comes equipped with a metric, the Zamolodchikov metric, which induces a standard topology on $\mathcal{M}$ as well as flat connections on $\mathcal{S}$ [Sch $, \mathrm{Ka}, \mathrm{R}-\mathrm{S}-\mathrm{Z}]$. This is in particular true for the family $\left(\mathcal{C}_{R}\right)_{R \in \mathbb{R}^{+}}$studied in Ex. 1.2.2.

Intuitively, it is now clear how boundary points of CFT-spaces $\mathcal{S}$ over $\mathcal{M}$ could be constructed: One considers continuous paths $p:[0, \infty) \rightarrow \mathcal{M}$ giving rise to 
one-dimensional CFT-subspaces $\mathcal{S}_{\mid p}$ of $\mathcal{S}$ with $\left(\mathcal{S}_{\mid p}\right)_{t}=\mathcal{H}_{p(t)}$ for $t \in[0, \infty)$. If for $t \rightarrow \infty$ the CFT-structures of the $\mathcal{C}_{p(t)}$ converge in a suitable sense, e.g. as specified in Sect. 2 then the limit structure gives rise to a boundary point of the CFT-space $\mathcal{S}_{\mid p}$. If $\lim _{t \rightarrow \infty} p(t)=\bar{p} \in \mathcal{M}$, then the CFT-structures converge to the corresponding structures of $\mathcal{C}_{\bar{p}}$, and the boundary point of the CFT-subspace $\mathcal{S}_{\mid p}$ just corresponds to this CFT. If however $\bar{p} \in \overline{\mathcal{M}}-\mathcal{M}$, then the boundary point of the CFT-subfamily $\mathcal{S}_{\mid p}$ can be considered as boundary point of $\mathcal{S}$.

Moreover, assume that to each $\mathcal{C}_{p(t)}$ we can associate a spectral pre-triple $\left(\mathbb{H}_{p(t)}, H, \mathcal{A}_{p(t)}\right)$ obtained from an appropriate subspace of constant sections along $p$. If there is an $N \in \mathbb{N}$ such that for every constant section $\varphi$ from such a subspace one has $\left|H \varphi_{p(t)}\right| /\left|\varphi_{p(t)}\right| \stackrel{t \rightarrow \infty}{=} O\left(t^{-N}\right)$, and if all structure constants of $\left(\mathbb{H}_{p(t)}, H, \mathcal{A}_{p(t)}\right)$ converge for $t \rightarrow \infty$, then we obtain a limiting spectral pre-triple $\left(\mathbb{H}^{\infty}, H, \mathcal{A}^{\infty}\right)$. The above assumption that all eigenvalues of $H$ on $\mathbb{H}_{p(t)}$ converge with the same speed $O\left(t^{-N}\right)$ allows to define $H^{\infty}:=\lim _{t \rightarrow \infty} t^{N} H$ and should allow to read off a non-degenerate Riemannian geometry from $\left(\mathbb{H}^{\infty}, H^{\infty}, \mathcal{A}^{\infty}\right)$.

For now, instead of considering CFTs, let us stay in the regime of function spaces and inspect limits of commutative geometries in terms of spectral triples. This serves as a motivation for Sect. 2] and also leads to some ambiguities which should be kept in mind.

\section{Example 1.3.4}

We consider possible limiting procedures for the spectral triples $\left(\mathbb{H}_{R}=L^{2}\left(\mathbb{S}_{R}^{1}\right)\right.$, $\left.H=\frac{1}{2} \Delta, \mathcal{A}_{R}=C^{\infty}\left(\mathbb{S}_{R}^{1}\right)\right)$ as $R \rightarrow \infty$. By (1.1.1), each $\mathbb{H}_{R}$ is generated by $\oplus_{m \in \mathbb{Z}} V_{m}^{R}$ with $V_{m}^{R}:=\operatorname{span}_{\mathbb{C}}\left\{|m\rangle_{R}\right\}$ an eigenspace of $H$ with eigenvalue $\frac{m^{2}}{2 R^{2}}$. It is therefore natural to choose constant sections $\Phi=\left\{\varphi^{m} \mid m \in \mathbb{Z}\right\}$ of the sheaf $\mathcal{S}$ over $\mathbb{R}^{+}$with $\mathcal{S}_{R}=\mathbb{H}_{R}$ by $\varphi_{R}^{m}:=|m\rangle_{R}$. Since the sections are constant with respect to the inner product on the Hilbert spaces $\mathbb{H}_{R}, R \in \mathbb{R}^{+}$, they are in particular compatible with the Hilbert space structure, which allows us to formally define a limiting Hilbert space

$$
\mathbb{H}_{(1)}^{\infty}:=\overline{\bigoplus_{m \in \mathbb{Z}} \operatorname{span}_{\mathbb{C}}\left\{|m\rangle_{\infty}\right\}} \text { with }|m\rangle_{\infty}:=\left\{|m\rangle_{R} \mid R \in \mathbb{R}^{+}\right\} .
$$

By (1.1.1), $\varphi^{m} \cdot \varphi^{m^{\prime}}=\varphi^{m+m^{\prime}}$ for all $m, m^{\prime} \in \mathbb{Z}$, so we are lead to set $A_{m}\left|m^{\prime}\right\rangle_{\infty}:=$ $\left|m+m^{\prime}\right\rangle_{\infty}$ and thus obtain a commutative algebra of bounded operators $\mathcal{A}_{(1)}^{\infty}:=$ $\left\langle A_{m} \mid m \in \mathbb{Z}\right\rangle$ on $\mathbb{H}_{(1)}^{\infty}$. The $H$-eigenvalues of all $|m\rangle_{R}$ converge to zero with the same speed as $R \rightarrow \infty$, hence we can naturally define $H_{(1)}^{\infty}|m\rangle_{\infty}:=\frac{m^{2}}{2}|m\rangle_{\infty}$ to obtain the commutative geometry $\left(\mathbb{H}_{(1)}^{\infty} \cong L^{2}\left(\mathbb{S}_{1}^{1}\right), H_{(1)}^{\infty}, \mathcal{A}_{(1)}^{\infty} \cong C^{\infty}\left(\mathbb{S}_{1}^{1}\right)\right)$ in the limit.

Mathematically, having $|m\rangle_{\infty}$ represent the sequence $\left\{|m\rangle_{R} \mid R \in \mathbb{R}^{+}\right\}$means that $\operatorname{span}_{\mathbb{C}}\left\{|m\rangle_{\infty}\right\}$ is the DIRECT LIMIT (see, e.g. Do ) of the $\left\{V_{m}^{R}, R \in \mathbb{R}^{+}\right\}$, where for $R, R^{\prime} \in \mathbb{R}^{+}$, we use $f_{R, R^{\prime}}: \mathbb{S}_{R^{\prime}}^{1} \rightarrow \mathbb{S}_{R}^{1}$ with $f_{R, R^{\prime}}(x):=x \cdot \frac{R}{R^{\prime}}$ to construct a DIRECT SYSTEM $\left(\mathbb{H}_{R}, f_{R, R^{\prime}}^{*}\right)$. Then, $\mathbb{H}_{(1)}^{\infty}$ is the DIRECT LIMIT of $\left(\mathbb{H}_{R}, f_{R, R^{\prime}}^{*}\right)$.

We have used the category $I_{1}$ with objects $\mathrm{Ob}\left(I_{1}\right) \cong \mathbb{R}^{+}$the circles of radii $R \in \mathbb{R}^{+}$and morphisms the diffeomorphisms between the circles. Note that there is precisely one diffeomorphism $f_{R, R^{\prime}}$ for every pair of $\operatorname{circles}^{8}\left(\mathbb{S}_{R^{\prime}}^{1}, \mathbb{S}_{R}^{1}\right)$.

\footnotetext{
8 We use oriented circles with base points to get rid of the translations and reflections.
} 
The limit $\left(\mathbb{H}_{(1)}^{\infty}, H_{(1)}^{\infty}, \mathcal{A}_{(1)}^{\infty}\right)$ is the inductive limit of the functor $F_{1}: I_{1} \rightarrow$ Vect which on objects maps $\mathbb{S}_{R}^{1} \mapsto C^{\infty}\left(\mathbb{S}_{R}^{1}\right)$, and on morphisms maps $f_{R, R^{\prime}} \mapsto f_{R, R^{\prime}}^{*}$.

Instead of $I_{1}$, there is another quite obvious category $I_{2}$ we could have chosen, namely with objects $\mathrm{Ob}\left(I_{2}\right)=\mathrm{Ob}\left(I_{1}\right) \cong \mathbb{R}^{+}$the circles of radii $R \in \mathbb{R}^{+}$and morphisms the isogenies (i.e. local isometries) between circles. That is, there exists a morphism $g_{R, R^{\prime}}: \mathbb{S}_{R^{\prime}}^{1} \rightarrow \mathbb{S}_{R}^{1}$ with $g_{R, R^{\prime}}(x):=x$ precisely if $\frac{R^{\prime}}{R} \in \mathbb{N}$. The inductive limit of the functor $F_{2}: I_{2} \rightarrow$ Vect which on objects maps $\mathbb{S}_{R}^{1} \mapsto$ $C^{\infty}\left(\mathbb{S}_{R}^{1}\right)$, and on morphisms maps $g_{R, R^{\prime}} \mapsto g_{R, R^{\prime}}^{*}$ is

$$
\begin{aligned}
\mathbb{H}_{\infty}^{(2)}:=\bigoplus_{\varrho \in \mathbb{R} / \mathbb{Q}} \operatorname{span}_{\mathbb{C}}\left\{|\varrho \bullet 0\rangle_{\infty}\right\} \oplus \bigoplus_{r \in \mathbb{R}^{*}} \operatorname{span}_{\mathbb{C}}\left\{|r\rangle_{\infty}\right\} \\
\text { with } \quad \forall \varrho \in \mathbb{R} / \mathbb{Q}:|\varrho \bullet 0\rangle_{\infty}:=\left\{|0\rangle_{N \varrho} \mid N \in \mathbb{Z}\right\}, \\
\forall r \in \mathbb{R}^{*}: \quad|r\rangle_{\infty}:=\left\{|n\rangle_{n / r} \mid n \in \mathbb{Z}\right\} .
\end{aligned}
$$

Here, $\mathbb{R} / \mathbb{Q}$ denotes classes of real numbers which are commensurable over $\mathbb{Q}$. We have $|\varrho \bullet 0\rangle_{\infty} \equiv 1$ for all $\varrho \in \mathbb{R} / \mathbb{Q}$, and for all $n \in \mathbb{Z}-\{0\}:|n\rangle_{n / r}: x \mapsto e^{i x r}$. Hence we naturally define $H_{(2)}^{\infty}|\varrho \bullet 0\rangle_{\infty}:=0$ for $\varrho \in \mathbb{R} / \mathbb{Q}$, and $H_{(2)}^{\infty}|r\rangle_{\infty}:=\frac{r^{2}}{2}|r\rangle_{\infty}$ for $r \in \mathbb{R}$. This again yields a degenerate limit, but we cannot rescale $H_{(2)}^{\infty}$ as before. Namely, to interpret the $|r\rangle_{\infty}$ in terms of sections $\varphi^{r}, r \in \mathbb{R}$ of $\mathcal{S}$ over $\mathbb{R}^{+}, \mathcal{S}_{R}=\mathbb{H}_{R}$ (where the label $r=0$ is replaced by $r=\varrho \bullet 0, \varrho \in \mathbb{R} / \mathbb{Q}$ ), we have to set $\varphi_{R}^{r}:=|r R\rangle_{R}$ iff $r R \in \mathbb{Z}\left(\varphi_{R}^{\varrho \bullet 0}:=|0\rangle_{R}\right.$ iff $R=\varrho$ in $\left.\mathbb{R} / \mathbb{Q}\right)$ and $\varphi_{R}^{r}:=0$, otherwise. To yield the $\varphi_{R}^{r}$ continuous, we need to introduce a discrete topology on $\mathbb{R}^{+}$. Then we can also naturally define a spectral triple $\left(\mathbb{H}_{(2)}^{\infty}, H_{(2)}^{\infty}, \mathcal{A}_{(2)}^{\infty}\right)$, with $A_{r} \in \mathcal{A}_{(2)}^{\infty}, r \in \mathbb{R}$, acting by $A_{r}\left|r^{\prime}\right\rangle_{\infty}=\left|r+r^{\prime}\right\rangle_{\infty}, A_{r}\left|r^{\prime} \bullet 0\right\rangle_{\infty}=|r\rangle_{\infty}$ iff $r$ and $r^{\prime}$ are commensurable over $\mathbb{Q}$, i.e. $r=r^{\prime}$ in $\mathbb{R} / \mathbb{Q}$, and $A_{r}\left|r^{\prime}\right\rangle_{\infty}=0$, $A_{r}\left|r^{\prime} \bullet 0\right\rangle_{\infty}=0$, otherwise. Similarly, for $\varrho \in \mathbb{R} / \mathbb{Q}$ we have $A_{\varrho \bullet \bullet} \in \mathcal{A}_{(2)}^{\infty}$ acting by $A_{\varrho \bullet 0}\left|r^{\prime}\right\rangle_{\infty}=\left|r^{\prime}\right\rangle_{\infty}, A_{\varrho \bullet 0}\left|r^{\prime} \bullet 0\right\rangle_{\infty}=\left|r^{\prime} \bullet 0\right\rangle_{\infty}$ iff $\varrho=r^{\prime}$ in $\mathbb{R} / \mathbb{Q}$ and $A_{\varrho \bullet \bullet}\left|r^{\prime}\right\rangle_{\infty}=0$, $A_{\varrho \bullet 0}\left|r^{\prime} \bullet 0\right\rangle_{\infty}=0$, otherwise. In other words, $A_{\varrho \bullet 0}$ acts as a projection, and $\left\{A_{\varrho \bullet 0} \mid \varrho \in \mathbb{R} / \mathbb{Q}\right\}$ defines a "partition of identity", $A_{0}:=\sum_{\varrho \in \mathbb{R} / \mathbb{Q}} A_{\varrho \bullet 0}$. This indeed gives a commutative geometry, namely $\mathbb{R}$ with the flat metric and an interesting topology.

Summarizing, Ex. 1.3.4 motivates the use of direct limits for the construction of limits of spectral pre-triples and CFTs. Moreover, as a word of caution, we have found two different limiting geometries for the spectral triples $\left(\mathbb{H}_{R}=L^{2}\left(\mathbb{S}_{R}^{1}\right)\right.$, $\left.H=\frac{1}{2} \Delta, \mathcal{A}_{R}=C^{\infty}\left(\mathbb{S}_{R}^{1}\right)\right)$ as $R \rightarrow \infty$, depending on the choice of the constant sections of $\mathcal{S}$ over $\mathbb{R}^{+}$with $\mathcal{S}_{R}=\mathbb{H}_{R}$. Both limits are natural in their own right. $\left(\mathbb{H}_{(1)}^{\infty}, H_{(1)}^{\infty}, \mathcal{A}_{(1)}^{\infty}\right)$ is motivated by the approach of $\left.[\mathrm{F}-\mathrm{G}] \mathrm{K}-\mathrm{S}\right]$, whereas $\left(\mathbb{H}_{(2)}^{\infty}, H_{(2)}^{\infty}\right.$, $\left.\mathcal{A}_{(2)}^{\infty}\right)$ corresponds to a decompactification of $\mathbb{S}_{R}^{1}$ as $R \rightarrow \infty$ equipped with a discrete topology. Similarly, the definition of limits for CFTs that we propose in Sect. 2 will incorporate some ambiguity.

\section{Remark 1.3.5}

We do not claim that direct limits yield the only sensible construction for limits of algebras or spectral triples as in Ex. 1.3.4. There, we have already performed a generalization from direct limits of ordered systems to direct limits of merely partially ordered systems. However, an ordered set $\left(\mathcal{A}_{i}, \bullet_{i},\langle., .\rangle_{i}\right)_{i \in I}$ of algebras 
with non-degenerate bilinear forms need not be a direct system at all in order to make sense of its "limit".

Since we mainly focus on the more natural direct limit construction, below, we do not give a formal definition of the more general one, here. The main idea, however, is to regard a vector space $\mathcal{A}$ as limit of the ordered set $\left(\mathcal{A}_{i}\right)_{i \in I}$ if for every $i \in I$ there is an epimorphism $f_{i}: \mathcal{A} \rightarrow \mathcal{A}_{i}$, such that for each $\varphi \in \mathcal{A}-\{0\}$ there exists an $N \in I$ with $f_{i}(\varphi) \neq 0$ for all $i>N$. If the respective limits, below, exist, then we can equip $\mathcal{A}$ with a limit bilinear form and algebra structure by setting

$$
\langle\varphi, \chi\rangle:=\lim _{i}\left\langle f_{i}(\varphi), f_{i}(\chi)\right\rangle_{i}, \quad\langle\psi, \varphi \bullet \chi\rangle:=\lim _{i}\left\langle f_{i}(\psi), f_{i}(\varphi) \bullet_{i} f_{i}(\chi)\right\rangle_{i} .
$$

Note that this only defines an algebra structure on $\mathcal{A}$ if $\langle\cdot, \cdot\rangle$ is non-degenerate.

As an example let us discuss the limit of the algebras $C^{\infty}\left(\mathbb{S}_{R}^{1}\right)$ of Ex. 1.3.4 equipped with the Hermitean form

$$
\langle\varphi, \chi\rangle_{R}=\frac{1}{2 \pi R} \int_{0}^{2 \pi R} \overline{\varphi(x)} \chi(x) d x .
$$

The radii $R \in \mathbb{R}^{+}$of the circles $\mathbb{S}_{R}^{1}$ constitute the ordered index set $I$. As limit space $\mathcal{A}$ we choose the space $C_{c}^{\infty}(\mathbb{R})$ of compactly supported smooth functions on $\mathbb{R}$. Then, we define

$$
\begin{aligned}
f_{R}: C_{c}^{\infty}(\mathbb{R}) & \longrightarrow C^{\infty}\left(\mathbb{S}_{R}^{1}\right) \\
\varphi(x) & \longmapsto f_{R}(\varphi)(y):=\frac{1}{\sqrt{R}} \sum_{m \in \mathbb{Z}} \varphi\left(\frac{m}{R}\right) e^{i R y / m},
\end{aligned}
$$

which is a discrete version of a Fourier transform. Indeed, $\left(C^{\infty}\left(\mathbb{S}_{R}^{1}\right), f_{R}\right)_{R \in \mathbb{R}^{+}}$ fulfills all the conditions mentioned above, and the limit algebra structure on $\mathcal{A}=C_{c}^{\infty}(\mathbb{R})$, corresponding to the ordinary product of the Fourier transformed functions, is the convolution product

$$
\varphi \bullet \chi(x)=\int_{\mathbb{R}} \varphi(x-y) \chi(y) d y .
$$

This construction can be extended to a limit of spectral triples as in Ex. 1.3.4 and the limit geometry is $\mathbb{R}$ with the standard topology.

\section{Limits of conformal field theories: Definitions}

This section gives our main definitions and is the technical heart of the paper. As explained above, our construction is motivated by the ideas of [F-G] K-S]. The guiding example is that of the circle theories discussed in Exs.1.1.1 1.2.2 1.3.4 or more generally the toroidal CFTs discussed in Sect. 3.1 since these models as well as their large volume limits are well understood. Further motivation arises from the observation that the family of unitary Virasoro minimal models $\mathcal{M}(m, m+1), m \in \mathbb{N}-\{0,1\}$, can be treated by our techniques, too, as detailed in Sect. 4

Sect. [2.1] is devoted to the definition of sequences of CFTs and their limits; we propose a list of conditions which ensure that the limit possesses enough structure in order to realize some of the ideas of [F-G] K-S. In Sect. 2.2 we explain how our limits can give rise to geometric interpretations. 
2.1. Sequences of CFTs and their limits. In Ex. 1.3.4 we have given a motivation for our general approach to limiting processes for CFTs, which uses direct systems and direct limits ${ }^{9}$. We recall the basic definitions below but refer the reader to the literature for a more detailed exposition, see e.g. Do. We start by defining sequences of CFTs:

Definition 2.1.1

Let $\left(\mathcal{C}^{i}\right)_{i \in \mathbb{N}}=\left(\mathcal{H}^{i}, *^{i}, \Omega^{i}, T^{i}, \bar{T}^{i}, C^{i}\right)_{i \in \mathbb{N}}$ denote a family of CFTs with left and right central charges $c_{i}$. Given vector space homomorphisms $f_{i}^{j}$ such that

$$
\left.\begin{array}{l}
\forall i, j \in \mathbb{N}, i \leq j: f_{i}^{j}: \mathcal{H}^{i} \longrightarrow \mathcal{H}^{j}, \quad \text { and } \\
f_{i}^{j}\left(\Omega^{i}\right)=\Omega^{j}, \\
f_{i}^{j}\left(T^{i}\right)=T^{j}, \\
f_{i}^{j}\left(\bar{T}^{i}\right)=\bar{T}^{j},
\end{array}\right\} \text { and } \forall i, j, k \in \mathbb{N}, i \leq j \leq k:\left\{\begin{array}{r}
f_{i}^{i}=i d_{\mathcal{H}^{i}}, \\
f_{j}^{k} f_{i}^{j}=f_{i}^{k}, \\
*^{j} f_{i}^{j}=f_{i}^{j} *^{i},
\end{array}\right.
$$

we call $\left(\mathcal{C}^{i}, f_{i}^{j}\right)$ a SEQUENCE OF CONFORMAL FIELD THEORIES.

Note that we do not demand any further CFT-structure to be preserved by the morphisms $f_{i}^{j}$, which therefore are not morphisms of CFTs. Hence a sequence of CFTs cannot be regarded as a direct system of CFTs. However, 2.1.1) by definition gives a direct system of vector spaces $\left(\mathcal{H}^{i}, f_{i}^{j}\right)$. It allows us to define a direct limit vector space Do

$$
\mathcal{K}^{\infty}:=\lim _{\longrightarrow} \mathcal{H}^{k} \simeq\left[\bigoplus_{k \in \mathbb{N}} \mathcal{H}^{k}\right] / \operatorname{span}_{\mathbb{C}}\left\{\varphi^{i}-f_{i}^{j}\left(\varphi^{i}\right) \mid i, j \in \mathbb{N}, i \leq j, \varphi^{i} \in \mathcal{H}^{i}\right\},
$$

where by abuse of notation for $i \in \mathbb{N}$ we have omitted the inclusion homomorphisms $\imath^{i}: \mathcal{H}^{i} \hookrightarrow \bigoplus_{k \in \mathbb{N}} \mathcal{H}^{k}$. The above definition of $\mathcal{K}^{\infty}$ means that for each $\varphi \in \mathcal{K}^{\infty}$ there exist $k \in \mathbb{N}$ and $\varphi^{k} \in \mathcal{H}^{k}$ such that $\varphi$ is represented by $\varphi^{k}$, i.e. $\varphi=\left[\varphi^{k}\right]=\left[f_{k}^{l}\left(\varphi^{k}\right)\right]$ for all $l \geq k$. In the following, $\varphi^{k}$ will always denote a representative of this form for $\varphi \in \mathcal{K}^{\infty}$. By

$$
\forall i \in \mathbb{N}: \quad f_{i}^{\infty}: \quad \mathcal{H}^{i} \stackrel{i^{i}}{\longrightarrow} \bigoplus_{k \in \mathbb{N}} \mathcal{H}^{k} \stackrel{\text { proj }}{\longrightarrow} \mathcal{K}^{\infty}
$$

we denote the homomorphisms given by the composition of inclusion and projection, with $f_{j}^{\infty} \circ f_{i}^{j}=f_{i}^{\infty}$ for $i, j \in \mathbb{N}, i \leq j$. With the above notations, $\varphi=f_{k}^{\infty}\left(\varphi^{k}\right) \in \mathcal{K}^{\infty}$.

Similarly, for $i, j \in \mathbb{N}, i \leq j$ and $\psi \in \mathcal{H}^{i}$ we define $\left(f_{i}^{j}\right)^{*}\left(\psi^{*}\right):=\left(f_{i}^{j}(\psi)\right)^{*}$. This gives a direct system $\left(\left(\check{\mathcal{H}}^{*}\right)^{i},\left(f_{i}^{j}\right)^{*}\right)$. Its direct limit is denoted $\left(\check{\mathcal{K}}^{*}\right)^{\infty}$, and we have projections $\left(f_{i}^{*}\right)^{\infty}=\left(f_{i}^{\infty}\right)^{*}$ as above.

By (2.1.1), the limits $\mathcal{K}^{\infty}$ and $\left(\check{\mathcal{K}}^{*}\right)^{\infty}$ possess special elements $\Omega, T, \bar{T}$ and $\Omega^{*}$, and an involution $*$. However, the definition of CFT-like structures on the limit vector space $\mathcal{K}^{\infty}$ requires some more conditions on a sequence of CFTs, which we shall discuss now. In particular, we need a notion of convergence.

\footnotetext{
9 Although not spelled out in this language, in [M-S2 §6] a notion of classical limits of CFTs in terms of direct limits was introduced.
} 
In the following, let $\left(\mathcal{C}^{i}, f_{i}^{j}\right)$ denote a sequence of CFTs.

\section{Condition 1}

The OPE-coefficients $C^{i}$ of $\mathcal{C}^{i}$ CONVERGe with RESPECt to the $f_{i}^{j}$, i.e.

$$
\begin{aligned}
& \forall \varphi \in \mathcal{H}^{i}, \chi \in \mathcal{H}^{j}, \psi \in \mathcal{H}^{k}: \\
& \quad C^{m}\left(\left(f_{k}^{m}(\psi)\right)^{*}, f_{i}^{m}(\varphi), f_{j}^{m}(\chi)\right) \stackrel{m \rightarrow \infty}{\longrightarrow} C\left(\left(f_{k}^{\infty}\right)^{*}\left(\psi^{*}\right), f_{i}^{\infty}(\varphi), f_{j}^{\infty}(\chi)\right) \in \mathbb{C} .
\end{aligned}
$$

The limits $C$ of the OPE-coefficients only depend on elements of the direct limits $\mathcal{K}^{\infty},\left(\check{\mathcal{K}}^{*}\right)^{\infty}$ and are trilinear. Thus a sequence of CFTs $\left(\mathcal{C}^{i}, f_{i}^{j}\right)$ fulfilling Cond. 1 gives rise to a trilinear function

$$
C^{\infty}:\left(\check{\mathcal{K}}^{\infty}\right)^{*} \otimes \mathcal{K}^{\infty} \otimes \mathcal{K}^{\infty} \longrightarrow \mathbb{C}
$$

$C^{i}, *^{i}$, and the map $\mathcal{H}^{i} \rightarrow\left(\check{\mathcal{H}}^{i}\right)^{*}, \psi \mapsto \psi^{*}$ with (1.2.3), (1.2.4) determine the Hermitean structure of $\mathcal{H}^{i}$. Since the homomorphisms $f_{i}^{j}$ are compatible with this structure, if Cond. 1 holds, then the vector space $\mathcal{K}^{\infty}$ inherits a limiting bilinear form $\langle\cdot \mid \cdot\rangle_{\infty}=C^{\infty}\left(*^{*}, \Omega, \cdot\right)$, which may be degenerate, though. Define $\mathcal{N}^{\infty} \subset \mathcal{K}^{\infty}$ to be the space of NULL VECTORS of $\langle\cdot \mid \cdot\rangle_{\infty}$ in $\mathcal{K}^{\infty}$, i.e.

$$
\mathcal{N}^{\infty}:=\left\{\nu \in \mathcal{K}^{\infty} \mid C^{\infty}\left(\nu^{*}, \Omega, \nu\right)=0\right\} .
$$

Since the Cauchy-Schwarz inequality is valid for all $\langle\cdot \mid \cdot\rangle_{i}=C^{i}\left(\cdot^{*}, \Omega^{i}, \cdot\right)$, Cond. [1 implies that $C^{\infty}\left(.^{*}, \Omega, \cdot\right)$ defines a non-degenerate bilinear form on

$$
\mathcal{H}^{\infty}:=\mathcal{K}^{\infty} / \mathcal{N}^{\infty} \quad \text { with } \quad \pi^{\infty}: \mathcal{K}^{\infty} \longrightarrow \mathcal{H}^{\infty} \text { the projection. }
$$

In the following, we will frequently use elements $\varphi \in \mathcal{K}^{\infty}$ to represent a class in $\mathcal{H}^{\infty}$ and by abuse of notation write $\varphi \in \mathcal{H}^{\infty}$. Note that $\pi^{\infty}$ is compatible with $C^{\infty}$ only if the following condition holds:

\section{Condition 2}

All OPE-constants involving null vectors $\nu \in \mathcal{N}^{\infty}$ as in (2.1.2) vanish in the limit, i.e. the following conditions hold:

$$
\begin{array}{ll}
\forall \nu \in \mathcal{N}^{\infty}, \forall \varphi, \chi \in \mathcal{K}^{\infty}: & C^{\infty}\left(\chi^{*}, \nu, \varphi\right)=0 \\
& C^{\infty}\left(\chi^{*}, \varphi, \nu\right)=0=C^{\infty}\left(\nu^{*}, \varphi, \chi\right) .
\end{array}
$$

By (A.10, the latter two conditions are equivalent.

Cond. [2] implies that $C^{\infty}$ descends to a well-defined map

$$
C^{\infty}:\left(\check{\mathcal{H}}^{\infty}\right)^{*} \otimes \mathcal{H}^{\infty} \otimes \mathcal{H}^{\infty} \longrightarrow \mathbb{C} .
$$

Though short and elegant, Cond. 2] seems not to be very convenient to check in our applications. See Rem. 2.1.5 iil for a simplification and note that in our Def. 2.1 .3 we avoid this difficulty.

In order to recover a CFT-like structure in the limit, we will introduce a direct limit of the decomposition (1.2.1) on $\mathcal{H}^{\infty}$. To this end, we will need 


\section{Condition 3}

There are decompositions of the vector spaces $\mathcal{H}^{i}$ into common $L_{0^{-}}^{i}$ and $\bar{L}_{0}^{i}$-eigenspaces, which are preserved by the $f_{i}^{j}$, i.e.

$$
\begin{aligned}
\mathcal{H}^{i}=\bigoplus_{\alpha \in \mathcal{I}_{i}} \mathcal{H}_{\alpha}^{i},\left.\quad L_{0}^{i}\right|_{\mathcal{H}_{\alpha}^{i}}=h_{\alpha}^{i} \operatorname{id}_{\mathcal{H}_{\alpha}^{i}},\left.\quad \bar{L}_{0}^{i}\right|_{\mathcal{H}_{\alpha}^{i}}=\bar{h}_{\alpha}^{i} \operatorname{id}_{\mathcal{H}_{\alpha}^{i}}, \\
\quad \forall i, j \in \mathbb{N}, \forall \alpha \in \mathcal{I}_{i} \exists \beta \in \mathcal{I}_{j}: f_{i}^{j}\left(\mathcal{H}_{\alpha}^{i}\right) \subset \mathcal{H}_{\beta}^{j} ; \quad f_{i}^{j}(\alpha):=\beta .
\end{aligned}
$$

In fact, the induced maps $f_{i}^{j}: \mathcal{I}_{i} \longrightarrow \mathcal{I}_{j}$ defined by Cond. 3 constitute a direct system on the index sets, whose direct limit will be called $\mathcal{I}_{\infty}:=\underset{\longrightarrow}{\longrightarrow} \mathcal{I}_{i}$. The preservation of the decompositions by the $f_{i}^{j}$ guarantees the existence of a decomposition

$$
\mathcal{K}^{\infty}=\bigoplus_{\alpha \in \mathcal{I}_{\infty}} \mathcal{K}_{\alpha}^{\infty}
$$

Cond. 3even guarantees that if Cond. 11 is satisfied as well, then (2.1.4 imposes an analogous decomposition of $\mathcal{N}^{\infty}$ and therefore results in

$$
\mathcal{H}^{\infty}=\bigoplus_{\alpha \in \mathcal{I}_{\infty}} \mathcal{H}_{\alpha}^{\infty} .
$$

For $\varphi \in \mathcal{H}_{\alpha}^{\infty}$ with $\alpha=\left[\alpha^{k}\right], \varphi \neq 0$,

$$
h_{\alpha^{k}}=h_{\varphi^{k}}:=\frac{C^{k}\left(\left(\varphi^{k}\right)^{*}, T^{k}, \varphi^{k}\right)}{C^{k}\left(\left(\varphi^{k}\right)^{*}, \Omega^{k}, \varphi^{k}\right)}, \quad \bar{h}_{\alpha^{k}}=\bar{h}_{\varphi^{k}}:=\frac{C^{k}\left(\left(\varphi^{k}\right)^{*}, \bar{T}^{k}, \varphi^{k}\right)}{C^{k}\left(\left(\varphi^{k}\right)^{*}, \Omega^{k}, \varphi^{k}\right)}
$$

give the $\left(L_{0}^{k}, \bar{L}_{0}^{k}\right)$ eigenvalues of $\varphi^{k}$. By Conds. 1 and 2 all limits

$$
h_{\alpha}:=\lim _{k \rightarrow \infty} h_{\alpha^{k}}^{k}, \quad \bar{h}_{\alpha}:=\lim _{k \rightarrow \infty} \bar{h}_{\alpha^{k}}^{k}
$$

exist. Therefore, we can define the following operators on $\mathcal{H}^{\infty}$ :

$$
\left.L_{0}\right|_{\mathcal{H}_{\alpha}^{\infty}}:=h_{\alpha} \operatorname{id}_{\mathcal{H}_{\alpha}^{\infty}},\left.\quad \bar{L}_{0}\right|_{\mathcal{H}_{\alpha}^{\infty}}:=\bar{h}_{\alpha} \mathrm{id}_{\mathcal{H}_{\alpha}^{\infty}} .
$$

These give rise to a coarser decomposition of $\mathcal{H}^{\infty}$ than 2.1.5 into $\left(L_{0}, \bar{L}_{0}\right)$ eigenspaces,

$$
\mathcal{H}^{\infty}=\bigoplus_{\substack{h, h \in \mathbb{R}, h-\bar{h} \in \mathbb{Z}}} \mathcal{H}_{h, \bar{h}}^{\infty}
$$

In particular, as opposed to a well-defined CFT, it is not guaranteed that all $\mathcal{H}_{h, \bar{h}}^{\infty}$ are finite dimensional. Indeed, the $\mathcal{H}_{h, \bar{h}}^{\infty}$ will be infinite dimensional for some of the examples studied in Sects. 3 and 4 In order to nevertheless allow a definition of modes analogously to (A.13), we will therefore need

\section{Condition 4}

For all $\alpha \in \mathcal{I}_{\infty}$ and all $\varphi \in \mathcal{H}^{\infty}, \chi \in \mathcal{H}_{\alpha}^{\infty}$,

$$
\mathcal{I}_{\mu, \bar{\mu}}(\varphi, \chi):=\left\{\beta \in \mathcal{I}_{\infty} \mid \mathcal{H}_{\beta}^{\infty} \subset \mathcal{H}_{h_{\alpha}+\mu, \bar{h}_{\alpha}+\bar{\mu}}^{\infty}, \quad \exists \psi \in \mathcal{H}_{\beta}^{\infty}: C^{\infty}\left(\psi^{*}, \varphi, \chi\right) \neq 0\right\}
$$


is finite, such that

$$
V_{\mu, \bar{\mu}}(\varphi, \chi):=\bigoplus_{\beta \in \mathcal{I}_{\mu, \bar{\mu}}(\varphi, \chi)} \mathcal{H}_{\beta}^{\infty}
$$

is finite dimensional.

Cond. 4 can also be derived from a version of uniform convergence on the $C^{i}$ which we discuss in Rem. 2.1.5

To summarize, a sequence of CFTs which obeys Conds. 10 4 gives rise to a limit vector space $\mathcal{H}^{\infty}$ with non-degenerate bilinear form $\langle\cdot \mid \cdot\rangle_{\infty}=C^{\infty}\left(\cdot{ }^{*}, \Omega, \cdot\right)$ and an OPE-like structure, which assigns modes to each vector in this vector space analogously to A.13:

$$
\begin{aligned}
& \forall \varphi \in \mathcal{H}^{\infty}, \forall \mu, \bar{\mu}, \in \mathbb{R}, \forall \alpha \in \mathcal{I}_{\infty}, \forall \chi \in \mathcal{H}_{h_{\alpha}, \bar{h}_{\alpha}}: \\
& \varphi_{\mu, \bar{\mu}} \chi \in V_{\mu, \bar{\mu}}(\varphi, \chi) \text { s.th. } \forall \psi \in V_{\mu, \bar{\mu}}(\varphi, \chi): \psi^{*}\left(\varphi_{\mu, \bar{\mu}} \chi\right)=C^{\infty}\left(\psi^{*}, \varphi, \chi\right) .
\end{aligned}
$$

Recall that for a well-defined CFT, the modes of specific subsectors form closed algebras, like the holomorphic and antiholomorphic W-algebras. However, we need additional conditions which ensure that this algebra structure is preserved in (2.1.6). We first specify

\section{Definition 2.1.2}

For a sequence $\left(\mathcal{C}^{i}, f_{i}^{j}\right)$ of CFTs, let $\widetilde{\mathcal{W}}^{i}$ denote a sequence of finite subsets of $\operatorname{ker} \bar{L}_{0}^{i} \oplus \operatorname{ker} L_{0}^{i}$ with $T^{i}, \bar{T}^{i} \in \widetilde{\mathcal{W}}^{i}$ and $f_{i}^{j}\left(\widetilde{\mathcal{W}}^{i}\right)=\widetilde{\mathcal{W}}^{j}$, which generate subalgebras

$$
\mathcal{W}^{i}:=\left\langle\varphi_{n, 0}, \varphi_{0, n} \mid \varphi \in \operatorname{span}_{\mathbb{C}} \widetilde{\mathcal{W}}^{i}, n \in \mathbb{Z}\right\rangle
$$

of the holomorphic and antiholomorphic $W$-algebras. Assume that the $\mathcal{W}^{i}$ are all of the same type, i.e. they differ only by their structure constants with respect to the elements of $\widetilde{\mathcal{W}}^{i}$. Then the family $\mathcal{W}^{i} \supset \mathcal{V}_{i_{i}} \oplus \overline{\mathcal{V i r}}_{c_{i}}$ is called the STABLE $\mathrm{W}$-ALGEBRA, and the elements of $\widetilde{\mathcal{W}}^{i}$ are called $\mathrm{W}$-STATES.

By definition, the Virasoro algebra is stable in every sequence of CFTs, and we denote

$$
\widetilde{\mathcal{W}}^{\infty}:=\pi^{\infty} f_{i}^{\infty}\left(\widetilde{\mathcal{W}}^{i}\right)
$$

To guarantee that (2.1.6) induces the action of a W-algebra on $\mathcal{H}^{\infty}$, the stable $\mathrm{W}$-algebras have to obey the following two conditions:

\section{Condition 5}

The $f_{i}^{j}$ preserve the primaries of the $\mathcal{W}^{i}$, which never become null:

$$
\forall i, j \in \mathbb{N}: \quad f_{i}^{j}\left(\left(\mathcal{H}^{i}\right)^{\mathcal{W}^{i}}\right) \subset\left(\mathcal{H}^{j}\right)^{\mathcal{W}^{j}}, \quad \text { and } \quad f_{i}^{\infty}\left(\left(\mathcal{H}^{i}\right)^{\mathcal{W}^{i}}-\{0\}\right) \cap \mathcal{N}^{\infty}=\emptyset .
$$

\section{Condition 6}

For every holomorphic $W$-state $w$ and $\chi \in \mathcal{K}^{\infty}, n \in \mathbb{Z}$, the sequence $w_{n, 0}^{i} \chi^{i}$ CONVERGES WEAKLY TO $w_{n, 0} \chi$ as defined by (2.1.6), that is:

$$
\begin{aligned}
& \forall \chi, \psi \in \mathcal{K}^{\infty}, \forall w, \widetilde{w} \in \widetilde{\mathcal{W}}^{\infty}, \forall n \in \mathbb{Z}: \\
& C^{\infty}\left(\psi^{*}, \widetilde{w}, w_{n, 0} \chi\right)=\lim _{i \rightarrow \infty} C^{i}\left(\left(\psi^{i}\right)^{*}, \widetilde{w}^{i}, w_{n, 0}^{i} \chi^{i}\right),
\end{aligned}
$$


and analogously for antiholomorphic W-states $w$.

Indeed, a sequence of CFTs with stable $\mathrm{W}$-algebras $\mathcal{W}^{i}$ which obeys Conds. 16 features a $\mathrm{W}$-algebra action of $\mathcal{W}^{\infty}$ on $\mathcal{H}^{\infty}$, generated by the modes of all $\mathrm{W}$ states in $\widetilde{\mathcal{W}}^{\infty}$, and with structure constants obtained as limits of the structure constants of the $\mathcal{W}^{i}$. The stable $\mathrm{W}$-algebras $\mathcal{W}^{i}$ are non-trivial by definition, since at least $\mathcal{V}_{c_{i}}$ and $\overline{\mathcal{V i r}}_{c_{i}}$ are stable. Hence, for example

$$
c:=2 C^{\infty}\left(\Omega^{*}, T, L_{2} \Omega\right) \stackrel{\text { 2.1.7 }}{=} 2 \lim _{i \rightarrow \infty} C^{i}\left(\left(\Omega^{i}\right)^{*}, T^{i}, L_{2}^{i} \Omega^{i}\right)=\lim _{i \rightarrow \infty} c_{i}
$$

gives the central charge of the limiting Virasoro algebras $\operatorname{Vir}_{c}, \overline{\mathcal{V i r}}_{c} \subset \mathcal{W}^{\infty}$. Analogously, as expected, for $\varphi \in \mathcal{H}_{h, \bar{h}}^{\infty}, \varphi \neq 0$, we have

$$
h=\frac{1}{2} C^{\infty}\left(\varphi^{*}, T, L_{1} \varphi\right) /|\varphi|^{2}, \quad \bar{h}=\frac{1}{2} C^{\infty}\left(\varphi^{*}, \bar{T}, \bar{L}_{1} \varphi\right) /|\varphi|^{2} .
$$

Finally, in order to introduce a limit of the truncated OPE (1.2.6) for all states that are relevant for our geometric interpretations in Sect. 2.2 we will need

\section{Condition 7}

For all $\alpha, \beta \in \mathcal{I}_{\infty}$ and all $\varphi \in \mathcal{H}_{\alpha}^{\infty}, \chi \in \mathcal{H}_{\beta}^{\infty}$, let

$$
I(\varphi, \chi):=\left\{(\mu, \bar{\mu}) \in \mathbb{R}^{2} \mid \exists \psi \in f_{i}^{\infty}\left(\left(\mathcal{H}^{i}\right)^{\mathcal{W}^{i}}\right): \psi^{*}\left(\varphi_{\mu, \bar{\mu}} \chi\right) \neq 0\right\},
$$

as in (1.2.6). If $\mathcal{H}_{\alpha}^{\infty} \subset \mathcal{H}_{0,0}^{\infty}$, then $|I(\varphi, \chi)|<\infty$.

We would like to point out that Cond. 7 is required to ensure the finiteness condition used in the definition of the truncated OPE $⿴$ (1.2.6) in the limit. As mentioned there, this finiteness condition however seems to be very restrictive and can probably be replaced by appropriate normalizability of the vectors $\sum_{(\mu, \bar{\mu}) \in I_{\mathcal{W}}(\varphi, \chi)} \varphi_{\mu, \bar{\mu}} \chi$ for $\varphi, \chi \in \mathcal{H}^{\mathcal{W}}$. Then Cond. [7 would be dispensable.

We are now ready to give a definition of convergence for CFTs which we find natural: Any sequence of CFTs obeying Conds. 1 - 7 will carry an OPE-like structure on $\mathcal{H}^{\infty}$ with an action of the limiting $\mathrm{W}$-algebra $\mathcal{W}^{\infty}$ on $\mathcal{H}^{\infty}$ which is compatible with the mode construction. However, so far, we have used an "algebraic" approach to CFTs as described at the beginning of Sect. 1.2 and in App. A This approach has the drawback that the "analytic" structure of CFT $n$-point functions, also explained in App. $\mathrm{A}$ is encoded in a rather complicated way. It makes some of the above conditions quite intricate, but does not ensure the existence of $n$-point functions in the limit. On the other hand, some of these conditions follow from convergence of four-point functions on the sphere. In fact, Conds. 1- 7 alone turn out to be problematic in view of our aim to find geometric interpretations of the limit, similar to Def. 1.3.2. We have not succeeded to derive commutativity of the relevant algebra $\mathcal{A}^{\infty}$ from them, see Prop. 2.2.4 Therefore, we formulate a notion of convergence of sequences of CFTs which poses stronger conditions by incorporating the "analytic" structure of CFTs:

Definition 2.1.3

Let $\left(\mathcal{C}^{i}, f_{i}^{j}\right)$ denote a sequence of CFTs with stable $W$-algebras $\mathcal{W}^{i}$, whose fourpoint functions on $\mathbb{P}^{1}$ converge with respect to the $f_{i}^{j}$ as real analytic functions 
outside the partial diagonals, with the standard behaviour near the singularities (see App. A). In other words, for all $i \in \mathbb{N}, \varphi, \chi, \psi, \omega \in \mathcal{H}^{i}$,

$$
(z, \bar{z}) \longmapsto \lim _{j \rightarrow \infty}\left\langle f_{i}^{j}(\psi)\left|f_{i}^{j}(\varphi)(1,1) f_{i}^{j}(\chi)(z, \bar{z})\right| f_{i}^{j}(\omega)\right\rangle_{j}
$$

exists as real analytic function of $z, \bar{z} \in \mathbb{C} \backslash\{0,1\} \cong \mathbb{P}^{1} \backslash\{0,1, \infty\}$ with expansions (A.4) around the points $0,1, \infty$. If the sequence moreover fulfills Conds. 3 - 7 then it is called FULLY CONVERGENT.

As a word of caution we remark that in general, the limits of four-point functions do not descend to well-defined objects on $\left(\mathcal{H}^{\infty}\right)^{\otimes 4}$.

\section{Proposition 2.1.4}

Let $\left(\mathcal{C}^{i}, f_{i}^{j}\right)$ denote a fully convergent sequence of CFTs with stable $W$-algebras $\mathcal{W}^{i}$. Then this sequence obeys Conds. [- 1 .

Proof:

We need to show that Conds. 1 1 and 2 follow from the convergence of four-point functions on the sphere and Conds. 3- -7

Indeed, Cond. 1 is an immediate consequence, since for $\varphi, \chi, \psi \in \mathcal{H}^{i}$ :

$$
\left\langle\psi\left|\varphi(1,1) \Omega^{i}(z, \bar{z})\right| \chi\right\rangle_{i} \equiv\langle\psi|\varphi(1,1)| \chi\rangle_{i} \stackrel{\text { A.8 }}{=} C^{i}\left(\psi^{*}, \varphi, \chi\right),
$$

and $f_{i}^{j}\left(\Omega^{i}\right)=\Omega^{j}$.

To see that Cond. 2] is satisfied, first assume that there are vectors $\nu \in$ $\mathcal{N}_{a, \bar{a}}^{\infty}, \varphi \in \mathcal{K}_{b, \bar{b}}^{\infty}, \chi \in \mathcal{K}_{c, \bar{c}}^{\infty}$ such that $C^{\infty}\left(\nu^{*}, \varphi, \chi\right) \neq 0$, and choose a sequence $\left\{\widetilde{\psi}_{j}^{i}\right\}_{j}$ of orthogonal bases of the $\mathcal{H}^{i}, \widetilde{\psi}_{j}^{i} \in \mathcal{H}_{h_{j}^{i}}^{i}, \bar{h}_{j}^{i}$, which converges weakly to an orthogonal basis of $\mathcal{K}^{\infty}$. Using (A.10), we can expand the following four-point function around $z=0$ as in (A.9):

$$
\left\langle\chi^{i}\left|\left(\varphi^{i}\right)^{\dagger}(1,1) \varphi^{i}(z, \bar{z})\right| \chi^{i}\right\rangle_{i}=\sum_{j}\left|\frac{C^{i}\left(\left(\widetilde{\psi}_{j}^{i}\right)^{*}, \varphi^{i}, \chi^{i}\right)}{\left|\widetilde{\psi}_{j}^{i}\right|^{i}}\right|^{2} z^{h_{j}^{i}-b^{i}-c^{i}} \bar{z}^{\bar{h}_{j}^{i}-\bar{b}^{i}-\bar{c}^{i}}
$$

In particular, we can choose $\widetilde{\psi}_{1}^{i}:=\nu^{i}$ and obtain a contradiction to the convergence of four-point functions. By A.10), this also contradicts the existence of $\nu, \varphi, \chi$ as above with $C^{\infty}\left(\chi^{*}, \varphi, \nu\right) \neq 0$.

Finally, A.12 shows that $C^{\infty}\left(\psi^{*}, \nu, \chi\right)=0$ follows for all $\nu \in \mathcal{N}^{\infty}, \chi \in$ $\mathcal{K}^{\infty}$, and primary $\psi \in \mathcal{K}^{\infty}$. But all three-point functions on the sphere which involve descendants can be obtained from those involving the corresponding primaries by application of differential operators. Hence the convergence of fourpoint functions on the sphere together with Conds. [5] ensure that this suffices to prove the claim.

Although in the limit of a sequence of CFTs we get well-defined OPE-coefficients by construction, we cannot expect convergence of all correlation functions, as e.g. torus partition functions. This means that the limit of a converging sequence of CFTs is not a CFT, in general. It may be possible to pose more restrictive conditions on the notion of convergence in order to ensure the limit to be described by a well-defined CFT. However, we are interested in certain degeneration phenomena, which for example occur in the large volume limits mentioned in Exs. 
1.2.2 1.3.4 These limits do not have a well-defined torus partition function. So from our viewpoint it is not even desirable to have a CFT as limit of a converging sequence, in general. We wish to emphasize that a limit in the above sense severely depends on the choice of the homomorphisms $f_{i}^{j}$ in our sequence $\left(\mathcal{C}^{i}, f_{i}^{j}\right)$ of CFTs. This is analogous to the ambiguity described in Ex. 1.3.4

\section{Remark 2.1.5}

i. Since for $\psi \in \mathcal{H}_{h, h}^{i}$ in a convergent sequence of CFTs $\left\langle\Omega^{i}\left|\Omega^{i}(1,1) \psi(z, \bar{z})\right| \psi\right\rangle_{i}$ $=C^{i}\left(\left(\Omega^{i}\right)^{*}, \psi, \psi\right) z^{-2 h} \bar{z}^{-2 \bar{h}}$, the convergence of the $\left(L_{0}, \bar{L}_{0}\right)$ eigenvalues for states with non-vanishing norm follows independently from the arguments given after Cond. 3

ii. The crucial step in the proof of Prop. 2.1.4 is the observation that each coefficient in (2.1.9) remains bounded in the limit. The latter is equivalent to the following condition:

$$
\begin{aligned}
& \text { For } \chi, \psi \in \mathcal{K}^{\infty} \text { set } \mu^{i}:=h_{\psi^{i}}-h_{\chi^{i}}, \bar{\mu}^{i}:=\bar{h}_{\psi^{i}}-\bar{h}_{\chi^{i}} ; \\
& \text { then } \forall \varphi \in \mathcal{K}^{\infty}: \quad \mid \varphi_{\mu^{i}, \bar{\mu}^{i}}^{i} \chi^{i} \quad \text { is bounded as } i \rightarrow \infty .
\end{aligned}
$$

Hence 2.1.10 implies Cond. 2 and is equivalent to the convergence of fourpoint functions $\left\langle\chi^{i}\left|\left(\varphi^{i}\right)^{\dagger}(1,1) \varphi^{i}(z, \bar{z})\right| \chi^{i}\right\rangle_{i}$.

iii. In general, null vectors of a representation of the Virasoro algebras $\mathcal{V i r}_{c}, \overline{\mathcal{V i r}}_{c}$ are defined to be states that descend from a lowest weight vector but vanish under the action of each $L_{n}, \bar{L}_{n}$ with $n<0$. Although our definition (2.1.2) of null vectors is different, a fully convergent sequence of CFTs has stable Virasoro algebras and allows us to define the action of $\mathcal{V i r}_{c}, \overline{\mathcal{V i r}}_{c}$ on $\mathcal{H}^{\infty}$ such that null vectors in this conventional sense are not present, either.

iv. Our definition of a fully convergent sequence of CFTs with stable W-algebras $\mathcal{W}^{i}$ simplifies greatly if $\mathcal{N}^{\infty}$ as in (2.1.2) reduces to $\{0\}$. Then Cond. 2] is void. Moreover, Cond. [6 follows from the convergence of four-point functions, since the limiting four-point functions are well-defined on $\mathcal{H}^{\infty}$ and the factorization properties A.9, A.17 - A.18 of four-point functions remain valid in the limit. As in ordinary CFTs, this also implies associativity of the OPE in the limit, and the existence of all $n$-point functions on $\mathbb{P}^{1}$.

v. It is not hard to show that Cond. 4 is equivalent to a version of uniform convergence of the OPE-constants:

$$
\begin{gathered}
\forall \varphi \in \mathcal{H}^{\infty}, \forall \chi \in \mathcal{H}_{\alpha}^{\infty}, \alpha \in \mathcal{I}_{\infty}, \forall \mu, \bar{\mu} \in \mathbb{R}, \forall \varepsilon>0 \quad \exists I \in \mathbb{N}: \\
\forall i \geq I, \forall \psi \in V_{\mu, \bar{\mu}}(\varphi, \chi) \text { with } C^{\infty}\left(\psi^{*}, \varphi, \chi\right) \neq 0: \\
\left|C^{i}\left(\left(\psi^{i}\right)^{*}, \varphi^{i}, \chi^{i}\right)-C^{\infty}\left(\psi^{*}, \varphi, \chi\right)\right|<\varepsilon\left|C^{\infty}\left(\psi^{*}, \varphi, \chi\right)\right| .
\end{gathered}
$$

The above notion of full convergence turns out to be too restrictive for our purposes. In fact, we would like to allow for diverging conformal weights and other structure constants in decoupled sectors of the CFTs. This happens for example in the large radius limit of the free boson on the circle, where the winding modes get infinitely massive as $R \rightarrow \infty$, see (1.2.10). As motivated by Def. 1.3.2 in these cases we should restrict our considerations to the closed sectors with converging conformal weights: 
Definition 2.1.6

We call a sequence $\left(\widehat{\mathcal{C}}^{i}, \widehat{f}_{i}^{j}\right)$ of $C F T_{\mathrm{S}} \widehat{\mathcal{C}}^{i}=\left(\widehat{\mathcal{H}}^{i}, \widehat{*}^{i}, \Omega^{i}, T^{i}, \bar{T}^{i}, \widehat{C}^{i}\right)$ CONVERGENT, if the following holds:

For every $i \in \mathbb{N}$, the subspace $\mathcal{H}^{i} \subset \widehat{\mathcal{H}}^{i}$ consisting of those vectors whose conformal weights converge under the $\widehat{f}_{i}^{j}$ is closed under the OPE. Moreover,

$$
\forall i, j \in \mathbb{N}, i \leq j: \quad f_{i}^{j}:=\widehat{f}_{i}^{j} \mid \mathcal{H}^{i}, \quad C^{i}:=\widehat{C}_{\mid\left(\check{\mathcal{H}}^{*}\right)^{i} \otimes \mathcal{H}^{i} \otimes \mathcal{H}^{i}}, \quad *^{i}:=\widehat{*}_{\mid \mathcal{H}^{i}}^{i}
$$

defines a fully convergent system $\left(\mathcal{H}^{i}, C^{i}, f_{i}^{j}\right)$ with stable $W$-algebras $\mathcal{W}^{i} \supset$ $\mathcal{V} \operatorname{ir}_{c_{i}} \oplus \overline{\mathcal{V i r}}_{c_{i}}$. The corresponding direct limit

$$
\mathcal{C}^{\infty}:=\left(\mathcal{H}^{\infty}:=\left(\lim _{\longrightarrow} \mathcal{H}^{i}\right) / \mathcal{N}^{\infty}, *^{\infty}, \Omega, T, \bar{T}, C^{\infty}\right)
$$

is called Limit OF THE SEQUENCE $\left(\widehat{\mathcal{C}}^{i}, \widehat{f}_{i}^{j}\right)$ OF CFTs. The stable $\mathcal{W}$-algebras are called PRESERVED W-ALGEBRAS.

\section{Remark 2.1.7}

The discussion of convergence of sequences of CFTs generalizes to one-dimensional CFT-spaces $\mathcal{S}$ (see Def. 1.3.3). Instead of the homomorphisms $f_{i}^{j}$ we specify a connection (i.e. the parallel sections) on the sheaf $\mathcal{S}$. Sequences can then be defined on local trivializations of $\mathcal{S}$ by parallel transport. If such a sequence converges in the sense of Def. 2.1.6 then the limit structures discussed above give rise to a boundary point of the CFT-space.

For a general CFT-space $\mathcal{S}$ over $\mathcal{M}$ with non-compact $\mathcal{M}$, equipped with a flat connection (e.g. obtained from deformation theory), we can then construct boundary points as limits of convergent sequences which come from onedimensional CFT-subspaces as above. We will discuss such a boundary of the CFT-space of toroidal CFTs ${ }^{10}$ in Sect. 3.1

2.2. Geometric interpretations. As mentioned above, our notion of convergence admits the occurrence of degeneration phenomena. One of them is the VACUUM DEGENERACY, i.e. the degeneration of the subspace of states with vanishing conformal weights. While this subspace is one-dimensional in a well-defined CFT, it may become higher-dimensional, and even infinite-dimensional, in the limit of CFTs. In Def. 1.3.2 we have introduced PREFERRED GEOMETRIC INTERPRETATIONS of CFTs; in this section we will argue that limits of CFTs with an appropriate vacuum degeneracy can be expected to allow such geometric interpretations. Similar approaches have been proposed in [M-S1, §6] as well as [F-G] K-S, but with no general definitions of sequences and limits of CFTs at hand.

In the following, let $\left(\mathcal{C}^{i}\right)_{i \in \mathbb{N}}=\left(\mathcal{H}^{i}, *^{i}, \Omega^{i}, T^{i}, \bar{T}^{i}, C^{i}\right)_{i \in \mathbb{N}}$ denote a convergent sequence of CFTs. As in Def. [2.1.6] its limit is denoted $\mathcal{C}^{\infty}=\left(\mathcal{H}^{\infty}:=\right.$ $\left.\mathcal{K}^{\infty} / \mathcal{N}^{\infty}, *^{\infty}, \Omega, T, \bar{T}, C^{\infty}\right)$. By Cond. [5 we can set

$$
\begin{aligned}
& \widetilde{\mathbb{H}}^{\infty}:=f_{i}^{\infty}\left(\left(\mathcal{H}^{i}\right)^{\mathcal{V i r}}\right) \subset \mathcal{H}^{\infty}, \text { and } \mathbb{H}^{\infty}:=\operatorname{ker}\left(L_{0}\right) \cap \operatorname{ker}\left(\bar{L}_{0}\right)=\mathcal{H}_{0,0}^{\infty} \subset \mathcal{H}^{\infty} \text {. } \\
& \hline 10 \text { In fact, CFT-spaces of toroidal models, more generally of WZW- and coset-models (see } \\
& {[\text { F-R] or of orbifolds thereof, and discrete sequences of CFTs are the only well known examples }} \\
& \text { of CFT-spaces. Although the moduli space } \mathcal{M} \text { of } N=(4,4) \text { SCFTs on } K 3 \text { is known [A-M, } \\
& \text { the corresponding CFT-space } \mathcal{S} \text { over } \mathcal{M} \text { has not yet been constructed. }
\end{aligned}
$$


Note that $\mathbb{H}^{\infty} \subset \widetilde{\mathbb{H}}^{\infty}$, since descendants cannot have vanishing dimensions. To every $\varphi \in \mathbb{H}^{\infty}$ we associate an operator $A_{\varphi}$ on $\widetilde{\mathbb{H}}^{\infty}$ by truncation of the OPE, as before: By Conds. 4 and 7 for $\chi \in \widetilde{\mathbb{H}}^{\infty}$ we can copy (1.2.6) verbatim to define $\varphi$ 困 $\chi$. Then $A_{\varphi}(\chi):=\varphi$ 困 $\chi$. Let $\mathcal{A}^{\infty}$ denote the algebra generated by all these operators:

$$
\begin{gathered}
\forall \varphi \in \mathbb{H}^{\infty}: A_{\varphi}: \widetilde{\mathbb{H}}^{\infty} \longrightarrow \widetilde{\mathbb{H}}^{\infty}, A_{\varphi}(\chi):=\varphi \text { 米; } \mathcal{A}^{\infty}:=\left\langle A_{\varphi} \mid \varphi \in \mathbb{H}^{\infty}\right\rangle, \\
\text { where } \forall \psi \in \widetilde{\mathbb{H}}^{\infty}: \psi^{*}(\varphi \text { 国 } \chi)=C^{\infty}\left(\psi^{*}, \varphi, \chi\right) .
\end{gathered}
$$

We first collect some properties of $\mathcal{A}^{\infty}$ :

\section{Lemma 2.2.1}

In the limit of a sequence of CFTs, for every state $\varphi \in \mathbb{H}^{\infty}$ of vanishing conformal weights one has $L_{1} \varphi=\bar{L}_{1} \varphi=0$. Moreover, $A_{\varphi}$ preserves weights, i.e. with $\widetilde{\mathbb{H}}_{h, \bar{h}}^{\infty}:=\widetilde{\mathbb{H}}^{\infty} \cap \mathcal{H}_{h, \bar{h}}^{\infty}$, for all $h, \bar{h} \in \mathbb{R}$ we find $A_{\varphi}\left(\widetilde{\mathbb{H}}_{h, \bar{h}}^{\infty}\right) \subset \widetilde{\mathbb{H}}_{h, \bar{h}}^{\infty}$. In particular, $\mathcal{A}^{\infty}$ acts on $\mathbb{H}^{\infty}$.

Proof:

Fix $\varphi \in \mathbb{H}^{\infty}$. Note that $L_{1}^{i} \varphi^{i}$ converges weakly to $L_{1} \varphi$ by Cond. [6] Using this, we first show that in $\mathcal{K}^{\infty}=\lim \mathcal{H}^{i}, L_{1} \varphi$ is a null vector, i.e. $L_{1} \varphi \in \mathcal{N}^{\infty}$. Indeed,

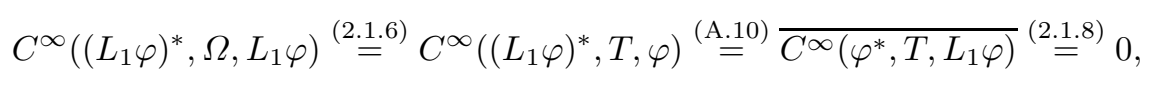

which by definition (2.1.2) proves $L_{1} \varphi \in \mathcal{N}^{\infty}$. Similarly, $\bar{L}_{1} \varphi \in \mathcal{N}^{\infty}$.

Using weak convergence, Cond. 2] and (A.14), since $\varphi$ has vanishing conformal weights, we find

$$
\forall \psi \in \mathcal{H}_{h_{\psi}, \bar{h}_{\psi}}^{\infty}, \chi \in \mathcal{H}_{h_{\chi}, \bar{h}_{\chi}}^{\infty}: \quad\left(h_{\psi}-h_{\chi}\right) C^{\infty}\left(\psi^{*}, \varphi, \chi\right)=C^{\infty}\left(\psi^{*}, L_{1} \varphi, \chi\right)=0 .
$$

Hence $C^{\infty}\left(\psi^{*}, \varphi, \chi\right)=\psi^{*}\left(A_{\varphi} \chi\right) \neq 0$ only if $h_{\chi}=h_{\psi}$, and similarly $\bar{h}_{\chi}=\bar{h}_{\psi}$. This proves the claim.

By the above, the only non-trivial mode of each $\varphi \in \mathbb{H}^{\infty}$ is $\varphi_{0,0}$. This motivates

\section{Definition 2.2.2}

In the limit $\mathcal{C}^{\infty}$ of a converging sequence of CFTs we set $\mathbb{H}^{\infty}:=\mathcal{H}_{0,0}^{\infty}$ and call $\mathcal{A}^{\infty}:=\left\langle A_{\varphi} \mid \varphi \in \mathbb{H}^{\infty}\right\rangle$ the ZERO MODE ALGEBRA.

To fix notations, we now choose an orthonormal basis $\left\{\psi_{j}\right\}_{j \in \mathbb{N}}$ of $\mathbb{H}^{\infty}$ such that $*\left(\psi_{j}\right)=\psi_{j}$ for all $j \in \mathbb{N}$ and $\psi_{j}^{i} \in \mathcal{H}_{h_{j}^{i}, h_{j}^{i}}^{i}$ where as always $\psi_{j}^{i}$ is a representative of $\psi_{j}$, i.e. $\psi_{j}=f_{i}^{\infty}\left(\psi_{j}^{i}\right)$ in $\mathcal{H}^{\infty}$. We set

$$
\forall a, b, c \in \mathbb{N}: \quad C_{a b}^{c}:=C^{\infty}\left(\psi_{c}^{*}, \psi_{a}, \psi_{b}\right) \stackrel{\text { A.10 }}{=} C_{a c}^{b} \stackrel{\mathrm{A.12}}{=} C_{c a}^{b} .
$$

Following [F-G], we expect the zero mode algebra of a limit of CFTs to give rise to a spectral triple which defines a commutative geometry. In fact, 


\section{Lemma 2.2.3}

The zero mode algebra $\mathcal{A}^{\infty}$ of the limit of a sequence of CFTs is commutative if and only if

$$
\forall \varphi, \chi \in \mathbb{H}^{\infty}: \quad A_{\varphi} \circ A_{\chi}=A_{\varphi \circledast \chi}
$$

Proof:

With respect to the orthonormal basis $\left\{\psi_{j}\right\}_{j \in \mathbb{N}}$ chosen before (2.2.1), we have

$$
\forall a, b \in \mathbb{N}: \quad \psi_{a} \text { 囝 } \psi_{b}=\sum_{j} C_{a b}^{j} \psi_{j} .
$$

One therefore checks:

$$
\begin{aligned}
& \forall a, b \in \mathbb{N}: \quad A_{\psi_{a}} \circ A_{\psi_{b}}=A_{\psi_{b}} \circ A_{\psi_{a}} \\
& \stackrel{2.2 .1}{\Longleftrightarrow} \sum_{j} C_{a d}^{j} C_{b c}^{j}=\sum_{j} C_{b d}^{j} C_{a c}^{j} \quad \forall c, d \in \mathbb{N} \\
& \stackrel{2.2 .1}{\Longleftrightarrow} \quad A_{\psi_{a}} \circ A_{\psi_{b}}=A_{\psi_{a} \text { 困 } \psi_{b}} \text {. }
\end{aligned}
$$

\section{Proposition 2.2.4}

The zero mode algebra $\mathcal{A}^{\infty}$ of the limit of a convergent sequence of CFTs is commutative.

Proof:

By the proof of Lemma 2.2.3 the claim is equivalent to (2.2.2). This equation follows from the relations imposed on the OPE-constants by crossing symmetry. Namely, for all $a, b, c, d \in \mathbb{N}$, both sides of

$$
\left\langle\psi_{d}^{i}\left|\psi_{c}^{i}(1,1) \psi_{a}^{i}(z, \bar{z})\right| \psi_{b}^{i}\right\rangle_{i}=\left\langle\psi_{b}^{i}\left|\psi_{c}^{i}(1,1) \psi_{a}^{i}\left(z^{-1}, \bar{z}^{-1}\right)\right| \psi_{d}^{i}\right\rangle_{i} z^{-2 h_{a}^{i} \bar{z}^{-2} \bar{h}_{a}^{i}}
$$

converge to real analytic functions on $\mathbb{C}-\{0,1\} \cong \mathbb{P}^{1}-\{0,1, \infty\}$ with power series expansions in $z, \bar{z} ; z^{-1}, \bar{z}^{-1}$, respectively. Since by Cond. 5 the sum over primaries in A.16 does not contain contributions from null vectors, we can use (A.16 - A.18) to analyze the structure of (2.2.3): Both sides converge to formal power series in $z, z^{-1}$, respectively, with non-negative integer exponents, only. Hence both sides must be constant, receiving only contributions from the leading terms in the conformal blocks. Then A.18 shows $\sum_{j} C_{a b}^{j} C_{c j}^{d}=\sum_{j} C_{a d}^{j} C_{c j}^{b}$ which by (2.2.1) is equivalent to (2.2.2).

\section{Remark 2.2.5}

i. Similarly to Rem. 2.1.5iv the proof of Prop. 2.2.4 simplifies considerably if null vectors are not present in $\mathcal{K}^{\infty}$. Then the proof of Lemma 2.2.3 shows $L_{1} \psi_{a}=0$ as an element of $\mathcal{K}^{\infty}$ for all $\psi_{a} \in \mathbb{H}^{\infty}$, such that for all $\psi_{a}, \psi_{b}, \psi_{c}, \psi_{d}$ $\in \mathbb{H}^{\infty}$ :

$$
0=\left\langle\psi_{d}\left|\psi_{c}(1,1) L_{1} \psi_{a}(z, \bar{z})\right| \psi_{b}\right\rangle=\frac{\partial}{\partial z}\left\langle\psi_{d}\left|\psi_{c}(1,1) \psi_{a}(z, \bar{z})\right| \psi_{b}\right\rangle
$$

In other words, all conformal blocks are constant, and crossing symmetry can be used directly to show Prop. 2.2.4 
ii. Our definitions easily generalize to the case where the central charges of the left and right handed Virasoro algebras do not coincide. Then the situation greatly simplifies if all CFTs under consideration are chiral: One immediately identifies $A_{\varphi}(\chi), \varphi, \chi \in \mathbb{H}^{\infty}$, with the normal ordered product of $\varphi$ and $\chi$. Since $\mathcal{H}^{\infty}=\mathcal{K}^{\infty} / \mathcal{N}^{\infty}$ with $\mathcal{N}^{\infty}$ containing the ideal generated by $L_{1} \mathbb{H}^{\infty}$, $\mathbb{H}^{\infty}$ belongs to Zhu's commutative associative algebra [Zh B-N] G-N], which is known to be isomorphic to the zero-mode algebra [B-N]. It would be desirable to generalize the notion of Zhu's algebra to non-chiral theories, and it would be interesting to know if such a notion can reproduce $\mathcal{A}^{\infty}$ in the limit of convergent sequences of CFTs.

By Prop. 2.2.4 limits of CFTs are naturally expected to possess preferred geometric interpretations:

\section{Definition 2.2.6}

Let $\mathcal{C}^{\infty}$ denote the limit of a convergent sequence of CFTs with limiting central charge $c$ and zero mode algebra $\mathcal{A}^{\infty}$. Let $N \in \mathbb{N}$ be maximal such that for all $\varphi \in \mathbb{H}^{\infty}$ with $\varphi=f_{i}^{\infty}\left(\varphi^{i}\right), \varphi^{i} \in \mathcal{H}_{h_{i}, \bar{h}_{i}}^{i}$ :

$$
\lambda_{\varphi}^{N}:=\lim _{i \rightarrow \infty} i^{N}\left(h_{i}+\bar{h}_{i}\right)<\infty, \quad H^{\infty} \varphi:=\lambda_{\varphi}^{N} \varphi
$$

Then the linear extension of $H^{\infty}$ is a self-adjoint operator $H^{\infty}: \mathbb{H}^{\infty} \longrightarrow \mathbb{H}^{\infty}$.

If there exist completions $\overline{\mathbb{H}}^{\infty}, \overline{\mathcal{A}}^{\infty}$ of $\mathbb{H}^{\infty}, \mathcal{A}^{\infty}$ such that $\left(\overline{\mathbb{H}}^{\infty}, H^{\infty}, \overline{\mathcal{A}}^{\infty}\right)$ is a spectral triple of dimension $c$, then the latter is called a GEOMETRIC INTERPRETATION OF $\mathcal{C}^{\infty}$.

The above definition may seem artificial, since we cannot prove a general result allowing to give geometric interpretations for arbitrary limits of CFTs. However, below we will see that there are interesting examples which do allow such geometric interpretations, in particular a non-standard one which we present in Sect. 4 Moreover, from the viewpoint of non-linear sigma model constructions and large volume limits of their underlying geometries, Def. 2.2.6 formalizes the expected encoding of geometry in CFTs, see M-S1, F-G $\mathrm{K}-\mathrm{S}$, which justifies our definition.

\section{Limits of conformal field theories: Simple examples}

This section consists in a collection of known examples, where we discuss limits of CFTs and their geometric interpretations in the language introduced in Sect. [2. Sects. 3.1 and 3.2 deal with toroidal CFTs and orbifolds thereof, respectively. We confirm that our techniques apply to these cases and that they yield the expected results. In particular, the discussion of toroidal CFTs fits our approach into the picture drawn in $\mathrm{F}-\mathrm{G} \mid \mathrm{K}-\mathrm{S}$.

3.1. Torus models. As a first set of examples, let us discuss bosonic toroidal CFTs. These are $\mathfrak{u}(1)^{d}$-WZW models, whose W-algebras contain $\mathfrak{u}(1)^{d} \oplus \overline{\mathfrak{u}(1)}^{d}$ - 
subalgebras generated by the modes of the respective $\mathfrak{u}(1)^{d} \cong \mathbb{R}^{d}$-valued currents. That is, (B.1) generalizes to

$$
\begin{gathered}
j^{k}(z)=\sum_{n \in \mathbb{Z}} a_{n}^{k} z^{n-1}, \quad \bar{\jmath}^{k}(\bar{z})=\sum_{n \in \mathbb{Z}} \bar{a}_{n}^{k} \bar{z}^{n-1}, \quad k=1, \ldots d, \\
{\left[a_{n}^{k}, a_{m}^{l}\right]=m \delta^{k l} \delta_{m+n, 0}, \quad\left[\bar{a}_{n}^{k}, \bar{a}_{m}^{l}\right]=m \delta^{k, l} \delta_{m+n, 0}, \quad\left[a_{n}^{k}, \bar{a}_{m}^{l}\right]=0 .}
\end{gathered}
$$

Holomorphic and antiholomorphic energy-momentum tensors can be obtained as $T=\frac{1}{2} \sum_{k}: j^{k} j^{k}$. $\bar{T}=\frac{1}{2} \sum_{k}: \bar{j}^{k} \bar{j}^{k}$. Their modes give rise to holomorphic and antiholomorphic Virasoro algebras with central charges $c=d$.

The pre-Hilbert space $\mathcal{H}_{\Gamma}$ of a toroidal $\mathrm{CFT}_{\Gamma}$ decomposes into irreducible lowest weight representations of $\mathfrak{u}(1)^{d} \oplus \overline{\mathfrak{u}(1)}^{d}$, which are completely characterized by their holomorphic and antiholomorphic $\mathfrak{u}(1)^{d} \oplus \overline{\mathfrak{u}(1)}^{d}$-charges $(Q ; \bar{Q}) \in \Gamma \subset$ $\mathbb{R}^{2 d}$ :

$$
\mathcal{H}_{\Gamma} \cong \bigoplus_{(Q ; \bar{Q}) \in \Gamma} \mathcal{V}_{Q}^{\mathfrak{u}(1)^{d}} \otimes \overline{\mathcal{V}}_{\bar{Q}}^{\mathfrak{u}(1)^{d}}
$$

The corresponding norm-1 lwvs $|Q ; \bar{Q}\rangle$ have conformal weights ${ }^{11}$

$$
h_{|Q ; \bar{Q}\rangle}=\frac{1}{2} Q^{2}, \quad \bar{h}_{|Q ; \bar{Q}\rangle}=\frac{1}{2} \bar{Q}^{2},
$$

and, by definition, the corresponding fields $V_{|Q ; \bar{Q}\rangle}(z, \bar{z})$ (see A.7) obey

$$
\begin{aligned}
& j^{k}(w) V_{|Q ; \bar{Q}\rangle}(z, \bar{z})=\frac{Q^{k} V_{|Q ; \bar{Q}\rangle}(z, \bar{z})}{(w-z)}+\text { reg. } \\
& \bar{\jmath}^{k}(\bar{w}) V_{|Q ; \bar{Q}\rangle}(z, \bar{z})=\frac{\bar{Q}^{k} V_{|Q ; \bar{Q}\rangle}(z, \bar{z})}{(\bar{w}-\bar{z})}+\text { reg. }
\end{aligned}
$$

The $n$-point functions of the $V_{|Q ; \bar{Q}\rangle}(z, \bar{z})$ reduce to products of the respective holomorphic and antiholomorphic conformal blocks

$$
\left\langle 0\left|V_{\left|Q_{1} ; \bar{Q}_{1}\right\rangle}\left(z_{1}, \bar{z}_{1}\right), \ldots, V_{\left|Q_{n} ; \bar{Q}_{n}\right\rangle}\left(z_{n}, \bar{z}_{n}\right)\right| 0\right\rangle_{\mathbb{P}^{1}}=\prod_{1 \leq i<j \leq n}\left(z_{i}-z_{j}\right)^{Q_{i} Q_{j}}\left(\bar{z}_{i}-\bar{z}_{j}\right)^{\bar{Q}_{i} \bar{Q}_{j}} .
$$

Recall that the right hand side gives a well-defined function for $z_{i}, \bar{z}_{i} \in \mathbb{C}$ away from the partial diagonals, iff all $Q_{i} Q_{j}-\bar{Q}_{i} \bar{Q}_{j} \in \mathbb{Z}$. Indeed, the charges $(Q ; \bar{Q})$ of lwvs in a toroidal CFT constitute an even integral selfdual Lorentzian lattice $\Gamma \subset \mathbb{R}^{d, d}$ of signature $(d, d)$, where the quadratic form is given by the double spin $2(h-\bar{h})$ of the respective state, and addition corresponds to fusion. We denote by $U^{d}$ the unique even integral selfdual Lorentzian lattice of signature $(d, d)$ and regard $\Gamma$ as image of an embedding ${ }^{12} \iota: U^{d} \stackrel{\sim}{\rightarrow} \Gamma \subset \mathbb{R}^{d, d}$ of $U^{d}$ into $\mathbb{R}^{d, d}$. Making use of the OPE (3.1.1) and contour integration, the $n$-point functions of descendants can also be extracted from (3.1.2). This procedure only involves derivation and multiplication by charges $Q^{k}, \bar{Q}^{k}$.

\footnotetext{
11 For $Q \in \mathbb{R}^{d}, Q^{2}$ denotes the standard quadratic form on $\mathbb{R}^{d}$.

12 The embedding is only specified up to automorphisms of $U^{d}$.
} 
To determine the OPE-constants of lwvs from (3.1.2) note that the overall sign on the right hand side of this equation depends on the ordering of the fields $V_{\left|Q_{i} ; \bar{Q}_{i}\right\rangle}\left(z_{i}, \bar{z}_{i}\right)$. This is due to the COCYCLE FACTORS which for $d=1$ have the simple form (B.6). To state a general formula, we first have to generalize (B.4). Given a toroidal CFT with charge lattice $\iota\left(U^{d}\right)=\Gamma \subset \mathbb{R}^{d, d}$, one can always find a lattice $\Lambda \subset \mathbb{R}^{d}$ of rank $d$ and a linear map $B: \mathbb{R}^{d} \longrightarrow\left(\mathbb{R}^{d}\right)^{*}$, such that with respect to appropriate coordinates on $\mathbb{R}^{d, d}$ the following holds: Denote by $\Lambda^{*} \subset\left(\mathbb{R}^{d}\right)^{*}$ the $\mathbb{Z}$-dual of $\Lambda$ and identify $\left(\mathbb{R}^{d}\right)^{*}$ with $\mathbb{R}^{d}$ by means of the standard scalar product on $\mathbb{R}^{d}$. Then $B$ is skew-symmetric and

$$
\Gamma=\left\{(Q ; \bar{Q})=\frac{1}{\sqrt{2}}(\mu-B \lambda+\lambda ; \mu-B \lambda-\lambda) \mid(\mu, \lambda) \in \Lambda^{*} \times \Lambda\right\} .
$$

Moreover,

$$
\text { for } \begin{aligned}
\left(Q^{(\prime)} ; \bar{Q}^{(\prime)}\right)= & \frac{1}{\sqrt{2}}\left(\mu^{(\prime)}-B \lambda^{(\prime)}+\lambda^{(\prime)} ; \mu^{(\prime)}-B \lambda^{(\prime)}-\lambda^{(\prime)}\right) \in \Gamma: \\
& C\left(\left|Q+Q^{\prime} ; \bar{Q}+\bar{Q}^{\prime}\right\rangle^{*},|Q ; \bar{Q}\rangle,\left|Q^{\prime} ; \bar{Q}^{\prime}\right\rangle\right)=(-1)^{\mu \lambda^{\prime}},
\end{aligned}
$$

with all other OPE-constants vanishing. Note that for $d=1$, Eq. (3.1.4 simplifies to (B.4) - B.5 due to the absence of the $B$-field.

By the above, a toroidal CFT $\mathcal{C}=\mathcal{C}_{\Gamma}$ is completely characterized by its charge lattice $\Gamma$, and thus the moduli space of these models is the NARAIN MODULI SPACE

$$
\mathcal{M}_{\text {Narain }}^{d} \cong \mathrm{O}(d, d, \mathbb{Z}) \backslash \mathrm{O}(d, d) / O(d) \times O(d)
$$

of even integral selfdual Lorentzian lattices in $\mathbb{R}^{d, d}$ C-E-N-T,Nar.

We will discuss sequences $\left(\mathcal{C}^{i}:=\mathcal{C}_{\Gamma_{i}}, f_{i}^{j}\right)$ of toroidal CFTs in $\mathcal{M}_{\text {Narain }}^{d}$ with stable W-algebra $\mathfrak{u}(1)^{d} \oplus \overline{\mathfrak{u}(1)}^{d}$ and fixed $\iota_{i}: U^{d} \rightarrow \Gamma_{i}$ such that

$$
\begin{aligned}
& \forall i, j, \in \mathbb{N}, \lambda \in U^{d}, P, \bar{P} \in \mathbb{C}\left[x_{1}^{1}, \ldots, x_{1}^{d}, x_{2}^{1}, \ldots, x_{2}^{d}, \ldots\right]: \\
& f_{i}^{j}: P\left(\left(a^{i}\right)_{m}^{k}\right) \bar{P}\left(\left(\bar{a}^{i}\right)_{n}^{l}\right)\left|\iota_{i}(\lambda)\right\rangle_{i} \longmapsto P\left(\left(a^{j}\right)_{m}^{k}\right) \bar{P}\left(\left(\bar{a}^{j}\right)_{n}^{l}\right)\left|\iota_{j}(\lambda)\right\rangle_{j} .
\end{aligned}
$$

This choice of sequence is natural from the point of view of deformation theory and completion of $\mathcal{M}_{\text {Narain }}^{d}$, see Rem. 2.1.7 Indeed, the OPE of lwvs is constant under the $f_{i}^{j}$ defined in (3.1.5), and OPE as well as all correlation functions of such sequences converge if and only if the lattices $\Gamma_{i}=\iota_{i}\left(U^{d}\right)$ converge in $\mathbb{R}^{d, d}$. In this case, 3.1.5 is fully convergent in the sense of Def. 2.1.3 Furthermore $\mathcal{N}^{\infty}=\{0\}$, which implies that the limiting $n$-point functions have the usual factorization properties (c.f. Rem. 2.1.5 iv). In fact, the lattices $\Gamma_{i}$ converge within $\mathcal{M}_{\text {Narain }}^{d}$, such that no degeneration phenomena occur. All $n$-point functions on surfaces with positive genus converge as well, and the limit of such a sequence is again a toroidal CFT with charge lattice $\Gamma_{\infty}=\lim _{i \rightarrow \infty} \Gamma_{i}$.

On the other hand, $\mathcal{M}_{\text {Narain }}^{d}$ is not complete, and a sequence of lattices $\Gamma_{i}$ may degenerate but still give a convergent sequence in the sense of Def.[2.1.6 Namely, given any primitive null-sublattice $N \subset U^{d}$ of rank $\delta \in \mathbb{N}$, one can construct convergent sequences such that the images $\iota_{i}\left(N^{\perp}\right)$ converge with $\iota_{i}(N)$ collapsing 
to $\{0\}$, while the images of lattice points in $U^{d}-N^{\perp}$ diverge. In fact, we can split $^{13}$

$$
U^{d}=N^{*} \oplus N^{\perp}=N^{*} \oplus N \oplus M,
$$

such that $N^{*}$ is null with $N^{*} \oplus N \cong U^{\delta}$. Then the lattice in the limit,

$$
\Gamma_{\infty}:=\lim _{i \rightarrow \infty} \iota_{i}\left(N^{\perp}\right)=\lim _{i \rightarrow \infty} \iota_{i}(M)
$$

is again an even integral selfdual Lorentzian lattice in $\mathbb{R}^{d, d}$, however with smaller rank: $\Gamma_{\infty} \cong U^{d-\delta}$.

Every sequence (3.1.5) showing this kind of degeneration is convergent in the sense of Def. 2.1.6 The limiting pre-Hilbert space is a $\mathfrak{u}(1)^{d} \oplus \overline{\mathfrak{u}}(1)^{d}$-module

$$
\mathcal{H}^{\infty} \simeq \mathcal{H}_{\Gamma_{\infty}} \otimes \bigoplus_{\lambda \in N} \mathcal{V}_{Q=0}^{\mathfrak{u}(1)^{\delta}} \otimes \overline{\mathcal{V}}_{\bar{Q}=0}^{\mathfrak{u}(1)^{\delta}}
$$

As before, we have $\mathcal{N}^{\infty}=\{0\}$ and therefore the usual factorization properties of the limiting $n$-point functions on the sphere. However, the degeneration of the lattice results in a diverging torus partition function, and the limit only has the structure of a CFT on surfaces of genus 0 with an infinitely degenerate vacuum sector.

In the spirit of Rem. 2.1.7 we regard these degenerate limits as boundary points of the CFT-space $\mathcal{S}$ over $\mathrm{O}(d, d) / \mathrm{O}(d) \times \mathrm{O}(d)$ of toroidal CFTs. The underlying Hausdorff space of such boundary points has a stratification

$$
\partial^{\operatorname{deg}}(\mathrm{O}(d, d) / \mathrm{O}(d) \times \mathrm{O}(d)) \cong \bigcup_{1 \leq \delta \leq d} \mathrm{O}(d-\delta, d-\delta) / \mathrm{O}(d-\delta) \times \mathrm{O}(d-\delta) .
$$

If one furthermore takes into account the possibly different speeds of degeneration, then one ends up with the compactification described in [K-S], which also includes higher boundary strata.

As explained in Sect. 1.2 we can extract a spectral pre-triple $(\widetilde{\mathbb{H}}, H, \widetilde{\mathcal{A}})$ with associative algebra $\widetilde{\mathcal{A}}$ from every CFT. For a toroidal CFT with charge lattice $\Gamma$, (3.1.4) and a direct generalization of the discussion in Ex. 1.2.2 lead us to the twisted group algebra

$$
\widetilde{\mathcal{A}}_{\Gamma} \cong \mathbb{C}_{\varepsilon}[\Gamma], \quad \varepsilon:\left((Q ; \bar{Q}),\left(Q^{\prime} ; \bar{Q}^{\prime}\right)\right) \longmapsto(-1)^{\mu \lambda^{\prime}},
$$

i.e.

$$
|Q ; \bar{Q}\rangle \circledast\left|Q^{\prime} ; \bar{Q}^{\prime}\right\rangle=(-1)^{\mu \lambda^{\prime}}\left|Q+Q^{\prime} ; \bar{Q}+\bar{Q}^{\prime}\right\rangle
$$

with notations as in (3.1.4). For any $N$ as above, the lwvs corresponding to elements in $N$ generate a commutative subalgebra $\widetilde{\mathcal{A}}_{N} \cong \mathbb{C}[N] \subset \widetilde{\mathcal{A}}_{\Gamma}$. Similarly to the one-dimensional case discussed in Sect. 1.3 restriction to $\widetilde{\mathcal{A}}_{N}$ gives a commutative geometry. In fact, $\widetilde{\mathcal{A}}_{N}$ is isomorphic to the algebra of smooth functions on a $\delta$-dimensional torus $T_{\iota(N)}=\mathbb{R}^{\delta} / 2 \pi \Lambda_{\delta}$, where $\Lambda_{\delta}$ is a lattice of rank $\delta$ such that $\iota(N) \subset \Gamma$ in (3.1.3) is described by restricting to lattice vectors with $(\mu, \lambda)=(\mu, 0), \mu \in \Lambda_{\delta}^{*} \subset \Lambda^{*}$.

\footnotetext{
13 Here, $\oplus$ denotes the direct sum, not the orthogonal direct sum.
} 
The zero mode algebra $\mathcal{A}^{\infty}$ associated to the degenerate vacuum sector of (3.1.7) (see Sect. 2.2) is isomorphic to $\widetilde{\mathcal{A}}_{N} \cong \mathbb{C}[N]$, whose closure is the algebra of smooth functions on a topological torus $T_{N}$. More precisely, each $\mu \in \Lambda_{\delta}^{*}$ corresponds to a function $T_{\iota(N)} \ni x \mapsto e^{i \mu(x)} \in \mathbb{C}$. It is an eigenfunction of the Laplacian on $T_{\iota(N)}$, equipped with the standard metric inherited from $\mathbb{R}^{\delta}$, with eigenvalue $\mu^{2}$. The latter goes to zero as $i \rightarrow \infty$.

Since $\mathcal{N}^{\infty}=\{0\}, \mathcal{A}^{\infty}$ also acts on the entire limiting pre-Hilbert space $\mathcal{H}^{\infty}$, which is a projective module of finite type over $\mathcal{A}^{\infty}$ and can therefore be regarded as the space of sections of a vector bundle over $T_{N} \cong \operatorname{Spec}\left(\mathcal{A}^{\infty}\right)$. Thus, in the limit we find a CFT-fibration over the moduli space $T_{N}$ of ground states of the limit theory as described in [K-S]. In fact, with a suitable choice of coordinates as in (3.1.3), the limiting structure of such degenerating sequences of toroidal CFTs can be regarded as LARGE VOLUME LIMIT, where $T_{\iota_{i}(N)}$ is the sequence of subtori obtaining infinite radii, while their duals collapse. It is a geometric interpretation in the sense of Def. 2.2.6 if $N$ is a maximal null sublattice, i.e. if $\delta=d$. Otherwise, it can be viewed as geometric interpretation of an appropriate subtheory with central charge $c^{\prime}=\delta$.

\section{Remark 3.1.1}

As described in Ex. 1.3.4 for the case of spectral triples of circles, we can also use partially ordered systems to obtain limits of toroidal CFTs different from the ones discussed above. For instance, in the case of circle models $\mathcal{C}_{R}, R \in \mathbb{R}^{+}$ (see Ex. 1.2.2 App. B), we can define a partially ordered system analogously to the construction given in Ex. 1.3.4 In the latter case, the limit spectral triple for functions on circles corresponds to $\mathbb{R}$ with a complicated topology. Similarly, the limit of the partially ordered system of circle theories decomposes into subsectors which only couple through the vacuum.

However, as explained in Rem. 1.3.5 one does not have to use the direct limit construction to define limits of spectral triples, and the same is true for limits of CFTs. Let us briefly discuss this in the case of the INFINITE RADIUS LIMIT OF CIRCLE MODELS.

To define a limit of the ordered set $\left(\mathcal{C}_{R}\right)_{R \in \mathbb{R}^{+}}$of CFTs without a given direct system, we have to find an appropriate Hermitean limit vector space $\mathcal{H}^{\infty}$ and epimorphisms $f_{R}: \mathcal{H}^{\infty} \rightarrow \mathcal{H}_{R}, R \in \mathbb{R}^{+}$, by hand. In fact, using notations as in (1.2.9),

$$
\mathcal{H}^{\infty}:=C_{c}^{\infty}(\mathbb{R}) \otimes \mathbb{C}\left[x_{1}, x_{2}, \ldots\right]^{\otimes 2}
$$

together with

$$
f_{R}(\chi \otimes P \otimes \bar{P}):=\frac{1}{\sqrt{R}} \sum_{m, n} \chi\left(\sqrt{2} Q_{m, n}\right) P\left(a_{r}\right) \bar{P}\left(\bar{a}_{s}\right)\left|Q_{m, n} ; \bar{Q}_{m, n}\right\rangle
$$

satisfy conditions similar to those stated in Rem. 1.3.5 Since the respective limits exist, we can define limit correlation functions by

$$
\left\langle 0\left|\varphi_{1}\left(z_{1}, \bar{z}_{1}\right) \ldots \varphi_{n}\left(z_{n}, \bar{z}_{n}\right)\right| 0\right\rangle:=\lim _{R \rightarrow \infty}\left\langle 0\left|f_{R}\left(\varphi_{1}\right)\left(z_{1}, \bar{z}_{1}\right) \ldots f_{R}\left(\varphi_{n}\right)\left(z_{n}, \bar{z}_{n}\right)\right| 0\right\rangle_{R},
$$

and similarly obtain limit OPE-constants.

The notion of convergence of sequences of CFTs introduced in Defs. 2.1.3 2.1.6 can be generalized to such a limit construction of ordered sets of CFTs. 
Indeed, the system $\left(\mathcal{C}_{R}, f_{R}\right)_{R \in \mathbb{R}^{+}}$defined by (3.1.9) - 3.1.10) is convergent in this generalized sense. Its limit is a full CFT, namely the UNCOMPACTIFIED FREE BOSONIC THEORY with pre-Hilbert space ${ }^{14}$

$$
\mathcal{H}=\bigoplus_{Q \in \mathbb{R}} \mathcal{V}_{Q}^{\mathfrak{u}(1)} \otimes \overline{\mathcal{V}}_{Q}^{\mathfrak{u}(1)}
$$

and in particular does not show any degeneration phenomena. We emphasize that $\mathcal{H}^{\infty}$ and the $f_{R}$ had to be constructed by hand and are not compatible with CFT-deformation theory, which is our reason for preferring the direct limit construction of Sect. 2

3.2. Torus orbifolds. If a given CFT allows an appropriate action of a finite symmetry group ${ }^{15}$, then one can construct a new model from these data by ORBIFOLDING, see e.g. D-V-V-V]. Since from our point of view the main ideas are apparent already in the simplest examples of torus orbifold models, namely the $\mathbb{S}^{1} / \mathbb{Z}_{2}$-orbifold theories $\mathcal{C}_{R}^{\mathbb{Z}_{2}}, R \in \mathbb{R}^{+}$, that is the $\mathbb{Z}_{2}$-orbifolds of the circle models $\mathcal{C}_{R}$ described in Ex. 1.2.2 and App. B] we will restrict our discussion to this family.

On the pre-Hilbert space $\mathcal{H}_{R}$ of the circle theory $\mathcal{C}_{R}$, the non-trivial element of $\mathbb{Z}_{2}$ acts by

$$
P\left(a_{n}\right) \bar{P}\left(\bar{a}_{n}\right)\left|Q_{R} ; \bar{Q}_{R}\right\rangle \longmapsto P\left(-a_{n}\right) \bar{P}\left(-\bar{a}_{n}\right)\left|-Q_{R} ;-\bar{Q}_{R}\right\rangle
$$

for $P, \bar{P} \in \mathbb{C}\left[x_{1}, x_{2}, \ldots\right]$. The pre-Hilbert space of the resulting orbifold model $\mathcal{C}_{R}^{\mathbb{Z}_{2}}$ consists of the $\mathbb{Z}_{2}$-invariant part of $\mathcal{H}_{R}$ and additional twisted sectors. Each sector decomposes into lowest-weight representations of the generic orbifold Walgebra $\mathcal{W}=W(2,4) \oplus \overline{W(2,4)} \subset \mathfrak{u}(1) \oplus \overline{\mathfrak{u}(1)}$ as detailed in Nah]: In the untwisted sector, there are norm-1 lwvs $\frac{1}{\sqrt{2}}\left|Q_{R} ; \bar{Q}_{R}\right\rangle^{\mathbb{Z}_{2}}=\frac{1}{\sqrt{2}}\left|-Q_{R} ;-\bar{Q}_{R}\right\rangle^{\mathbb{Z}_{2}}$ of conformal weights $h=\frac{1}{2} Q_{R}^{2}, \bar{h}=\frac{1}{2} \bar{Q}_{R}^{2}$ for each $\mathbb{Z}_{2}$-equivalence class of charges (B.4) appearing in the original circle theory. An additional norm-1 lwv $\left|\Theta_{R}\right\rangle$ of conformal weights $h=\bar{h}=1$ occurs in the basic $\mathfrak{u}(1) \oplus \overline{\mathfrak{u}(1)}$ representation with $Q_{R}=\bar{Q}_{R}=0$. Furthermore, in the twisted sector, there are four lwvs $\left|\sigma_{R}^{l}\right\rangle,\left|\tau_{R}^{l}\right\rangle$, $l \in\{0,1\}$, with $h=\bar{h}=\frac{1}{16}, h=\bar{h}=\frac{9}{16}$, respectively.

The OPE in the circle theories is invariant under the $\mathbb{Z}_{2}$-action, and the OPE in the orbifold models respects the $\mathbb{Z}_{2}$-grading on the pre-Hilbert spaces. Hence the correlation functions and OPE of states in the invariant sectors of the orbifold models coincide with the respective data in the circle theories. Correlation functions containing states in the twisted sectors have been discussed in D-F-M-S D-V-V], and the OPE between lwvs can be extracted from them.

Given a sequence $\left(R_{i}\right)_{i \in \mathbb{N}}$ in $\mathbb{R}^{+}$, we consider the sequence $\left(\mathcal{C}_{R_{i}}^{\mathbb{Z}_{2}}, f_{i}^{j}\right)$ of $\mathbb{S}^{1} / \mathbb{Z}_{2^{-}}$ orbifold models such that on lwvs $f_{i}^{j}\left(\left|Q_{R_{i}} ; \bar{Q}_{R_{i}}\right\rangle^{\mathbb{Z}_{2}}\right)=\left|Q_{R_{j}} ; \bar{Q}_{R_{j}}\right\rangle^{\mathbb{Z}_{2}}, f_{i}^{j}\left(\left|\Theta_{R_{i}}\right\rangle\right)=$

\footnotetext{
14 This pre-Hilbert space is a closure of $\mathcal{H}^{\infty}$ defined in 3.1.9.

15 That is, the group acts as group of automorphisms on the pre-Hilbert space of our theory leaving the n-point functions invariant, and the level matching conditions $\mathrm{D}-\mathrm{H}-\mathrm{V}-\mathrm{W}$ are obeyed.
} 
$\left|\Theta_{R_{j}}\right\rangle, f_{i}^{j}\left(\left|\sigma_{R_{i}}^{l}\right\rangle\right)=\left|\sigma_{R_{j}}^{l}\right\rangle, f_{i}^{j}\left(\left|\tau_{R_{i}}^{l}\right\rangle\right)=\left|\tau_{R_{j}}^{l}\right\rangle$. This definition naturally extends to the descendants as in (3.1.5).

Then, as in the case of toroidal models, all correlation functions and the OPE converge with respect to the $f_{i}^{j}$ if and only if $\left(R_{i}\right)_{i \in \mathbb{N}}$ converges in $\mathbb{R}^{+}$, $\lim _{i \rightarrow \infty} R_{i}=R_{\infty}>0$. In this case, $\left(\mathcal{C}_{R_{i}}^{\mathbb{Z}_{2}}, f_{i}^{j}\right)$ is a fully convergent sequence of CFTs in the sense of Def. 2.1.3 and $\mathcal{N}^{\infty}=\{0\}$, implying the existence of correlation functions on $\mathbb{P}^{1}$ (see Rem. 2.1.5 iv). No degeneration occurs, which means that correlation functions on surfaces of positive genus converge, too. Thus, in the limit we obtain a full CFT, namely the $\mathbb{S}^{1} / \mathbb{Z}_{2}$-model at radius $R_{\infty}$.

If $R_{i} \rightarrow 0$ or $R_{i} \rightarrow \infty$, our sequence of CFTs is convergent in the sense of Def. [2.1.6] Indeed, all correlation functions between states with convergent weights converge, $\mathcal{N}^{\infty}=\{0\}$, and in the limit we obtain a well-defined CFT on the sphere with degenerate vacuum sector. In the language of Sect. 3.1 for $R_{i} \rightarrow \infty$ we can use (3.1.6) with $M=\{0\}$ and $N=\left\{\frac{1}{\sqrt{2}}(m ; m) \mid m \in \mathbb{Z}\right\}$, $N^{*}=\left\{\frac{1}{\sqrt{2}}(n ;-n) \mid n \in \mathbb{Z}\right\}$, and $N \leftrightarrow N^{*}$ if $R_{i} \rightarrow 0$.

By Prop. 1.2.1 we can associate a spectral pre-triple $(\widetilde{\mathbb{H}}, H, \widetilde{\mathcal{A}})$ to each orbifold model $\mathcal{C}_{R}^{\mathbb{Z}_{2}}$. As mentioned after (1.2.6), here we find $\mathcal{H}^{\mathcal{W}} \supsetneqq \mathcal{H}^{\mathcal{W}}$. By D-F-M-S $\mathrm{D}-\mathrm{V}-\mathrm{V}]$ the OPE-constants including twisted ground states are given by

$$
\begin{aligned}
C^{R}\left(\left|\sigma_{R}^{k}\right\rangle^{*},\left|Q_{m, n} ; \bar{Q}_{m, n}\right\rangle^{\mathbb{Z}_{2}},\left|\sigma_{R}^{l}\right\rangle\right) \stackrel{\text { A.10], }}{=} C^{R}\left(\left(\left|Q_{m, n} ; \bar{Q}_{m, n}\right\rangle^{\mathbb{Z}_{2}}\right)^{*},\left|\sigma_{R}^{k}\right\rangle,\left|\sigma_{R}^{l}\right\rangle\right) \\
=\frac{2(-1)^{m l} \delta_{n+l, k}}{2^{Q_{m, n}^{2}+\bar{Q}_{m, n}^{2}}}
\end{aligned}
$$

with notations as in (1.2.9). Hence the $I_{\mathcal{W}}\left(\sigma_{R}^{k}, \sigma_{R}^{l}\right)$ used in (1.2.6) are infinite. On the other hand, $\widetilde{\mathcal{A}}$ contains a subalgebra given by the $\mathbb{Z}_{2}$-invariant part $\widetilde{\mathcal{A}}^{\prime}:=\mathbb{C}_{\varepsilon}[\Gamma]^{\mathbb{Z}_{2}}$ of the respective algebra of the underlying circle theory, c.f. (3.1.8). $\left|\Theta_{R}\right\rangle$ acts on $\widetilde{\mathcal{A}}^{\prime}$ as a second order differential operator, and $\widetilde{\mathcal{A}}$ is an $\widetilde{\mathcal{A}}^{\prime}$ module. Thus, $\widetilde{\mathcal{A}}$ can be regarded as the space of sections of a sheaf over the noncommutative space associated to the restricted spectral pre-triple $\left(\widetilde{\mathbb{H}}^{\prime}, H^{\prime}, \widetilde{\mathcal{A}}^{\prime}\right)$.

If $R_{i} \rightarrow \infty$, the zero mode algebra $\mathcal{A}^{\infty} \cong \mathbb{C}[\mathbb{Z}]^{\mathbb{Z}_{2}}$ is generated by the lwvs represented by $\left|\frac{m}{\sqrt{2} R_{i}} ; \frac{m}{\sqrt{2} R_{i}}\right\rangle^{\mathbb{Z}_{2}}, m \in \mathbb{Z}$. It is the algebra of $\mathbb{Z}_{2}$-symmetric functions on the circle, i.e. the functions on $\mathbb{S}^{1} / \mathbb{Z}_{2}$. In fact, the $|m\rangle_{\infty}^{\mathbb{Z}_{2}}:=f_{i}^{\infty}\left(\left|\frac{m}{\sqrt{2} R_{i}} ; \frac{m}{\sqrt{2} R_{i}}\right\rangle^{\mathbb{Z}_{2}}\right)$ are characterized by the recursion relation

$$
|m+1\rangle_{\infty}^{\mathbb{Z}_{2}}=|m\rangle_{\infty}^{\mathbb{Z}_{2}} ⿴|1\rangle_{\infty}^{\mathbb{Z}_{2}}-|m-1\rangle_{\infty}^{\mathbb{Z}_{2}},
$$

which agrees with the recursion relation for the (rescaled) CHEByshev POLYNOMIALS OF THE FIRST KIND, see e.g. [He]

$$
T_{m}(\cos x):=2 \cos (m x), \quad \text { for } m \in \mathbb{N}, x \in[0, \pi] .
$$

Hence $|m\rangle_{\infty}^{\mathbb{Z}_{2}}$ should be identified with the function $x \mapsto T_{m}(\cos x)$. This is not surprising, since the lwvs $|m\rangle_{\infty}^{\mathbb{Z}_{2}}$ are $\mathbb{Z}_{2}$-symmetric combinations of lwvs in the underlying circle theories, which in turn correspond to exponential functions.

Indeed, $\left\{T_{0} / \sqrt{2}, T_{1}, T_{2}, \ldots\right\}$ is an orthonormal basis of $L^{2}\left([0, \pi]\right.$, dvol $\left._{g}\right)$ with $\operatorname{dvol}_{g}=d x / 2 \pi$, i.e. with the flat standard metric $g$ on $[0, \pi] \cong \mathbb{S}^{1} / \mathbb{Z}_{2}$. Hence the 
methods of Sect. 1.1 yield $\overline{\mathbb{H}^{\infty}}=L^{2}\left(\mathbb{S}^{1} / \mathbb{Z}_{2}, d x / 2 \pi\right), \overline{\mathcal{A}^{\infty}}=C^{\infty}\left(\mathbb{S}^{1} / \mathbb{Z}_{2}\right)$, which according to Def. [2.2.6] for the limit gives the expected geometric interpretation on $\mathbb{S}^{1} / \mathbb{Z}_{2}$ with the flat metric $g$ induced from the standard metric on $\mathbb{S}^{1}$ and a trivial dilaton $\Phi$. Note also that the $m^{\text {th }}$ Chebyshev polynomial $T_{m}$ is an eigenfunction of the Laplacian $\frac{1}{2} \Delta_{g}=-\frac{1}{2} \frac{d^{2}}{d x^{2}}$ with eigenvalue $\frac{1}{2} m^{2}$, as expected from

$$
\begin{aligned}
H^{\infty}|m\rangle_{\infty}^{\mathbb{Z}_{2}} & =\lambda_{m}^{2}|m\rangle_{\infty}^{\mathbb{Z}_{2}} \\
& \text { with } \quad \lambda_{m}^{2}=\lim _{i \rightarrow \infty} R_{i}^{2}\left(h_{\left|Q_{m, 0} ; \bar{Q}_{m, 0}\right\rangle}^{i}+\bar{h}_{\left|Q_{m, 0} ; \bar{Q}_{m, 0}\right\rangle}^{i}\right)=\frac{1}{2} m^{2} .
\end{aligned}
$$

As for the toroidal CFTs discussed in Sect. 3.1] $\mathcal{A}^{\infty}$ acts on the entire pre-Hilbert space $\mathcal{H}^{\infty}$ which can be regarded as the space of sections of a sheaf over $\mathbb{S}^{1} / \mathbb{Z}_{2}$. Let us restrict the discussion to the states $\left|\sigma^{l}\right\rangle_{\infty}:=f_{i}^{\infty}\left(\left|\sigma_{R_{i}}^{l}\right\rangle\right)$. The action of $\mathcal{A}^{\infty}$ on them can be extracted from the OPE-coefficients (3.2.1):

$$
\begin{aligned}
\left|Q_{m, n} ; \bar{Q}_{m, n}\right\rangle^{\mathbb{Z}_{2}}\left|\sigma_{R}^{l}\right\rangle & =\frac{2(-1)^{m l}}{2^{Q_{m, n}^{2}+\bar{Q}_{m, n}^{2}}}\left|\sigma_{R}^{l+n}\right\rangle \\
& \Longrightarrow \quad|m\rangle_{\infty}^{\mathbb{Z}_{2}}\left|\sigma^{l}\right\rangle_{\infty}=2(-1)^{m l}\left|\sigma^{l}\right\rangle_{\infty} .
\end{aligned}
$$

It follows that the sections corresponding to $\left|\sigma_{R}^{l}\right\rangle$ are peaked around the respective $\mathbb{Z}_{2}$-fixed points, i.e. the endpoints of the interval $[0, \pi]$. In the limit their support in fact shrinks to these points. The same holds true for all other states in the twisted sectors. They are sections of skyscraper sheaves over the fixed points of the orbifold action. As expected, in the limit the OPE of two states in the twisted sectors vanishes, unless the corresponding sections have common support. This gives a nice geometric interpretation of the twisted sectors.

\section{The $m \rightarrow \infty, c \rightarrow 1$ limit of the unitary Virasoro minimal models $\mathcal{M}(m, m+1)$}

The present section contains the main results of this work: In Sect. 4.1 we show that the techniques introduced in Sect. 2 for the study of limits and degeneration phenomena also apply to the family of diagonal unitary Virasoro minimal models $\mathcal{M}(m, m+1), m \in \mathbb{N}-\{0,1\}$, which gives a fully convergent sequence of CFTs. In Sect. 4.2 we determine and study a geometric interpretation of its limit $\mathcal{M}_{\infty}$ as $m \rightarrow \infty$, and we discuss the inherent D-brane geometry.

4.1. The unitary Virasoro minimal models $\mathcal{M}(m, m+1)_{m \rightarrow \infty}$. Both outset and favorite example for our investigation are the unitary Virasoro minimal models $\mathcal{M}_{m}:=\mathcal{M}(m, m+1), m \in \mathbb{N}-\{0,1\}$ B-P-Z, which correspond to the $(A, A)$ (left-right symmetric) modular invariant partition functions in the CIZ classification C-I-Z2 C-I-Z1]. In this section, we explain how a fully convergent sequence $\left(\mathcal{C}^{m}, f_{m}^{j}\right)$ with $\mathcal{C}^{m}=\mathcal{M}_{m}$ for $m \in \mathbb{N}-\{0,1\}$ can be defined according to Def. [2.1.3 To our knowledge, such a construction was first alluded to in D-F1, §6 and App. B] as well as in [M-S1, §6]. Our approach also allows us to determine a geometric interpretation of the limit of this sequence as $m \rightarrow \infty$, according to Def. 2.2.6 
Let us start by recalling some of the main properties of the CFT $\mathcal{M}_{m}$. Since this model is diagonal, we can restrict our discussion to the action of the holomorphic Virasoro algebra. The pre-Hilbert space of $\mathcal{M}_{m}$ decomposes into a finite sum of irreducible representations of the Virasoro algebra $\mathcal{V i r}_{c_{m}}$ with central charge

$$
c_{m}=1-\frac{6}{m(m+1)} .
$$

These irreducible representations are labeled by $\mathcal{N}_{m}=\{(r, s) \mid r, s \in \mathbb{N}, 1 \leq r<$ $m, 1 \leq s<m+1\} / \sim$ with $(r, s) \sim(m-r, m+1-s)$, i.e. $r+s \sim 2 m+1-r-s$, such that by choosing appropriate representatives we can write

$$
\mathcal{N}_{m}=\{(r, s) \mid 1 \leq r<m, 1 \leq s<m+1, r+s \leq 2 m+1-r-s\} .
$$

Each irreducible Virasoro module $\mathcal{V}_{(r, s)}^{m},(r, s) \in \mathcal{N}_{m}$, has an lwv $|r, s\rangle_{m}$ of conformal dimension

$$
h_{(r, s)}^{m}=\frac{(r(m+1)-s m)^{2}-1}{4 m(m+1)} \quad \underset{\sim \rightarrow \infty}{\sim} \quad \frac{(r-s)^{2}}{4}+\frac{r^{2}-s^{2}}{4 m}+\frac{s^{2}-1}{4 m^{2}}+\cdots .
$$

We choose the $|r, s\rangle_{m}$ to be orthonormal.

The $n$-point functions for $\mathcal{M}_{m}$ are discussed in [D-F1], in particular all OPEcoefficients $C^{m}$ are determined in D-F1 D-F2], see App. [C] The calculations make use of the Feigin-Fuks integral representation [F-F] of $n$-point functions, assuming that $\mathcal{M}_{m}$ has a Coulomb-gas representation. That the latter is indeed true is shown in [Fe1].

To construct a sequence of CFTs according to Def. 2.1.1 we note that there are well-defined embeddings ${ }^{16}$

$$
\mathcal{N}_{m} \hookrightarrow \mathcal{N}_{m+1}, \quad(r, s) \longmapsto(r, s) .
$$

We will extend these embeddings to vector space homomorphisms $f_{m}^{m+1}$ between the corresponding irreducible Virasoro modules. To meet Cond. [5] of Sect. 2.1] we must map lwvs to lwvs:

$$
\begin{aligned}
\mathcal{V}_{(r, s)}^{m} & \hookrightarrow \mathcal{V}_{(r, s)}^{m+1} \\
P\left(L_{n}^{m}\right)|r, s\rangle_{m} & \longmapsto \widetilde{P}\left(L_{n}^{m+1}\right)|r, s\rangle_{m+1},
\end{aligned}
$$

similarly to (3.1.5). Here, $P, \widetilde{P}$ are elements of the same degree in the weighted polynomial ring $\mathbb{C}\left[x_{1}, x_{2}, \ldots\right]$ with $\operatorname{deg} x_{n}=n$, and we substitute $x_{n}=L_{n}^{m}$ or $x_{n}=L_{n}^{m+1}$ in lexicographical order (see Def. 4.1.1).

To construct consistent maps of type (4.1.4), recall from B-P-Z that the characteristic feature of the representation $\mathcal{V}_{(r, s)}^{m}$ is the fact that the Verma module built by the action of the Virasoro algebra $\mathcal{V i r}_{c_{m}}$ on $|r, s\rangle_{m}$ with character $q^{1-c_{m} / 24} \chi_{h_{(r, s)}}^{\text {gen }}(q)$ and $\chi_{h}^{\text {gen }}$ as in (B.7) contains a proper non-trivial submodule of SINGULAR VECTORS, that is of lwvs of $\mathcal{V i r}_{c_{m}}$ at positive level. The occurrence of these singular vectors, which have been quotiented out to obtain $\mathcal{V}_{(r, s)}^{m}$,

16 Our choice of embeddings is quite natural and has been used already in [Za2] in the context of slightly relevant perturbations of $\mathcal{M}_{m}$. However, there are other choices, leading to different limits of CFTs. 
makes our construction slightly delicate. However, the very properties of direct limits allow us to solve this problem. For later convenience, we give the following technical

\section{Definition 4.1.1}

Let $m \in \mathbb{N}-\{0,1\}$ and $(r, s) \in \mathcal{N}_{m}$. For each $N \in \mathbb{N}$ choose a set $\mathcal{P}_{(r, s)}^{m}(N)$ of monomials with weighted degree $N$, such that

$$
\left\{P\left(L_{n}^{m}\right)|r, s\rangle_{m} \mid P \in \mathcal{P}_{(r, s)}^{m}(N), N \in \mathbb{N}\right\}
$$

is a basis of $\mathcal{V}_{(r, s)}^{m}$, where for $P \in \mathcal{P}_{(r, s)}^{m}(N), P\left(x_{n}\right)=\prod_{n} x_{n}^{a_{n}}$ with $a_{i} \in \mathbb{N}$ and $\sum_{n} a_{n} \cdot n=N$,

If $B_{(r, s)}^{m} \in \mathbb{N}$ obeys

$$
P\left(L_{n}^{m}\right):=\left(L_{1}^{m}\right)^{a_{1}} \circ\left(L_{2}^{m}\right)^{a_{2}} \circ \cdots .
$$

$\forall N, N^{\prime} \in \mathbb{N}: \quad N+N^{\prime}<B_{(r, s)}^{m} \Longrightarrow \mathcal{P}_{(r, s)}^{m}(N) \cdot \mathcal{P}_{(r, s)}^{m}\left(N^{\prime}\right) \subset \mathcal{P}_{(r, s)}^{m}\left(N+N^{\prime}\right)$,

then $B_{(r, s)}^{m} \in \mathbb{N}$ is called an ENERGY BOUnd of $\mathcal{P}_{(r, s)}^{m}=\left(\mathcal{P}_{(r, s)}^{m}(N)\right)_{N \in \mathbb{N}}$. A system $\mathcal{P}_{(r, s)}^{m}$ with maximal energy bound among all systems giving bases 4.1.5) of $\mathcal{V}_{(r, s)}^{m}$ is called a BASIC MONOMIAL SYSTEM OF WEIGHT $h_{(r, s)}^{m}$. A sequence $\left(\mathcal{P}_{(r, s)}^{m}\right)_{m \geq M}$ of monomial systems is called SPECIAL if for all $m \geq M$ and for all $N<B_{(r, s)}^{m}$ : $\mathcal{P}_{(r, s)}^{m}(N)=\mathcal{P}_{(r, s)}^{m+1}(N)$, where $B_{(r, s)}^{m}$ are the respective energy bounds, and almost all $\mathcal{P}_{(r, s)}^{m}$ are basic.

Note that the relations which arise from the existence of singular vectors in the Verma module over $|r, s\rangle_{m}$, up to a global pre-factor $(m(m+1))^{-K}$ with $K \in \mathbb{N}$, are linear with respect to all monomials $P\left(L_{n}^{m}\right)$ of a given weighted degree $N$, with coefficients $a_{P} \in \mathbb{R}[m]$ of degree at most $2 N$. Moreover, as follows from the explicit character formula (C.1), the singular vectors which under the action of $\mathcal{V i r}_{c_{m}}$ generate the submodules of singular vectors have weights $h_{(r+m,-s+m+1)}^{m}$ and $h_{(r,-s+2(m+1))}^{m}$, i.e. levels $r s$ and $(m-r)(m+1-s)$, respectively. We conclude that for fixed $r, s \in \mathbb{N}-\{0\}$, the energy bound of basic monomial systems $\mathcal{P}_{(r, s)}^{m}$ of weights $h_{(r, s)}^{m}$ is monotonic increasing in $m$. Moreover,

\section{Lemma 4.1.2}

For every pair $r, s \in \mathbb{N}-\{0\}$ with $(r, s) \in \mathcal{N}_{M}$ ( $M$ minimal), we can choose a special sequence $\left(\mathcal{P}_{(r, s)}^{m}\right)_{m \geq M}$ of monomial systems according to Def. 4.1.1 and the respective energy bounds approach infinity as $m \rightarrow \infty$.

In the following, $\left(\mathcal{P}_{(r, s)}^{m}\right)_{m \geq M}$ will always denote a fixed special sequence of monomial systems of weights $h_{(r, s)}^{m}$ as in Lemma 4.1.2 Note that we can depict these monomial systems in terms of a convex polyhedron, as is customary in toric geometry. We then define

$$
\forall N \in \mathbb{N}, \forall P \in \mathcal{P}_{(r, s)}^{m}(N): \quad f_{m}^{m+1}\left[P\left(L_{n}^{m}\right)|r, s\rangle_{m}\right]:=P\left(L_{n}^{m+1}\right)|r, s\rangle_{m+1} .
$$

Finally, we linearly extend the $f_{m}^{m+1}$ to vector space homomorphisms

$$
f_{m}^{m}:=\operatorname{id}_{\mathcal{V}_{(r, s)}^{m}} ; f_{m}^{m+1}: \mathcal{V}_{(r, s)}^{m} \hookrightarrow \mathcal{V}_{(r, s)}^{m+1} ; f_{m}^{j}:=f_{j-1}^{j} \circ \cdots \circ f_{m}^{m+1}: \mathcal{V}_{(r, s)}^{m} \hookrightarrow \mathcal{V}_{(r, s)}^{j} .
$$


Then by construction,

\section{Lemma 4.1.3}

The sequence $\left(\mathcal{M}_{m}, f_{m}^{j}\right)$ is a sequence of CFTs with stable Virasoro algebra according to Defs. 2.1.1 and 2.1.2

In the following, we show that the sequence $\left(\mathcal{M}_{m}, f_{m}^{j}\right)$ is fully convergent according to Def. 2.1.3 Although above we have made a lot of choices, we will argue that our limit is independent of all choices, including the use of monomials and lexicographical order for their interpretation.

First note that by (4.1.1) and (4.1.3),

$$
c_{m} \stackrel{m \rightarrow \infty}{\longrightarrow} c=1, \quad h_{(r, s)}^{m} \stackrel{m \rightarrow \infty}{\longrightarrow} h_{(r, s)}=\frac{(r-s)^{2}}{4},
$$

i.e. all structure constants of the stable Virasoro algebras $\mathcal{V}_{i_{c_{m}}}$ converge. Moreover, setting

$$
C_{\left(n^{\prime}, n\right),\left(s^{\prime}, s\right)}^{\left(p^{\prime},{ }^{\prime}\right.}:=C^{m}\left(\left(\left|p^{\prime}, p\right\rangle_{m}\right)^{*},\left|n^{\prime}, n\right\rangle_{m},\left|s^{\prime}, s\right\rangle_{m}\right)
$$

with respect to orthonormal $|r, s\rangle_{m}$ as in D-F3, our calculations (C.3)-(C.8) imply

$$
C_{\left(n^{\prime}, n\right)\left(s^{\prime}, s\right)}^{\left(p^{\prime}, p\right)} \stackrel{m \rightarrow \infty}{\sim} A_{\left(n^{\prime}, n\right)\left(s^{\prime}, s\right)}^{\left(p^{\prime}, p\right)}(m+1)^{-E_{\left(n^{\prime}, n\right)\left(s^{\prime}, s\right)}^{\left(p^{\prime}, p\right)}}
$$

with $A_{\left(n^{\prime}, n\right)\left(s^{\prime}, s\right)}^{\left(p^{\prime},\right)^{\prime}} \in \mathbb{R}$, and $E_{\left(n^{\prime}, n\right)\left(s^{\prime}, s\right)}^{\left(p^{\prime}, p\right)} \geq 0$ for non-vanishing $A_{\left(n^{\prime}, n\right)\left(s^{\prime}, s\right)}^{\left(p^{\prime}, p\right)}$ by Lemma C.1 Hence each OPE-constant $C_{\left(n^{\prime}, n\right),\left(s^{\prime}, s\right)}^{\left(p^{\prime}, p\right)}$ converges to a finite limit as $m \rightarrow \infty$. In fact, the properties of basic monomial systems and (4.1.6) directly imply

\section{Lemma 4.1.4}

For the sequence $\left(\mathcal{M}_{m}, f_{m}^{j}\right)$, Conds. 1 and $3-6$ of Sect. 2.1] hold.

To meet Def. 2.1.3] we need the more general

\section{Lemma 4.1.5}

For the sequence $\left(\mathcal{M}_{m}, f_{m}^{j}\right)$, all n-point functions on $\mathbb{P}^{1}$ converge with respect to the $f_{m}^{j}$ as real analytic functions away from the partial diagonals, with the standard behaviour near the singularities (see App. A.

Proof:

By Lemma 4.1.4 all structure constants of the Virasoro algebra converge as $m \rightarrow \infty$. It will therefore suffice to prove convergence of those $n$-point functions which only contain primaries $|r, s\rangle_{m}$, since all others can be obtained from them by application of differential operators with coefficients depending polynomially on the structure constants of the Virasoro algebra. Let $V_{(r, s)}^{m}(z, \bar{z})$ denote the field which creates $|r, s\rangle_{m}$ as in A.7). By D-F1,D-F3, an $n$-point function

$$
\left\langle 0\left|V_{\left(r_{1}, s_{1}\right)}^{m}\left(z_{1} ; \bar{z}_{1}\right) \cdots V_{\left(r_{n}, s_{n}\right)}^{m}\left(z_{n} ; \bar{z}_{n}\right)\right| 0\right\rangle_{m}
$$

on $\mathbb{P}^{1}$ is a bilinear combination of a finite ( $m$-independent) number of specific conformal blocks (see 4.1.10) with coefficients given by OPE-constants. Since by Lemma 4.1.4 all OPE-constants converge as $m \rightarrow \infty$, it remains to prove that the conformal blocks converge. To this end we use their Feigin-Fuks integral representations for $\mathcal{M}_{m}$. In particular, we employ the Coulomb-gas formalism, i.e. 
a BRST construction of the $\mathcal{V}_{(r, s)}^{m}$ (see [Fe1]), which is adequate since the OPEconstants in $\mathcal{M}_{m}$ have been calculated by this technique in the first place D-F2. In fact, the correction [Fe2 to [Fe1 (3.14)] ensures that the BRST charges remain well-defined operators as $m \rightarrow \infty$, yielding the Coulomb-gas description valid in our limit.

Recall (see, e.g., Fe1 A-G-S-G] that in the Coulomb-gas formalism the $\mathcal{V}_{(r, s)}^{m}$ are obtained by a BRST construction from CHARGED FOCK SPACES, built by the action of the Heisenberg algebra on $|r, s\rangle_{m}$. In particular, primary fields of $\mathcal{M}_{m}$ are given by BRST invariant operators with screening charges, such that $U(1)$ representation theory can be used to calculate the $n$-point functions. That is, in an $n$-point function the field $V_{(r, s)}^{m}(z, \bar{z})$ can be represented in terms of products of holomorphic SCREENED VERTEX OPERATORS

$$
\begin{aligned}
V_{(r, s)}^{i, j}(z):=\oint d u_{1} \cdots \oint d u_{i} \oint d v_{1} \cdots \oint d v_{j} \\
V_{\alpha_{(r, s)}^{m}}(z) V_{\alpha_{+}^{m}}\left(u_{1}\right) \cdots V_{\alpha_{+}^{m}}\left(u_{i}\right) V_{\alpha_{-}^{m}}\left(v_{1}\right) \cdots V_{\alpha_{-}^{m}}\left(v_{j}\right)
\end{aligned}
$$

and their antiholomorphic counter-parts. Here, each $V_{\alpha}$ denotes the holomorphic part of a vertex operator of charge $\alpha$ as in Sect. 3.1 $V_{|Q ; \bar{Q}\rangle}(z, \bar{z})=V_{Q}(z) V_{\bar{Q}}(\bar{z})$, and

$$
\alpha_{ \pm}^{m}= \pm\left(\frac{m}{m+1}\right)^{ \pm 1 / 2}, \quad \alpha_{(r, s)}^{m}:=\frac{1}{2}\left((1-r) \alpha_{+}^{m}+(1-s) \alpha_{-}^{m}\right) .
$$

Each conformal block is proportional to some

$$
\begin{array}{r}
\oint_{C_{1}} d u_{1} \cdots \oint_{C_{N}} d u_{N} \oint_{S_{1}} d v_{1} \cdots \oint_{S_{M}} d v_{M}\langle 0| V_{\alpha_{1}^{m}}\left(z_{1}\right) \cdots V_{\alpha_{n}^{m}}\left(z_{n}\right) V_{-2 \alpha_{0}^{m}}(\infty)(4.1 .10) \\
V_{\alpha_{+}^{m}}\left(u_{1}\right) \cdots V_{\alpha_{+}^{m}}\left(u_{N}\right) V_{\alpha_{-}^{m}}\left(v_{1}\right) \cdots V_{\alpha_{-}^{m}}\left(v_{M}\right)|0\rangle_{\mathbb{P} 1}
\end{array}
$$

with $\alpha_{i}^{m}:=\alpha_{\left(r_{i}, s_{i}\right)}^{m}$. Here, $M$ and $N$ are determined by the $r_{i}, s_{i}$, only, such that the explicit numbers of screening charges which have to be introduced is independent of $m$. The choice of integration contours $C_{i}, S_{i} \subset \mathbb{P}^{1} \backslash\left\{z_{1}, \ldots, z_{n}\right\}$ in 4.1.10 determines the specific conformal block and is independent of $m$. This yields the description of conformal blocks by representations with screened vertex operators valid in our limit. By D-F1,D-F3 the contours can be chosen in such a way that the minimal distance between them as well as the minimal distance between the contours and the $z_{i}$ is bounded away from zero by a constant. Since the integrand of (4.1.10) is the well-known $n$-point function of vertex operators for the free bosonic theory, see (3.1.2), it therefore converges uniformly on the integration domain implying that limit and integration can be interchanged. Hence the integral of the limit function is well-defined because the integration domain is compact and does not hit singularities of the integrand.

Combining the above results, we find

\section{Proposition 4.1.6}

The sequence $\left(\mathcal{M}_{m}, f_{m}^{j}\right)$ of unitary Virasoro minimal models converges fully to a limit $\mathcal{M}_{\infty}$ according to Def. 2.1.3

Proof: 
In view of Lemmas 4.1.4 and 4.1.5 and by Def. 2.1.3 it only remains to be shown that Cond. 7 of Sect. 2.1 holds. We set

$$
\forall r, s \in \mathbb{N}-\{0\}: \quad|r, s\rangle_{\infty}:=f_{m}^{\infty}\left(|r, s\rangle_{m}\right) .
$$

By 4.1.7) we have

$$
\mathbb{H}^{\infty}=\mathcal{H}_{0,0}^{\infty}=\operatorname{span}_{\mathbb{C}}\left\{|r, r\rangle_{\infty} \mid r \in \mathbb{N}-\{0\}\right\} .
$$

Then by Lemma 2.2.1 for all $r, s^{\prime}, s \in \mathbb{N}-\{0\}$ and $h=\bar{h}=\left(s^{\prime}-s\right)^{2} / 4$, the OPEconstant $C^{\infty}\left(\psi^{*},|r, r\rangle_{\infty},\left|s^{\prime}, s\right\rangle_{\infty}\right)$ must vanish for every primary $\psi \in \mathcal{H}^{\infty}$ with $\psi \notin \widetilde{\mathbb{H}}_{h, \bar{h}}^{\infty}$. This is directly confirmed by LemmaC.1 Moreover, (C.2) implies that $C^{m}\left(\left(\left|p^{\prime}, p\right\rangle_{m}\right)^{*},|r, r\rangle_{m},\left|s^{\prime}, s\right\rangle_{m}\right)$ vanishes for all $m$ unless $\left|r-s^{(\prime)}\right|+1 \leq p^{(\prime)} \leq$ $\min \left\{r+s^{(\prime)}-1,2 m-1-r-s^{(\prime)}\right\}$ and $p^{(\prime)}+r+s^{(\prime)} \equiv 1(2)$. This restricts $p$ and $p^{\prime}$ to a finite number of possibilities as $m \rightarrow \infty$, implying Cond. 7 of Sect. 2.1 In fact, a straightforward calculation using (C.4)-(C.8) shows

$$
C_{(r, r)(s, s)}^{(p, p)}=1 \quad \text { for }|r-s|+1 \leq p \leq r+s-1, \quad p+r+s \equiv 1(2) .
$$

Therefore,

$$
\forall r, s \in \mathbb{N}-\{0\}: \quad|r, r\rangle_{\infty} ⿴|s, s\rangle_{\infty}=\sum_{\substack{p=|r-s|+1 \\ p+r+s \equiv 1(2)}}^{r+s-1}|p, p\rangle_{\infty} .
$$

Note that although we have made many choices in our construction above, the actual structure of the limit $\mathcal{M}_{\infty}$ is independent of those choices. This is largely due to the fact that Conds. 1 - 7 of Sect. 2.1] are rather restrictive. For example, recall the two basic singular vectors of levels $r s$ and $(m-r)(m+1-s)$ in the Verma module built on the primary $|r, s\rangle_{m}$. The latter state does not play a role in the limit, since its level becomes infinite as $m \rightarrow \infty$. In the language of our basic monomial systems of Def. 4.1.1 it always lies above the energy bound. On the other hand, the singular vector at level $r s$ has dimension $\frac{1}{4}(r+s)^{2}$ and implies that there also is a polynomial $P_{r s}$ of degree $r s$ such that $P_{r s}\left(L_{n}\right)|r, s\rangle_{\infty}=0$. Since up to normalization, $P_{r s}$ is uniquely determined by $r, s$, and by the structure constants of $\mathcal{V} \operatorname{ir}_{c=1}$, the dependence on the choice of the basic monomial system $\left(\mathcal{P}_{(r, s)}^{m}(N)\right)_{N \in \mathbb{N}}$ drops out in the limit.

\section{Remark 4.1.7}

In contrast to the examples discussed in Sect. 3 for the limit of $\left(\mathcal{M}_{m}, f_{m}^{j}\right)$ we obtain additional null vectors, i.e. $\mathcal{N}^{\infty} \neq\{0\}$. This is due to the fact that the conformal weights of lwvs $|r, s\rangle_{m}$ converge to $(r-s)^{2} / 4$, while the central charge converges to 1 (see (4.1.7)). By the above discussion of singular vectors, the characters of the limit Virasoro modules before quotienting out the null vectors are given by

$$
\frac{1}{\eta(q)}\left(q^{(r-s)^{2} / 4}-q^{(r+s)^{2} / 4}\right)
$$

But at $c=1$ there are null vectors $(\mathbb{B . 8})$ in the Fock spaces built on lwvs with conformal weight $h, 2 \sqrt{h} \in \mathbb{N}$, and the limit characters decompose into 
characters $(\underline{B .9})$ of irreducible representations of the Virasoro algebra of central charge $c=1$,

$$
\frac{1}{\eta(q)}\left(q^{(r-s)^{2} / 4}-q^{(r+s)^{2} / 4}\right)=\sum_{k=0}^{\min \{r, s\}-1} \chi_{\frac{1}{4}(|r-s|+2 k)^{2}} .
$$

Those submodules of $\mathcal{K}^{\infty}$, where $\mathcal{K}^{\infty} / \mathcal{N}^{\infty}=\mathcal{H}^{\infty}$ as in (2.1.3), which correspond to lwvs at positive levels consist of limit-null vectors, whose norms converge to zero for $m \rightarrow \infty$. For instance, the norm of $f_{m}^{\infty}\left(L_{1}^{m}|r, r\rangle_{m}\right), r>1$, is given by the limit of

$$
\left.\left|L_{1}^{m}\right| r, r\right\rangle\left._{m}\right|^{2}=C^{m}\left(\left(L_{1}^{m}|r, r\rangle_{m}\right)^{*}, \Omega^{m}, L_{1}^{m}|r, r\rangle_{m}\right)=2 h_{(r, r)}^{m} \stackrel{m \rightarrow \infty}{\sim} \frac{r^{2}-1}{2 m^{2}} .
$$

Thus this vector and all its descendants are elements of $\mathcal{N}^{\infty}$.

As alluded to in Rem. 2.1.5 iv the quotienting out by additional null vectors in (2.1.3) spoils the factorization properties of the limit-correlation functions on $\mathbb{P}^{1}$. However, as pointed out in [G-R-W] Sect. 3.1.1] it is possible to modify the definition of the $f_{m}^{j}$ in such a way that $\mathcal{N}^{\infty}=\{0\}$. This is achieved by SCALING UP THE ADDITIONAL NULL VECTORS. For example, we can set

$$
\tilde{f}_{m}^{j}\left(L_{1}^{m}|r, r\rangle_{m}\right):=\frac{\left.\left|L_{1}^{m}\right| r, r\right\rangle_{m} \mid}{\left.\left|L_{1}^{j}\right| r, r\right\rangle_{j} \mid} f_{m}^{j}\left(L_{1}^{m}|r, r\rangle_{m}\right) .
$$

Indeed, homomorphisms $\tilde{f}_{m}^{j}$ can be constructed in such a way that $\left(\mathcal{M}_{m}, \tilde{f}_{m}^{j}\right)$ is a sequence of CFTs with stable Virasoro algebras according to Defs. [2.1.1] 2.1.2 which does not lead to additional null vectors as $m \rightarrow \infty$. However, the modification $f_{m}^{j} \mapsto \widetilde{f}_{m}^{j}$ could destroy the convergence of correlation functions. That this is not the case, and that in fact the sequence $\left(\mathcal{M}_{m}, \widetilde{f}_{m}^{j}\right)$ of CFTs is fully convergent follows from the Coulomb-gas formalism. In the proof of Lemma 4.1.5 we have already pointed out that the expressions obtained from the Coulomb-gas formalism remain well-defined as $m \rightarrow \infty$. Recall that the Fock space representation of elements of $\mathcal{V}_{(r, s)}^{m}$ in the Coulomb-gas representation is formally obtained from an action of the positive modes of the Heisenberg algebra on $|r, s\rangle_{m}$. Hence singular vectors with respect to the action of $\mathcal{V}_{i_{c_{m}}}$ on $|r, s\rangle_{m}$ are automatically zero, see Ex. 4.1.8 for an illustration. Namely, each singular vector $\nu \in \mathcal{K}^{\infty}$ is of the form $\nu=f_{m}^{\infty}\left(\nu^{m}\right)$ with $\nu^{m}=Q^{m}|r, s\rangle_{m}$, where $Q^{m}$ is an operator on $\mathcal{H}^{m}$ which can formally be written as polynomial in the positive modes of the Heisenberg algebra, with each coefficient converging to zero as $m \rightarrow \infty$. In fact, each coefficient is a power series in $\frac{1}{m}$ with vanishing constant term. Therefore, our rescaling yields $\widetilde{\nu}=\widetilde{f}_{m}^{\infty}\left(\nu^{m}\right)=\widetilde{Q}|r, s\rangle_{\infty}$ with an operator $\widetilde{Q}$ on $\mathcal{H}^{\infty}$ which again can formally be obtained as polynomial in the positive modes of the Heisenberg algebra. Hence all correlation functions involving $\widetilde{Q}|r, s\rangle_{\infty}$ also converge.

This way, we can obtain a limit of the A-series of Virasoro minimal models whose correlation functions on $\mathbb{P}^{1}$ have the usual factorization properties. As a model case, in Lemma C.2 we also show by direct calculation that no divergences are introduced in $C\left(\left|p^{\prime}, p\right\rangle_{\infty}^{*}, L_{1}|r, r\rangle_{\infty},\left|s^{\prime}, s\right\rangle_{\infty}\right)$ when the singular vectors $L_{1}|r, r\rangle_{\infty}$ are scaled up. 


\section{Example 4.1.8}

As in Ex. 1.2.2 let $\mathcal{C}_{R_{i}}, i \in \mathbb{N}$, denote the CFT with central charge $c=1$ that describes a boson compactified on a circle of radius $R_{i}$, here with $R_{i}:=1+\frac{\sqrt{2}}{i}$. See in particular (1.2.9) for notations. According to (B.7) - B.9 the Verma module built on each $\left|Q_{m, n}^{i} ; \bar{Q}_{m, n}^{i}\right\rangle_{i}, m, n \in \mathbb{Z}$, by the action of the Virasoro algebra is irreducible if $(m, n) \neq(0,0)$ because all our $R_{i}^{2}$ are irrational. We can therefore define a direct system $\left(\mathcal{H}^{i}, \widehat{f}_{i}^{j}\right)$ by

$$
\forall(m, n) \neq(0,0): \quad \widehat{f}_{i}^{j}\left(P\left(L_{k}^{i} ; \bar{L}_{\bar{k}}^{i}\right)\left|Q_{m, n}^{i} ; \bar{Q}_{m, n}^{i}\right\rangle_{i}\right):=P\left(L_{k}^{j} ; \bar{L}_{\bar{k}}^{j}\right)\left|Q_{m, n}^{j} ; \bar{Q}_{m, n}^{j}\right\rangle_{j},
$$

where $P$ denotes a polynomial in the $L_{k}, \bar{L}_{\bar{k}}, k, \bar{k}>0$. In the vacuum sector we use

$$
\widehat{f}_{i}^{j}\left(P\left(a_{k}^{i} ; \bar{a}_{\bar{k}}^{i}\right)|0 ; 0\rangle_{i}\right):=P\left(a_{k}^{j} ; \bar{a}_{\bar{k}}^{j}\right)|0 ; 0\rangle_{j}
$$

as in (3.1.5), where as usual $a_{k}^{i}, \bar{a}_{\bar{k}}^{i}$ denote the modes of the generators $j, \bar{\jmath}$ of $\mathfrak{u}(1) \oplus \overline{\mathfrak{u}(1)}$ in $\mathcal{C}_{R_{i}}$. One checks that this gives a convergent sequence $\left(\mathcal{H}^{i}, \widehat{f}_{i}^{j}\right)$ of CFTs, but the direct limit $\widehat{\mathcal{K}}^{\infty}$ possesses null vectors in $\widehat{\mathcal{N}}^{\infty}$, where $\widehat{\mathcal{H}}^{\infty}=$ $\widehat{\mathcal{K}}^{\infty} / \widehat{\mathcal{N}}^{\infty}$. For example,

$$
\nu^{i}:=\left(L_{2}^{i}-\left(L_{1}^{i}\right)^{2}\right)\left|Q_{1,0}^{i} ; \bar{Q}_{1,0}^{i}\right\rangle_{i}
$$

where for $\left|Q_{1,0}^{i} ; \bar{Q}_{1,0}^{i}\right\rangle_{i}$ we have $Q_{1,0}^{i}=\bar{Q}_{1,0}^{i}=\frac{1}{\sqrt{2} R_{i}} \stackrel{i \rightarrow \infty}{\longrightarrow} \frac{1}{\sqrt{2}}, h_{i}=\bar{h}_{i}=$ $\frac{1}{4\left(R_{i}\right)^{2}} \stackrel{i \rightarrow \infty}{\longrightarrow} \frac{1}{4}$, which gives a null vector $\nu=f_{i}^{\infty}\left(\nu^{i}\right)$.

On the other hand, in Sect. 3.1 we have already constructed a fully convergent sequence $\left(\mathcal{H}^{i}, f_{i}^{j}\right)$ of CFTs via

$$
f_{i}^{j}\left(P\left(a_{k}^{i} ; \bar{a}_{\bar{k}}^{i}\right)\left|Q_{m, n}^{i} ; \bar{Q}_{m, n}^{i}\right\rangle_{i}\right):=P\left(a_{k}^{j} ; \bar{a}_{\bar{k}}^{j}\right)\left|Q_{m, n}^{j} ; \bar{Q}_{m, n}^{j}\right\rangle_{j}
$$

with $P$ as above. Now the limit is the $\mathfrak{s u}(2)_{1}$ WZW model, i.e. a full fledged well-defined CFT. Note that in terms of the latter Fock space representation, $\nu^{i}$ in (4.1.14) is given by

$$
\nu^{i}=\frac{1}{2}\left(a_{1}^{i}\right)^{2}\left(1-2\left(a_{0}^{i}\right)^{2}\right)\left|Q_{1,0}^{i} ; \bar{Q}_{1,0}^{i}\right\rangle_{i}=\frac{1}{2}\left(a_{1}^{i}\right)^{2}\left(1-\left(R_{i}\right)^{-2}\right)\left|Q_{1,0}^{i} ; \bar{Q}_{1,0}^{i}\right\rangle_{i} .
$$

Hence $f_{i}^{\infty}\left(\nu^{i}\right) \stackrel{i \rightarrow \infty}{\longrightarrow} 0$ in $\mathcal{H}^{\infty}=\mathcal{K}^{\infty}$. The direct system $\left(\mathcal{H}^{i}, f_{i}^{j}\right)$ yields the vectors $\left(a_{1}\right)^{2}\left|\frac{1}{\sqrt{2}} ; \frac{1}{\sqrt{2}}\right\rangle_{\infty}, a_{2}\left|\frac{1}{\sqrt{2}} ; \frac{1}{\sqrt{2}}\right\rangle_{\infty}$ as linearly independent elements of $\mathcal{H}_{2+\frac{1}{4}, \frac{1}{4}}^{\infty}$, where the combination $\left(a_{2}-\sqrt{2}\left(a_{1}\right)^{2}\right)\left|\frac{1}{\sqrt{2}} ; \frac{1}{\sqrt{2}}\right\rangle_{\infty}$ is Virasoro primary. For $\left(\mathcal{H}^{i}, \widehat{f}_{i}^{j}\right)$, the corresponding vectors $\left(L_{1}\right)^{2}\left|\frac{1}{\sqrt{2}} ; \frac{1}{\sqrt{2}}\right\rangle_{\infty}, L_{2}\left|\frac{1}{\sqrt{2}} ; \frac{1}{\sqrt{2}}\right\rangle_{\infty}$ differ by the null vector $\nu$ and are thus identified in $\widehat{\mathcal{H}}_{2+\frac{1}{4}, \frac{1}{4}}^{\infty}$. However, the above directly implies how the $\widehat{f}_{i}^{j}$ can be redefined by scaling up the additional null vectors, and then both limits give the same well-defined CFT.

To approach the full limit structure obtained on the pre-Hilbert space $\mathcal{H}^{\infty}$, recall that in the proof of Lemma 4.1.5 and in Rem. 4.1.7 we have argued that the correlation functions in $\mathcal{M}_{\infty}$ are adequately described in terms of 
the Coulomb-gas formalism. A closer study of this formalism also shows that it should be possible to represent the operator product algebra of the limit within the $\mathfrak{s u}(2)_{1}$ WZW model ${ }^{17}$. Namely, as follows from performing the limit in (4.1.9), the operator corresponding to $|r, s\rangle_{\infty}$ in a given correlation function should be represented by a combination of the left-right symmetric $\mathfrak{u}(1)$ vertex operator $V_{\left|Q=\frac{r-s}{\sqrt{2}} ; \bar{Q}=\frac{r-s}{\sqrt{2}}\right\rangle}(z, \bar{z})$ of the circle model $\mathcal{C}_{R=1}$ and the zero modes $Q_{ \pm}$ of the holomorphic fields $J_{ \pm}(z)$ which create $|Q ; \bar{Q}\rangle=| \pm \sqrt{2} ; 0\rangle$ as in (B.10), along with their antiholomorphic counterparts.

4.2. Geometric interpretation of $\mathcal{M}(m, m+1)_{m \rightarrow \infty}$. Note that by (4.1.7) the limit $\mathcal{M}_{\infty}$ of the sequence of unitary Virasoro minimal models has an infinite degeneracy of every energy level. This means that we cannot interpret $\mathcal{M}_{\infty}$ as part of a well-defined CFT. However, the degeneration of the vacuum sector allows us to apply the techniques introduced in Sect.2.2 and to find a geometric interpretation of the limit. Indeed, in Prop. 4.2.2 below we identify the algebra $\mathcal{A}^{\infty}$ obtained from $\mathbb{H}^{\infty}$ by 4.1.12) with the algebra generated by the CHEBYSHEV POLYNOMIALS OF THE SECOND KIND, i.e. with the algebra of continuous functions on an interval:

\section{Lemma 4.2.1}

For every $r \in \mathbb{N}-\{0\}$, let $U_{r}$ denote the $r^{\text {th }}$ Chebyshev polynomial of the SECOND KIND:

$$
U_{r}(\cos x):=\frac{\sin (x r)}{\sin x}, \quad x \in[0, \pi] .
$$

Then $U_{r}(t=\cos x)$ is a polynomial of degree $r-1$ in $t \in[-1,1]$, and the $U_{r}(t)$ form an orthonormal system of polynomials with respect to the scalar product

$$
\langle f, g\rangle_{\omega}:=\int_{-1}^{1} \overline{f(t)} g(t) \omega(t) d t, \quad \omega(t):=\frac{2}{\pi} \sqrt{1-t^{2}} .
$$

Moreover, the Chebyshev polynomials of the second kind obey the recursion relation

$$
\forall r, s \in \mathbb{N}-\{0\}, \forall t \in[-1,1]: \quad U_{r}(t) U_{s}(t)=\sum_{\substack{p=|r-s|+1, p+r+s \equiv 1(2)}}^{r+s-1} U_{p}(t) .
$$

The proof of Lemma 4.2.1 is a straightforward calculation, see e.g. He Problems 3.1.10(a)]. Note in particular that this lemma implies

$$
\forall t \in[-1,1], \forall x \in[0, \pi]: \quad \delta_{\cos x}(t)=\sum_{p^{\prime}=1}^{\infty} U_{p^{\prime}}(t) U_{p^{\prime}}(\cos x) \frac{2}{\pi} \sin x .
$$

We are now in the position to give a geometric interpretation for our limit according to Def. 2.2.6

\footnotetext{
17 This is in accord with $\left[\mathrm{D}-\mathrm{V}-\mathrm{V}\right.$ p. 655], where it is stated that the $\mathfrak{s u}(2)_{1}$ WZW model "in some sense can be regarded as the limit $c \rightarrow 1$ of the discrete unitary series".
} 


\section{Proposition 4.2.2}

The limit $\mathcal{M}_{\infty}$ of the sequence $\left(\mathcal{M}_{m}, f_{m}^{j}\right)$ of unitary Virasoro minimal models has a geometric interpretation on the interval $[0, \pi]$ equipped with the dilatoncorrected metric $g(x)=\frac{4}{\pi^{2}} \sin ^{4} x$ and dilaton $\Phi$ such that $e^{2 \Phi(x)}=\frac{2}{\pi} \sin ^{2} x$ for $x \in[0, \pi]$.

\section{Proof:}

As a first step, we need to construct a spectral pre-triple $\left(\mathbb{H}^{\infty}, H^{\infty}, \mathcal{A}^{\infty}\right)$ from our limit $\mathcal{M}_{\infty}$ according to Def. 2.2.6. In fact, by Def. 2.2.2 $\mathbb{H}^{\infty}$ is given in (4.1.11), and $\mathcal{A}^{\infty}$ is the associated zero-mode algebra specified in (4.1.12). Moreover, (4.1.3) shows that on $\mathbb{H}^{\infty}$, according to Def. 2.2.6 we need to set

$$
\begin{aligned}
\forall r \in \mathbb{N}-\{0\}: \quad \lambda_{r}^{2}:=\lim _{m \rightarrow \infty} m^{2}\left(h_{(r, r)}^{m}+\bar{h}_{(r, r)}^{m}\right) & =\frac{r^{2}-1}{2}, \\
H^{\infty}|r, r\rangle_{\infty} & :=\frac{r^{2}-1}{2}|r, r\rangle_{\infty} .
\end{aligned}
$$

Comparison of (4.1.12) with 4.2.3 shows that $\mathcal{A}^{\infty}$ agrees with the algebra generated by the Chebyshev polynomials of the second kind. Here, similarly to the discussion of Chebyshev polynomials of the first kind at the end of Sect. 3.2] we view the $U_{r}$ as functions $x \mapsto U_{r}(\cos x)$ with $x \in[0, \pi]$. Therefore, $\overline{\mathcal{A}^{\infty}}$ can be identified with the algebra of smooth functions on $[0, \pi]$, and (4.2.2) shows that $[0, \pi]$ is equipped with the dilaton-corrected metric $g$ with $\operatorname{dvol}_{g}=\sqrt{g(x)} d x=$ $\frac{2}{\pi} \sin ^{2} x d x$ as claimed. By the discussion in Sect. 1.1 it therefore remains to identify $H^{\infty}$ in (4.2.5) with the generalized Laplacian $H$ as defined in (1.1.6) and to read off the dilaton $\Phi$. To this end we use the characterization (1.1.5), that is, for all $f, h \in C^{\infty}([0, \pi])$ we must have $\langle f, 2 H h\rangle_{\omega}=\left\langle f^{\prime}, h^{\prime}\right\rangle_{\omega}$. Since

$$
\begin{aligned}
\langle f, 2 H h\rangle_{\omega} \stackrel{!}{=}\left\langle f^{\prime}, h^{\prime}\right\rangle_{\omega} \stackrel{\text { 4.2.2 }}{=} \int_{0}^{\pi} \overline{f^{\prime}(x)} h^{\prime}(x) \frac{2}{\pi} \sin ^{2} x d x \\
=-\int_{0}^{\pi} \overline{f(x)} \frac{d}{d x}\left(\sin ^{2} x h^{\prime}(x)\right) \frac{2}{\pi} d x,
\end{aligned}
$$

we deduce that $2 H=-\sin ^{-2} x \frac{d}{d x} \sin ^{2} x \frac{d}{d x}$, and thus $\widetilde{g}(x) \equiv 1$ and $e^{2 \Phi(x)}=$ $\frac{2}{\pi} \sin ^{2} x$. With (4.2.1) one now checks that $H U_{r}(\cos x)=\frac{r^{2}-1}{2} U_{r}(\cos x)$, in perfect agreement with 4.2.5.

\section{Remark 4.2.3}

The distance functional, which is associated to the dilaton-corrected metric $g(x)=\frac{4}{\pi^{2}} \sin ^{4} x$ on the interval $[0, \pi]$ determined in Prop. 4.2.2, is

$$
\forall a, b \in[0, \pi]: \quad d(a, b)=\frac{1}{2 \pi}|\xi(a)-\xi(b)| \quad \text { with } \quad \xi(\tau):=2 \tau-\sin (2 \tau) .
$$

Here, $\xi(\tau / 2)$ is the $x$-coordinate of a regular cycloid in Cartesian coordinates. That is, if we consider a unit wheel which roles horizontally at unit speed, then $2 \pi d(0, \tau / 2)$ measures the distance that the point $(2,0)$ on the wheel travels horizontally within the time $\tau$.

\section{Remark 4.2.4}

On the level of topological manifolds, our geometric interpretation of $\mathcal{M}_{\infty}$ on an interval could have been predicted from the discussion in F-G §3.3]. Namely, 
the unitary Virasoro minimal model $\mathcal{M}_{m}$ can be obtained by an $\mathfrak{s u}(2)$-coset construction:

$$
\mathcal{M}_{m} \longleftrightarrow \frac{\mathfrak{s u}(2)_{m-2} \oplus \mathfrak{s u}(2)_{1}}{\mathfrak{s u}(2)_{m-1}}
$$

In this language, the labels $r$ and $s$ in $|r, s\rangle_{m}$ refer to the relevant representations of $\mathfrak{s u}(2)_{m-2}$ and $\mathfrak{s u}(2)_{m-1}$, respectively. Loosely speaking, since only states with $r=s$ enter in our zero-mode algebra, our geometric interpretation can be expected to yield a semiclassical limit of the coset WZW model $\mathfrak{s u}(2)_{m} / \mathfrak{s u}(2)_{m}$ as $m \rightarrow \infty$. That is, by [F-G] (3.25)-(3.26)] the limit should have a geometric interpretation on the space $\mathrm{SU}(2) / \mathrm{Ad}(\mathrm{SU}(2)) \simeq T / W$ with $T$ the Cartan subgroup and $W$ the Weyl group of $\mathrm{SU}(2)$. Indeed, with $T=U(1), W=\mathbb{Z}_{2}$ we obtain $T / W \simeq[0, \pi]$. An analogous observation was already made in $\underline{\mathrm{R}-\mathrm{W} 2}$. There, it was also pointed out that ${ }^{18}$ the geometric interpretation of $\mathcal{M}_{\infty}$ on the interval fits nicely with an analysis of the qualitative Landau-Ginzburg description for the minimal models $\mathcal{M}_{m}$ [Za1]: As $m \rightarrow \infty$, the Landau-Ginzburg potential approaches a square well with walls at $X= \pm 1$, forcing the scalar field $X$ of the Landau-Ginzburg theory to take values on the interval $[-1,1]$.

The sigma model metric, in principle, could also be obtained by a gauged WZW model construction as was done in [M-M-S] in the case of $\mathfrak{s u}(2)_{k} / \mathfrak{u}(1)$.

\section{Remark 4.2.5}

Apart from the direct limit construction studied above, one can introduce other sensible limits for the family $\mathcal{M}_{m}$ as $m \rightarrow \infty$, similarly to Rem. 3.1.1 In particular, if there is a system of epimorphisms $f_{m}: \widetilde{\mathcal{H}}^{\infty} \longrightarrow \mathcal{H}^{m}$ such that all limits

$$
\left\langle 0\left|\varphi_{1}\left(z_{1}, \bar{z}_{1}\right) \ldots \varphi_{n}\left(z_{n}, \bar{z}_{n}\right)\right| 0\right\rangle:=\lim _{m \rightarrow \infty}\left\langle 0\left|f_{m}\left(\varphi_{1}\right)\left(z_{1}, \bar{z}_{1}\right) \ldots f_{m}\left(\varphi_{n}\right)\left(z_{n}, \bar{z}_{n}\right)\right| 0\right\rangle_{m}
$$

of $n$-point functions exist, then $\widetilde{\mathcal{H}}^{\infty}$ can be interpreted as pre-Hilbert space of a limit theory $M_{\infty}$. We believe that this is the structure underlying the ideas of $\mathrm{G}-\mathrm{R}-\mathrm{W}] \mathrm{R}-\mathrm{W} 1 \mathrm{R}-\mathrm{W} 2$. Indeed, there the authors find a limiting pre-Hilbert space of the form

$$
\widetilde{\mathcal{H}}^{\infty}=\bigoplus_{r \in \mathbb{R}^{+}-\mathbb{N}} \mathcal{V}_{r^{2} / 4}^{\text {gen }},
$$

where for $h \in \mathbb{R}^{+}$with $2 \sqrt{h} \notin \mathbb{N}, \mathcal{V}_{h}^{\text {gen }}$ denotes the generic representation of the Virasoro algebra $\mathcal{V i r}_{c=1}$ with character (B.7). Analogously to the situation in Rem. 3.1.1 no degeneration phenomena occur in this procedure, and the limit $M_{\infty}$ is conjectured to be part of a well-defined non-rational CFT with central charge $c=1$, which has an interesting resemblance to Liouville theory. Evidence for this conjecture is given in [R-W1], where in particular crossing symmetry is proven in some model cases.

It seems that the two limits $\mathcal{M}_{\infty}$ and $M_{\infty}$ are complementary in many respects: For instance, the representation content of $\widetilde{\mathcal{H}}^{\infty}$ is complementary to the one we have found in $\mathcal{H}^{\infty}$, see (4.1.7). Moreover, while the limit $M_{\infty}$ seems to be a well-defined CFT, $\mathcal{M}_{\infty}$ shows the degeneration phenomena discussed above, which allow to extract a geometric interpretation from the limit structures.

\footnotetext{
18 according to J. Cardy
} 
A third approach to limiting processes is taken in [Fu-S. There, limits of WZW models at infinite level are introduced by means of INVERSE LIMITS instead of direct limits. While our direct limit construction takes advantage of those structures which the pre-Hilbert spaces of minimal models $\mathcal{M}_{m}$ share at $m \gg 0$ and for sufficiently low conformal dimensions, the inverse limit construction of [Fu-S allows to interpret the collection of fusion rings of $\mathfrak{g}-\mathrm{WZW}$ models as a category and to identify a projective system in it. Clearly, as mentioned above, we cannot view the family $\left(\mathcal{M}_{m}\right)_{m \in \mathbb{N}-\{0,1\}}$ of minimal models as direct system of CFTs with the natural ordering induced by $\mathbb{N}$. The same is true already on the level of $\mathfrak{g}-\mathrm{WZW}$ models; however, in [Fu-S] a suitable non-standard partial ordering is found for the latter. Whether geometric interpretations of $\left(\mathcal{M}_{m}\right)_{m \rightarrow \infty}$ with the expected properties arise from this construction remains to be seen.

We have not worked out the details of an application of our techniques to $\mathfrak{g}$-WZW models at infinite level. However, we expect that the results of [F-G] should tie in naturally thus leading to a direct limit construction with the expected geometric interpretation on the group manifold $G$.

The results of Prop. 4.2.2 and Rem.4.2.4 imply that under the coordinate change $t=\cos x$, our limit $\mathcal{M}_{\infty}$ has a geometric interpretation on the unit interval. By the ideas of [F-G] this also means that each unitary Virasoro minimal model $\mathcal{M}_{m}$ with $m \gg 0$ can be regarded as sigma model on the unit interval. We therefore expect to gain some insight ${ }^{19}$ into the shape of the D-branes in this bulk-geometry by considering the bulk-boundary couplings for $m \gg 0$.

Recall that for each $\mathcal{M}_{m}$ we use the diagonal, that is the charge conjugation invariant partition function. Hence the Ishibashi states $\left.\left|p^{\prime}, p\right\rangle\right\rangle_{m}$ are labeled by $\left(p^{\prime}, p\right) \in \mathcal{N}_{m}$ with $\mathcal{N}_{m}$ as in (4.1.2). Moreover, each $(r, s) \in \mathcal{N}_{m}$ labels a boundary condition. Its bulk-boundary coupling with respect to $\left.\left|p^{\prime}, p\right\rangle\right\rangle_{m}$ is given by

$$
\begin{aligned}
B_{(r, s)}^{\left(p^{\prime}, p\right)} & =\frac{S_{(r, s)\left(p^{\prime}, p\right)}}{\sqrt{S_{(1,1)\left(p^{\prime}, p\right)}}} \\
& =(-1)^{(r+s)\left(p^{\prime}+p\right)}\left(\frac{8}{m(m+1)}\right)^{\frac{1}{4}} \frac{\sin \left(\frac{\pi r p^{\prime}}{m}\right) \sin \left(\frac{\pi s p}{m+1}\right)}{\sqrt{\sin \left(\frac{\pi p^{\prime}}{m}\right) \sin \left(\frac{\pi p}{m+1}\right)}} .
\end{aligned}
$$

In order to investigate the geometry of the D-branes, we can restrict to the couplings of the bulk-fields $\left(p^{\prime}, p^{\prime}\right)$ which by Prop. 4.2.2 correspond to the Chebyshev polynomials $U_{p^{\prime}}$ of the second kind. This means that we will focus on the bulkboundary couplings $B_{(r, s)}^{\left(p^{\prime}, p^{\prime}\right)}$ and the bulk-boundary coupling support functions

$$
f_{(r, s)}^{m}(t):=\frac{2}{\pi}\left(\frac{8}{m(m+1)}\right)^{-\frac{1}{4}} \sum_{p^{\prime}=1}^{m-1} U_{p^{\prime}}(t) B_{(r, s)}^{\left(p^{\prime}, p^{\prime}\right)} .
$$

In the above definition of $f_{(r, s)}^{m}$ we have introduced the appropriate pre-factor corresponding to the rescaling in 4.2.5) by hand. In order to analyze $f_{(r, s)}^{m}(t)$ for $m \gg 0$, we use $t=\cos x$ as before, and divide the domain of definition of $x$, the interval $[0, \pi]$, equidistantly. That is, we set

$$
\forall(r, s) \in \mathcal{N}_{m}: \quad x_{r}:=\frac{r \pi}{m}, \quad \widetilde{x}_{s}:=\frac{s \pi}{m+1} .
$$

19 strictly speaking, after extending our constructions of Sect. 2 to the boundary sector 
Note the following useful reformulation of (4.2.1) for all $p, r \in \mathbb{N}-\{0\}$ :

$$
U_{r}\left(\cos \left(x_{p}\right)\right) \sin \left(x_{p}\right)=\sin \left(r x_{p}\right)=\sin \left(p x_{r}\right)=U_{p}\left(\cos \left(x_{r}\right)\right) \sin \left(x_{r}\right),
$$

and analogously for $\widetilde{x}_{p}, \widetilde{x}_{r}$. Using $x_{r} \approx \widetilde{x}_{r}$ for $m \gg 0$, we therefore find:

$$
\begin{aligned}
& f_{(r, s)}^{m}(t) \stackrel{[4.2 .6}{=} \frac{2}{\pi} \sum_{p^{\prime}=1}^{m-1} U_{p^{\prime}}(t) \frac{\sin \left(r x_{p^{\prime}}\right) \sin \left(s \widetilde{x}_{p^{\prime}}\right)}{\sqrt{\sin \left(x_{p^{\prime}}\right) \sin \left(\widetilde{x}_{p^{\prime}}\right)}} \\
& \stackrel{4.2 .7}{=} \frac{2}{\pi} \sum_{p^{\prime}=1}^{m-1} U_{p^{\prime}}(t) U_{r}\left(\cos \left(x_{p^{\prime}}\right)\right) U_{s}\left(\cos \left(\widetilde{x}_{p^{\prime}}\right)\right) \sqrt{\sin \left(x_{p^{\prime}}\right) \sin \left(\widetilde{x}_{p^{\prime}}\right)} \\
& \stackrel{m \rightarrow \infty}{\sim} \frac{2}{\pi} \sum_{p^{\prime}=1}^{\infty} U_{p^{\prime}}(t) U_{r}\left(\cos \left(x_{p^{\prime}}\right)\right) U_{s}\left(\cos \left(x_{p^{\prime}}\right)\right) \sin \left(x_{p^{\prime}}\right) \\
& \stackrel{4.2 .3}{=} \sum_{\substack{p=|r-s|+1, p+r+s \equiv 1(2)}}^{r+s-1} \frac{2}{\pi} \sum_{p^{\prime}=1}^{\infty} U_{p^{\prime}}(t) U_{p}\left(\cos \left(x_{p^{\prime}}\right)\right) \sin \left(x_{p^{\prime}}\right) \\
& \stackrel{4.2 .7}{=} \sum_{\substack{p=|r-s|+1, p+r+s \equiv 1(2)}}^{r+s-1} \frac{2}{\pi} \sum_{p^{\prime}=1}^{\infty} U_{p^{\prime}}(t) U_{p^{\prime}}\left(\cos \left(x_{p}\right)\right) \sin \left(x_{p}\right) \\
& \text { (4.2.4) } \sum_{\substack{p=|r-s|+1, p+r+s \equiv 1(2)}}^{r+s-1} \delta_{\cos \left(x_{p}\right)}(t) \text {. }
\end{aligned}
$$

We interpret this calculation in form of

\section{Remark 4.2.6}

For the unitary Virasoro minimal models $\mathcal{M}_{m}$ at $m \gg 0$, the D-branes corresponding to stable boundary states labeled by $(r, 1)$ which are elementary in the sense of [R-R-S] and the D-branes corresponding to the unstable boundary states $(1, s)$ can be interpreted as being localized in the points $t=\cos \left(x_{r}\right)=\cos \left(\frac{\pi r}{m}\right)$ and $t=\cos \left(\widetilde{x}_{s}\right)=\cos \left(\frac{\pi s}{m+1}\right)$ on the interval $[-1,1]$, respectively. On the other hand, D-branes corresponding to the unstable boundary states $(r, s)$ with $r \neq 1$, $s \neq 1$ are supported on a union of these points. In view of Rem. 4.2.4 this is in accord with the general shape of D-branes in coset models Gaw $\mathrm{Fr}-\mathrm{S}$.

\section{Discussion}

To conclude, let us address some open questions arising from our investigations. Of course, there are several interesting unsolved problems concerning the degenerating limit $\mathcal{M}_{\infty}$ of the A-series of unitary Virasoro minimal models of Sect. [4 For example, it would be interesting to gain more insight into the representation of this limit within the $\mathfrak{s u}(2)_{1}$ WZW model, as mentioned at the end of Sect.4.1 In particular, there are two fusion closed subsectors in $\mathcal{M}_{\infty}$, corresponding to the states $|r, 1\rangle_{\infty}, r \in \mathbb{N}-\{0\}$, and $|1, s\rangle_{\infty}, s \in \mathbb{N}-\{0\}$, respectively. We expect 
them to have a comparatively simple description in terms of the $\mathfrak{s u}(2)_{1}$ WZW model, because no additional null vectors occur in the corresponding Verma modules. Moreover, by acting with the zero mode algebra $\mathcal{A}^{\infty}$ on one of these subsectors, one can generate the entire limit pre-Hilbert space $\mathcal{H}^{\infty}$. Thus an understanding of these subsectors should also allow some insight into the geometry of the entire $\mathcal{A}^{\infty}$ module $\mathcal{H}^{\infty}$, for instance the fiber structure of the corresponding sheaf. Finally, one could try to extract the non-commutative geometries from the Virasoro minimal models at finite level which at infinite level reduce to the limit geometry on the interval determined in Prop.4.2.2

Next, a generalization of our discussion in Sect. 4 to WZW models and their cosets in general would be nice, e.g. to the families of unitary super-Virasoro minimal models.

More generally, for all limits of degenerating sequences of CFTs, it would be interesting to understand the compatibility of the limit structures with the action of the zero mode algebra $\mathcal{A}^{\infty}$. In particular, the limit OPE-constants are $\mathcal{A}^{\infty}$ homogeneous and therefore should be induced by a corresponding fiberwise structure on the sheaf with $\mathcal{H}^{\infty}$ as space of sections. It is likely that the entire limit can be understood in terms of such fiberwise structures together with the $\mathcal{A}^{\infty}$ action. This is in accord with the results of $\mathrm{K}-\mathrm{S}$.

In fact, the zero mode algebra would be an interesting object to study in its own right, not least because there seems to be a relation to Zhu's algebra as mentioned in Sect. 1.2.

Finally, it would be natural to extend our constructions to the boundary sector. This could allow a more conceptual understanding of geometric interpretations of D-branes, for example in terms of the K-theory of $\mathcal{A}^{\infty}$.

\section{A. Properties of conformal field theories}

In this Appendix, we collect some properties of CFTs that are used in the main text. Recall the Virasoro algebra $\mathcal{V} \operatorname{ir}_{c}$ at central charge $c$, with generators $L_{n}, n \in$ $\mathbb{Z}$

$$
\forall m, n \in \mathbb{Z}: \quad\left[L_{m}, L_{n}\right]=(n-m) L_{m+n}+\frac{c}{12}\left(n^{3}-n\right) \delta_{m+n, 0} .
$$

In a given $\mathrm{CFT} \mathcal{C}=(\mathcal{H}, *, \Omega, T, \bar{T}, C)$, the vacuum $\Omega \in \mathcal{H}$ and its dual $\Omega^{*} \in \check{\mathcal{H}}^{*}$ are characterized by

$$
*(\Omega)=\Omega ; \quad \Omega^{*}(\Omega)=1 ; \quad \forall n \leq 1: L_{n} \Omega=\bar{L}_{n} \Omega=0 ; \quad L_{n}^{\dagger}\left(\Omega^{*}\right)=\bar{L}_{n}^{\dagger}\left(\Omega^{*}\right)=0 .
$$

The map $\mathcal{H} \rightarrow \check{\mathcal{H}}^{*}, \psi \mapsto \psi^{*}$ of (1.2.4) can be explained by the relation between our OPE-coefficients $C$ and the $n$-POINT FUNCTIONS

$$
\mathcal{H}^{\otimes n} \ni \varphi_{1} \otimes \cdots \otimes \varphi_{n} \quad \longmapsto \quad\left\langle 0\left|\varphi_{1}\left(z_{1}, \bar{z}_{1}\right) \ldots \varphi_{n}\left(z_{n}, \bar{z}_{n}\right)\right| 0\right\rangle_{\Sigma}
$$

of a CFT. Here, $\Sigma$ is a conformal surface, and the right hand side of A.3) denotes a real analytic function $\Sigma^{n} \backslash D \rightarrow \mathbb{C}$ outside the partial diagonals $D=\cup_{i, j} D_{i, j}$ with $D_{i, j}:=\left\{\left(z_{1}, \ldots, z_{n}\right) \in \Sigma^{n} \mid z_{i}=z_{j}\right\}$. Moreover, the right hand side of A.3) possesses expansions around the partial diagonals $D_{i, j}$ :

$$
\sum_{(r, \bar{r}) \in R_{i, j}} a_{r \bar{r}}\left(z_{1}, \bar{z}_{1} ; \ldots ; z_{i-1}, \bar{z}_{i-1} ; z_{i+1}, \bar{z}_{i+1} ; \ldots ; z_{n}, \bar{z}_{n}\right)\left(z_{i}-z_{j}\right)^{r}\left(\bar{z}_{i}-\bar{z}_{j}\right)^{\bar{T}} .
$$


Here, $R_{i, j} \subset \mathbb{R}^{2}$ is countable, and only finitely many $a_{r \bar{r}}$ are non-zero for $r<0$ or $\bar{r}<0$. Furthermore, the $a_{r \bar{r}}$ themselves are linear combinations of $(n-1)$ point functions with OPE-coefficients as linear factors. Finally, the right hand side of A.3. is invariant under permutation of the $\varphi_{i}\left(z_{i}, \bar{z}_{i}\right)$. One says that the correlation functions constitute a REPRESENTATION OF THE OPE.

It is a basic feature of CFTs that each state $\psi \in \mathcal{H}$ possesses an ADJOINT $\psi^{\dagger} \in \mathcal{H}$ such that two-point functions on the sphere $\Sigma=\mathbb{C} \cup\{\infty\}=\mathbb{P}^{1}$ encode the metric on $\mathcal{H}$ :

$$
\forall \chi, \psi \in \mathcal{H}: \quad\langle\psi \mid \chi\rangle=\lim _{w, \zeta \rightarrow 0}\left\langle 0\left|\psi^{\dagger}\left(\bar{w}^{-1}, w^{-1}\right) \chi(\zeta, \bar{\zeta})\right| 0\right\rangle_{\mathbb{P}^{1}} .
$$

Using conformal invariance one can determine $\psi^{\dagger}(z, \bar{z})$ as the image of $* \psi(z, \bar{z})$ under the transformation $f: z \mapsto 1 / z, \bar{z} \mapsto 1 / \bar{z}$. In particular, if $\varphi \in \mathcal{H}_{h, \bar{h}}$ is real and QUASI-PRIMARY (e.g. $\varphi=T$ ), then we can write

$$
\varphi^{\dagger}(z, \bar{z})=\varphi\left(\bar{z}^{-1}, z^{-1}\right) \bar{z}^{-2 h} z^{-2 \bar{h}}
$$

As an abbreviation, one defines IN- AND OUT-STATES by setting

$$
\begin{aligned}
\forall \chi, \psi \in \mathcal{H}: \quad\langle\psi|:=\lim _{w \rightarrow 0}\langle 0| \psi^{\dagger}\left(\bar{w}^{-1}, w^{-1}\right), \\
|\chi\rangle:=\lim _{\zeta \rightarrow 0} \chi(\zeta, \bar{\zeta})|0\rangle_{\mathbb{P}^{1}} .
\end{aligned}
$$

Now the OPE-coefficients $C$ can be recovered as

$$
\begin{aligned}
\forall \varphi, \chi, \psi \in \mathcal{H}: \quad C\left(\psi^{*}, \varphi, \chi\right) & =\langle\psi|\varphi(1,1)| \chi\rangle \\
& =\lim _{w, \zeta \rightarrow 0}\left\langle 0\left|\psi^{\dagger}\left(\bar{w}^{-1}, w^{-1}\right) \varphi(1,1) \chi(\zeta, \bar{\zeta})\right| 0\right\rangle_{\mathbb{P}^{1}}
\end{aligned}
$$

Similarly, with $\varphi_{x}, \chi_{x} \in \mathcal{H}_{h_{x}, \bar{h}_{x}}$, four-point functions can be brought into the form

$$
\left\langle\varphi_{a}\left|\varphi_{b}(1) \varphi_{c}(z, \bar{z})\right| \varphi_{d}\right\rangle:=\lim _{w, \zeta \rightarrow 0}\left\langle 0\left|\varphi_{a}^{\dagger}\left(\bar{w}^{-1}, w^{-1}\right) \varphi_{b}(1,1) \varphi_{c}(z, \bar{z}) \varphi_{d}(\zeta, \bar{\zeta})\right| 0\right\rangle_{\mathbb{P}^{1}}
$$

They have the following expansion around $z=0$ :

$$
\begin{aligned}
& \left\langle\varphi_{a}\left|\varphi_{b}(1) \varphi_{c}(z, \bar{z})\right| \varphi_{d}\right\rangle \\
& \quad=\sum_{j} C\left(\varphi_{a}^{*}, \varphi_{b}, \psi_{j}\right) C\left(\psi_{j}^{*}, \varphi_{c}, \varphi_{d}\right) z^{h_{j}-h_{c}-h_{d}} \bar{z}^{h_{j}-\bar{h}_{c}-\bar{h}_{d}},
\end{aligned}
$$

where $\left\{\psi_{j}\right\}_{j}$ denotes a suitable orthonormal basis of $\mathcal{H}$.

Using the above characterization of $\psi^{\dagger}$, conformal invariance, and (A.6), one finds

$$
\forall \varphi, \chi, \psi \in \mathcal{H}: \quad \overline{C\left(\psi^{*}, \varphi, \chi\right)}=C\left(\chi^{*}, \varphi^{\dagger}, \psi\right) \stackrel{\substack{\text { if } \varphi \text { is quasi- } \\ \text { primary }}}{=} C\left(\chi^{*}, * \varphi, \psi\right) .
$$

Note that the OPE-coefficients involving only real states $* \varphi=\varphi, * \chi=\chi, * \psi=$ $\psi$ are always real. Moreover, using (A.6) one shows

$$
\forall n \in \mathbb{Z}: \quad L_{n}^{\dagger}=L_{-n} ; \quad \forall \chi, \psi \in \mathcal{H}: \quad\left(L_{n} \psi\right)^{*} \chi=\psi^{*}\left(L_{-n} \chi\right) .
$$


Since up to possible phases, $n$-point functions A.3 are invariant under permutations of the $\varphi_{i}\left(z_{i}, \bar{z}_{i}\right)$, the second and third arguments in $C(\cdot, \cdot, \cdot)$ can be interchanged, up to a phase and contributions of descendants to the OPE. However, the characterization (1.2.5) of primaries together with (A.11) ensures that every primary state is orthogonal to each descendant. Hence,

$$
\begin{aligned}
& \forall \varphi, \chi, \psi \in \mathcal{H} \text { with } \varphi \in \mathcal{H}_{h_{\varphi}, \bar{h}_{\varphi}}, \chi \in \mathcal{H}_{h_{\chi}, \bar{h}_{\chi}}, \psi \in \mathcal{H}_{h_{\psi}, \bar{h}_{\psi}} \cap \mathcal{H}^{\mathcal{V i r}}: \\
& C\left(\psi^{*}, \varphi, \chi\right)=(-1)^{h_{\chi}-\bar{h}_{\chi}+h_{\varphi}-\bar{h}_{\varphi}-h_{\psi}+\bar{h}_{\psi}} C\left(\psi^{*}, \chi, \varphi\right) .
\end{aligned}
$$

To define MODES associated to each $\varphi \in \mathcal{H}$, note that for all $h, \bar{h}, \mu, \bar{\mu}$, the space $\mathcal{H}_{h+\mu, \bar{h}+\bar{\mu}}$ is finite dimensional by (1.2.2), so we can set

$$
\begin{aligned}
& \forall \varphi \in \mathcal{H}, \forall \mu, \bar{\mu}, h, \bar{h} \in \mathbb{R}, \forall \chi \in \mathcal{H}_{h, \bar{h}}: \\
& \varphi_{\mu, \bar{\mu}} \chi \in \mathcal{H}_{h+\mu, \bar{h}+\bar{\mu}} \text { s. th. } \forall \psi \in \mathcal{H}_{h+\mu, \bar{h}+\bar{\mu}}: \psi^{*}\left(\varphi_{\mu, \bar{\mu}} \chi\right)=C\left(\psi^{*}, \varphi, \chi\right) .
\end{aligned}
$$

If $\varphi \in \mathcal{H}_{h, \bar{h}}$, then $\varphi_{h, \bar{h}}$ obeys $\varphi=\varphi_{h, \bar{h}} \Omega$. This gives

$$
\left[L_{0}, \varphi_{h, \bar{h}}\right]=h \varphi_{h, \bar{h}}, \quad\left[\bar{L}_{0}, \varphi_{h, \bar{h}}\right]=\bar{h} \varphi_{h, \bar{h}} .
$$

In general, all three-point functions in a CFT can be obtained as linear combinations of three-point functions of the primaries, acted on by differential operators. For example, if $\varphi \in \mathcal{H}_{h_{\varphi}, \bar{h}_{\varphi}}, \chi \in \mathcal{H}_{h_{\chi}, \bar{h}_{\chi}}, \psi \in \mathcal{H}_{h_{\psi}, \bar{h}_{\psi}}$, then

$$
C\left(\psi^{*}, L_{1} \varphi, \chi\right)=\left(h_{\psi}-h_{\varphi}-h_{\chi}\right) C\left(\psi^{*}, \varphi, \chi\right),
$$

and analogously for $\bar{L}_{1}$. On the other hand, analogously to A.9, all $n$-point functions of a CFT can be recovered from its OPE-constants. This imposes many consistency conditions on the latter. An important example for this is CROSSING SYMMETRY A.18 of four-point functions on the sphere.

Before discussing crossing symmetry, let us introduce W-ALGEBRAS, since we will use them to rewrite (A.9) in a slightly different way. Namely, for $\varphi \in \operatorname{ker}\left(\bar{L}_{0}\right)$ and $\chi \in \mathcal{H}_{h, \bar{h}}, \varphi_{\mu, \bar{\mu}} \chi \neq 0$ implies $(\mu, \bar{\mu})=(n, 0)$ with $n \in \mathbb{Z}$, and similarly for elements of $\operatorname{ker}\left(L_{0}\right)$ with $\mu, \bar{\mu}$ interchanged. The modes associated to states in $\operatorname{ker}\left(\bar{L}_{0}\right), \operatorname{ker}\left(L_{0}\right)$ generate a HOLOMORPHIC or ANTIHOLOMORPHIC W-ALGEBRA $\mathcal{W}^{*} \supset \mathcal{V}_{c}, \overline{\mathcal{W}}^{*} \supset \overline{\mathcal{V i r}}_{c}$ defined by

$$
\begin{aligned}
\mathcal{W}^{*} & :=\operatorname{span}_{\mathbb{C}}\left\{\varphi_{n, 0} \mid n \in \mathbb{Z}, \varphi \in \operatorname{ker}\left(\bar{L}_{0}\right)\right\} \\
& =\bigoplus_{n \in \mathbb{Z}} \mathcal{W}_{n}^{*}, \quad \mathcal{W}_{n}^{*}:=\left\{w \in \mathcal{W}^{*} \mid\left[L_{0}, w\right]=n w\right\},
\end{aligned}
$$

and analogously for $\overline{\mathcal{W}}^{*}$ or any subalgebra $\mathcal{W}$ of $\mathcal{W}^{*} \oplus \overline{\mathcal{W}}^{*}$. We suppose that $\mathcal{H}$ decomposes into a sum of tensor products of irreducible lowest weight representations $\mathcal{V}_{a}^{\mathcal{W}^{*}}, \overline{\mathcal{V}}_{\bar{a}}^{\overline{\mathcal{W}}^{*}}$ of the holomorphic and antiholomorphic W-algebras,

$$
\mathcal{H}=\bigoplus_{(a, \bar{a}) \in \mathcal{I}} \mathcal{V}_{a}^{\mathcal{W}^{*}} \otimes \overline{\mathcal{V}}_{\bar{a}}^{\overline{\mathcal{W}}^{*}}
$$


Moreover, the OPE determines the commutative associative product on the representation ring of $\mathcal{W}^{*} \otimes \overline{\mathcal{W}}^{*}$ which is known as FUSION:

$$
\left[\varphi_{a}\right] \bullet\left[\varphi_{b}\right]=\sum_{c} N_{a b}^{c}\left[\varphi_{c}\right]
$$

for conformal families $\left[\varphi_{a}\right]$ with $\varphi_{a} \in \mathcal{V}_{a}$ etc.

We now consider an orthonormal basis $\left\{\psi_{j}\right\}_{j \in \mathbb{N}}$ of primaries of a given CFT with respect to a subalgebra $\mathcal{W}$ of the holomorphic and antiholomorphic $\mathrm{W}$ algebra as in (1.2.5). Without loss of generality we can assume that $* \psi_{j}=\psi_{j}$ and $\psi_{j} \in \mathcal{H}_{h_{j}, \bar{h}_{j}}$ for all $j \in \mathbb{N}$. Moreover, let $\left\{\psi_{j}^{\{k, \bar{k}\}}\right\}_{k \in K, \bar{k} \in \bar{K}}$ with $K, \bar{K} \subset \oplus_{p} \mathbb{N}^{p}$ denote a basis of the descendants of $\psi_{j}$, which is $\left(L_{0}, \bar{L}_{0}\right)$-homogeneous, with bidegree of $\psi_{j}^{\{k, \bar{k}\}}$ given by $\left(h_{j}+|k|, \bar{h}_{j}+|\bar{k}|\right),|k|,|\bar{k}| \in \mathbb{N}$ for all $k \in K, \bar{k} \in \bar{K}$. For $a, b, j \in \mathbb{N}$ we set

$$
C_{a b}^{j}:=C\left(\psi_{j}^{*}, \psi_{a}, \psi_{b}\right), \quad \psi_{j}^{\{(),()\}}:=\psi_{j} .
$$

Then, there are constants $\beta_{a b}^{j\left\{k^{\prime}\right\}}, \bar{\beta}_{a b}^{j\left\{\bar{k}^{\prime}\right\}} \in \mathbb{R}$, such that

$$
\begin{aligned}
& \forall j \in \mathbb{N}, \forall k \in K, \bar{k} \in \bar{K}: \\
& \quad C\left(\left(\psi_{j}^{\{k, \bar{k}\}}\right)^{*}, \psi_{a}, \psi_{b}\right)=\sum_{k^{\prime}, \bar{k}^{\prime}} C_{a b}^{j} \beta_{a b}^{j\left\{k^{\prime}\right\}} \bar{\beta}_{a b}^{j\left\{\bar{k}^{\prime}\right\}} C\left(\left(\psi_{j}^{\{k, \bar{k}\}}\right)^{*}, \Omega, \psi_{j}^{\left\{k^{\prime}, \bar{k}^{\prime}\right\}}\right) .
\end{aligned}
$$

Here, $\beta_{a b}^{j\{()\}}=\bar{\beta}_{a b}^{j\{()\}}:=1$. Now, for all $a, b, c, d, j \in \mathbb{N}$ the CONFORMAL BLOCKS are given by

$$
\begin{aligned}
& \mathrm{f}_{j}\left[\begin{array}{ll}
a & b \\
c & d
\end{array}\right](z):=\sum_{k} \frac{\beta_{a b}^{j\{k\}}}{\sqrt{C_{c j}^{d}}} C\left(\psi_{d}^{*}, \psi_{c}, \psi_{j}^{\{k,()\}}\right) z^{h_{j}-h_{a}-h_{b}+|k|}, \\
& \overline{\mathrm{f}}_{j}\left[\begin{array}{ll}
a & b \\
c & d
\end{array}\right](\bar{z}):=\sum_{\bar{k}} \frac{\bar{\beta}_{a b}^{j\{\bar{k}\}}}{\sqrt{C_{c j}^{d}}} C\left(\psi_{d}^{*}, \psi_{c}, \psi_{j}^{\{(), \bar{k}\}}\right) \bar{z}^{h_{j}-\bar{h}_{a}-\bar{h}_{b}+|\bar{k}|} .
\end{aligned}
$$

Up to factors $z^{h_{j}-h_{a}-h_{b}}\left(\bar{z}^{h_{j}-\bar{h}_{a}-\bar{h}_{b}}\right)$, the conformal blocks are (anti-)meromorphic functions on $\mathbb{C}$ with poles at $0,1, \infty$. They encode the four-point functions of primaries by

$$
\left\langle\psi_{d}\left|\psi_{c}(1,1) \psi_{a}(z, \bar{z})\right| \psi_{b}\right\rangle=\sum_{j} C_{a b}^{j} C_{c j}^{d} \mathrm{f}_{j}\left[\begin{array}{ll}
a & b \\
c & d
\end{array}\right](z) \overline{\mathrm{f}}_{j}\left[\begin{array}{ll}
a & b \\
c & d
\end{array}\right](\bar{z}),
$$

and CROSSING SYMmetRY reads: for all $a, b, c, d \in \mathbb{N}$

$$
\begin{aligned}
\sum_{j} C_{a b}^{j} C_{c j}^{d} & \mathrm{f}_{j}\left[\begin{array}{ll}
a & b \\
c & d
\end{array}\right](z) \overline{\mathrm{f}}_{j}\left[\begin{array}{ll}
a & b \\
c & d
\end{array}\right](\bar{z}) \\
& =\sum_{j} C_{a d}^{j} C_{c j}^{b} \mathrm{f}_{j}\left[\begin{array}{ll}
a & d \\
c & b
\end{array}\right]\left(z^{-1}\right) \overline{\mathrm{f}}_{j}\left[\begin{array}{ll}
a & d \\
c & b
\end{array}\right]\left(\bar{z}^{-1}\right) z^{-2 h_{a}} \bar{z}^{-2 \bar{h}_{a}} .
\end{aligned}
$$




\section{B. $c=1$ Representation theory}

In this Appendix, let $\mathcal{C}=(\mathcal{H}, *, \Omega, T, \bar{T}, C)$ denote a unitary conformal field theory with $c=1$. We recall some basic facts about its representation content; see also Gab.

Since all known unitary conformal field theories at $c=1$ can be constructed with energy momentum tensor $T=\frac{1}{2}: j j$ : and $j$ a $\mathfrak{u}(1)$ current (which not necessarily is a field of the theory), it is convenient to use the Heisenberg algebra

$$
j(z)=\sum_{n=-\infty}^{\infty} a_{n} z^{n-1}, \quad \text { where } \quad\left[a_{n}, a_{m}\right]=m \delta_{n+m, 0} .
$$

Then all states in the pre-Hilbert space of every known theory $\mathcal{C}$ with central charge $c=1$ are obtained from the Fock space that we construct from appropriate polynomials in the $a_{n}, n>0$, acting on an appropriate subset of all lwvs of the Virasoro algebra. To build the latter it suffices to take states

$$
\begin{aligned}
|h, Q\rangle, \quad \text { such that } \quad L_{0}|h, Q\rangle & =h|h, Q\rangle, \text { with } h=\frac{Q^{2}}{2}, \\
a_{0}|h, Q\rangle & =Q|h, Q\rangle, \\
*(|h, Q\rangle) & =|h,-Q\rangle,
\end{aligned}
$$

as well as so-called twisted ground states with $h=\bar{h} \leq 1 / 16$, which we will not make use of in the following, however. We will always normalize the $|h, Q\rangle$ such that

$$
C\left(|h, Q\rangle^{*}, \Omega,|h, Q\rangle\right) \stackrel{1.2 .4}{=}\langle h,-Q \mid h, Q\rangle=1 .
$$

In a consistent theory, all left and right charges $(Q ; \bar{Q})$ are contained in a charge lattice. Namely, for every theory $\mathcal{C}$ there is a fixed $R \in \mathbb{R}^{+}$such that all $(Q ; \bar{Q})$ that may occur are given by

$$
(Q ; \bar{Q})=\frac{1}{\sqrt{2}}\left(m R+\frac{n}{R} ; m R-\frac{n}{R}\right), \quad m, n \in \mathbb{Z} .
$$

In a so-called CIRCLE THEORY AT RADIUS $R$, the pre-Hilbert space is just the entire Fock space built on the set of vacua $|Q ; \bar{Q}\rangle:=\left|\frac{Q^{2}}{2}, Q\right\rangle \otimes\left|\frac{\bar{Q}^{2}}{2}, \bar{Q}\right\rangle$ with all allowed values of $(Q ; \bar{Q})$. The $\mathfrak{s u}(2)_{1} \mathrm{WZW}$-model is the circle theory at radius $R=1$. All $|Q ; \bar{Q}\rangle$ are simple currents, and the leading terms in the OPE are given by

$$
C\left(\left|Q+Q^{\prime} ; \bar{Q}+\bar{Q}^{\prime}\right\rangle^{*},|Q ; \bar{Q}\rangle,\left|Q^{\prime} ; \bar{Q}^{\prime}\right\rangle\right)=(-1)^{(Q+\bar{Q})\left(Q^{\prime}-\bar{Q}^{\prime}\right) / 2},
$$

with all other OPE-constants vanishing. Equivalently,

$$
\begin{aligned}
|Q ; \bar{Q}\rangle \circledast\left|Q^{\prime} ; \bar{Q}^{\prime}\right\rangle & =\varepsilon\left((Q ; \bar{Q}),\left(Q^{\prime} ; \bar{Q}^{\prime}\right)\right)\left|Q+Q^{\prime} ; \bar{Q}+\bar{Q}^{\prime}\right\rangle \\
& =(-1)^{(Q+\bar{Q})\left(Q^{\prime}-\bar{Q}^{\prime}\right) / 2}\left|Q+Q^{\prime} ; \bar{Q}+\bar{Q}^{\prime}\right\rangle
\end{aligned}
$$

with notations as in (1.2.6). The COCYCLE FACTOR $\varepsilon$ introduces the appropriate phases. 
For central charge $c=1$, the character of a Virasoro irreducible representation with lowest weight vector of dimension $h$ generically is

$$
\chi_{h}^{g e n}(q)=\frac{1}{\eta(q)} q^{h} .
$$

But for $n=2 \sqrt{h} \in \mathbb{N}$, the representation contains a null vector at level $n+1$, namely [F-M-S, (8.34)]

$$
S_{n}\left|\frac{n^{2}}{4}\right\rangle:=\sum_{\substack{p_{i} \geq 1, p_{1}+\cdots+p_{k}=n+1}} \frac{(-1)^{n+1+k}}{\prod_{l=1}^{k-1}\left(p_{1}+\cdots+p_{l}\right)\left(n+1-p_{1}-\cdots-p_{l}\right)} L_{p_{1}} \cdots L_{p_{k}}\left|\frac{n^{2}}{4}\right\rangle,
$$

where $\left|\frac{n^{2}}{4}\right\rangle$ denotes the lowest weight vector of conformal weight $h=\frac{n^{2}}{4}, n \in \mathbb{N}$. Hence the character reduces to

$$
\chi_{\frac{1}{4} n^{2}}=\frac{1}{\eta(q)}\left(q^{n^{2} / 4}-q^{(n+2)^{2} / 4}\right) .
$$

In the following, we restrict attention to the holomorphic side only. The generic $\mathrm{W}$-algebra $\mathcal{W}$ of circle theories is generated by the $\mathfrak{u}(1)$ current $j$. The $\left|\frac{Q^{2}}{2}, Q\right\rangle$ are just the lowest weight vectors of irreducible representations $\mathcal{V}_{Q}^{\mathfrak{u}(1)}$ of $\mathcal{W}$ with characters

$$
X_{\sqrt{2} Q}=\frac{1}{\eta(q)} q^{\frac{Q^{2}}{2}},
$$

regardless of the value of $Q$. In particular, if $\sqrt{2} Q=n \in \mathbb{Z}$, by (B.9)

$$
X_{n}=\sum_{k=0}^{\infty} \chi_{\frac{1}{4}(|n|+2 k)^{2}}
$$

and the Fock space built on $\left|\frac{Q^{2}}{2}, Q\right\rangle$ contains infinitely many Virasoro irreducible representations with lowest weight vectors $|h, Q\rangle, h=\frac{Q^{2}}{2}+N, N=k(\sqrt{2}|Q|+$ $k), k \in \mathbb{N}$. Let

$$
|[n, m]\rangle:=\left|\frac{n^{2}}{4}, \frac{m}{\sqrt{2}}\right\rangle
$$

and let $\mathcal{V}_{[n, m]}$ denote the space of states in the irreducible representation of the Virasoro algebra with lwv $|[n, m]\rangle$ of norm 1. Note that e.g. for the circle theory at $R=1$ (the $\mathfrak{s u}(2)_{1}$ WZW model) each positive eigenvalue of $L_{0}$ is highly degenerate since this theory has an enhanced $\mathfrak{s u}(2)_{1}$ Kac-Moody algebra the zero modes of whose generators commute with $L_{0}$. More precisely,

$$
\mathcal{V}_{h=\frac{n^{2}}{4}}=\bigoplus_{m=-n, m \equiv n(2)}^{n} \mathcal{V}_{[n, m]} .
$$

All the representations $\mathcal{V}_{[n, m]}$ with $|m| \leq n, m \equiv n(2)$ have the same character $\chi_{\frac{1}{4} n^{2}}$ as in (B.9). Let $J_{ \pm}(z)$ denote the holomorphic fields creating $|Q ; \bar{Q}\rangle=$ $| \pm \sqrt{2} ; 0\rangle$ as in (A.7). Then we define

$$
Q_{ \pm}:=\int d z J_{ \pm}(z), \quad \text { i.e. }\left[Q_{+}, Q_{-}\right]=\sqrt{2} a_{0}=: 2 J_{0}, \quad\left[J_{0}, Q_{ \pm}\right]= \pm Q_{ \pm},
$$


the zero modes of $J_{ \pm}, J$ in the enhanced $\mathfrak{s u}(2)_{1}$ Kac-Moody algebra of the circle model at radius $R=1$. Since $\left[L_{n}, Q_{ \pm}\right]=0$ for all $n \in \mathbb{Z}$, from (B.10) together with $(\mathbb{B} .2)$ it follows that $|h, Q\rangle=\kappa Q_{\mp}|h, Q \pm \sqrt{2}\rangle$ for some $\kappa \in \mathbb{C}^{*}$ if $|h, Q \pm \sqrt{2}\rangle$ exists. More precisely, (B.10) inductively shows

$$
Q_{+}^{l} Q_{-}^{l}|[n, n]\rangle=\frac{l ! n !}{(n-l) !}|[n, n]\rangle,
$$

if the left hand side does not vanish. From our normalization $(\mathbb{B} .3)$ it now follows that

for $m, l \in \mathbb{N}$,

$$
|[n=m+2 l, m]\rangle=\sqrt{\frac{(n-l) !}{l ! n !}} Q_{-}^{l}|[n, n]\rangle, \text { and } Q_{ \pm}|[n, \pm n]\rangle=0 .
$$

In particular, $Q_{-}^{l}|[n, n]\rangle=0$ for $l>n$.

\section{The unitary Virasoro minimal models, their structure constants, and their $c \rightarrow 1$ limit}

The unitary diagonal Virasoro minimal model $\mathcal{M}_{m}:=\mathcal{M}(m, m+1)$ with $m \in$ $\mathbb{N}-\{0,1\}$ has central charge $c_{m}$ given by 4.1.1). Its irreducible representation $(r, s)$ of the Virasoro algebra has an lwv $|r, s\rangle_{m}$ with weight 4.1.3), and character

$$
\begin{gathered}
\chi_{(r, s)}^{m}(q)=\frac{q^{-\frac{c_{m}}{24}}}{\prod_{n}\left(1-q^{n}\right)}\left[q ^ { h _ { ( r , s ) } ^ { m } - } \sum _ { k = 1 } ^ { \infty } \left\{q^{h_{(r+(2 k-1) m,-s+m+1)}^{m}}+q^{h_{(r, 2 k(m+1)-s)}^{m}}\right.\right. \\
-q^{\left.\left.h_{(r+2 k m, s)}^{m}-q^{h_{(r, 2 k(m+1)+s)}^{m}}\right\}\right] .}
\end{gathered}
$$

Fusion reads

$$
\mathcal{V}_{\left(n^{\prime}, n\right)}^{m} \bullet \mathcal{V}_{\left(s^{\prime}, s\right)}^{m}=\bigoplus_{\substack{p^{\prime}=\left|n^{\prime}-s^{\prime}\right|+1, p^{\prime}+n^{\prime}+s^{\prime} \equiv 1(2)}}^{\min \left\{n^{\prime}+s^{\prime}-1,2 m+1-n^{\prime}-s^{\prime}\right\}} \bigoplus_{\substack{p=|n-s|+1, p+n+s \equiv 1(2)}}^{\min \{n+s-1,2 m-1-n-s\}} \mathcal{V}_{\left(p^{\prime}, p\right)}^{m} .
$$

The structure constants as in 4.1.8) are given by D-F3.

$$
\begin{aligned}
& C_{\left(n^{\prime}, n\right)\left(s^{\prime}, s\right)}^{\left(p^{\prime}, p\right)}=\mu_{l^{\prime}, l} \sqrt{\frac{a_{n^{\prime}, n^{\prime} s^{\prime}, s}}{a_{p^{\prime}, p}}} \\
& \times \prod_{i=0}^{l^{\prime}-2} \frac{\Gamma\left(s-s^{\prime}+1+i-y^{\prime}\left(s^{\prime}-1-i\right)\right) \Gamma\left(n-n^{\prime}+1+i-y^{\prime}\left(n^{\prime}-1-i\right)\right) \Gamma\left(p^{\prime}-p+1+i+y^{\prime}\left(p^{\prime}+1+i\right)\right)}{\Gamma\left(s^{\prime}-s-i+y^{\prime}\left(s^{\prime}-1-i\right)\right) \Gamma\left(n^{\prime}-n-i+y^{\prime}\left(n^{\prime}-1-i\right)\right) \Gamma\left(p-p^{\prime}-i-y^{\prime}\left(p^{\prime}+1+i\right)\right)} \\
& \times \prod_{j=0}^{l-2} \frac{\Gamma\left(s^{\prime}-s+2+j-l^{\prime}+y(s-1-j)\right) \Gamma\left(n^{\prime}-n+2+j-l^{\prime}+y(n-1-j)\right) \Gamma\left(p-p^{\prime}+2+j-l^{\prime}-y(p+1+j)\right)}{\Gamma\left(s-s^{\prime}-1-j+l^{\prime}-y(s-1-j)\right) \Gamma\left(n-n^{\prime}-1-j+l^{\prime}-y(n-1-j)\right) \Gamma\left(p^{\prime}-p-1-j+l^{\prime}+y(p+1+j)\right)} \\
& \quad=: \mu l_{l^{\prime}, l} \sqrt{\frac{a_{n^{\prime}, n} a_{s^{\prime}, s}}{a_{p^{\prime}, p}^{\prime}}} \tilde{C}_{\left(n^{\prime}, n\right)\left(s^{\prime}, s\right)}^{\left(p^{\prime}, p\right)},
\end{aligned}
$$


with

$$
\begin{aligned}
y & :=\frac{1}{m+1}, \quad y^{\prime}:=\frac{1}{m}, \quad l:=\frac{1}{2}(s+n-p+1), \quad l^{\prime}:=\frac{1}{2}\left(s^{\prime}+n^{\prime}-p^{\prime}+1\right), \\
\mu_{l^{\prime}, l} & :=(1-y)^{4\left(l^{\prime}-1\right)(l-1)} \prod_{i=1}^{l^{\prime}-1} \prod_{j=1}^{l-1} \frac{1}{(i-j+y j)^{2}} \prod_{i=1}^{l^{\prime}-1} \frac{\Gamma\left(i+i y^{\prime}\right)}{\Gamma\left(1-i-i y^{\prime}\right)} \prod_{j=1}^{l-1} \frac{\Gamma(j-j y)}{\Gamma(1-j+j y)} \\
& =(-1)^{(l-1)\left(l^{\prime}-1\right)}(1-y)^{4\left(l^{\prime}-1\right)(l-1)} \prod_{i=1}^{l^{\prime}-1} \frac{\Gamma\left(i+i y^{\prime}\right)}{\Gamma\left(1-i-i y^{\prime}\right)} \prod_{j=1}^{l-1} \frac{\Gamma\left(j-l^{\prime}+1-j y\right)}{\Gamma\left(l^{\prime}-j+j y\right)}, \\
a_{n^{\prime}, n} & :=\prod_{i=1}^{n^{\prime}-1} \prod_{j=1}^{n-1}\left(\frac{i-j+y(1+j)}{i-j+y j}\right)^{2} \prod_{i=1}^{n^{\prime}-1} \frac{\Gamma\left(i+i y^{\prime}\right) \Gamma\left(1-i-y^{\prime}(1+i)\right)}{\Gamma\left(1-i-i y^{\prime}\right) \Gamma\left(i+y^{\prime}(1+i)\right)} \prod_{j=1}^{n-1} \frac{\Gamma(j-j y) \Gamma(1-j+y(1+j))}{\Gamma(1-j+y j) \Gamma(j-y(1+j))} \\
& =\prod_{i=1}^{n^{\prime}-1} \frac{\Gamma\left(i+i y^{\prime}\right) \Gamma\left(1-i-y^{\prime}(1+i)\right)}{\Gamma\left(1-i-i y^{\prime}\right) \Gamma\left(i+y^{\prime}(1+i)\right)} \prod_{j=1}^{n-1} \frac{\Gamma\left(j-n^{\prime}+1-j y\right) \Gamma\left(n^{\prime}-j+y(1+j)\right)}{\Gamma\left(n^{\prime}-j+y j\right) \Gamma\left(j-n^{\prime}+1-y(1+j)\right)} .
\end{aligned}
$$

Note that $\mu_{l^{\prime}, l}, a_{n^{\prime}, n}$ and $\tilde{C}_{\left(n^{\prime}, n\right)\left(s^{\prime}, s\right)}^{\left(p^{\prime}, p\right)}$ are products of expressions

$$
\begin{aligned}
G(N, M, \epsilon):=\frac{\Gamma(1+N-M \epsilon)}{\Gamma(-N+M \epsilon)} & =(-1)^{N} \Gamma^{2}(1+N-M \epsilon) \frac{\sin (\pi M \epsilon)}{\pi} \\
& =\left((-1)^{N} \Gamma^{2}(-N+M \epsilon) \frac{\sin (\pi M \epsilon)}{\pi}\right)^{-1},
\end{aligned}
$$

where $N, M \in \mathbb{Z}$ and $\epsilon \in\{y, y\}$. We also have the following expansions for $m \rightarrow \infty$, to lowest order in $y=y^{\prime}+O\left(y^{2}\right)$ :

$$
\begin{aligned}
& G(N, M, \epsilon) \stackrel{y \rightarrow 0}{\sim}\left((-1)^{N} M y \Gamma^{2}\left(\left|\frac{1+\widetilde{\operatorname{sign}}(N)}{2}+N\right|\right)\right)^{\widetilde{\operatorname{sign}}(N)}=: y^{\widetilde{\operatorname{sign}}(N)} e(N, M), \\
& \frac{\Gamma(N+M \epsilon)}{\Gamma\left(N+M^{\prime} \epsilon^{\prime}\right)} \stackrel{y \rightarrow 0}{\sim}\left\{\begin{array}{ll}
\frac{M^{\prime}}{M} & \text { if } N \leq 0 \\
1 & \text { if } N>0
\end{array},\right.
\end{aligned}
$$

where $\widetilde{\operatorname{sign}}(N)=1$ for $N \geq 0, \widetilde{\operatorname{sign}}(N)=-1$ for $N<0$. Hence we obtain the lowest order expansions

$$
\begin{aligned}
& \mu_{l^{\prime}, l} \stackrel{y \rightarrow 0}{\sim} y^{\left|l-l^{\prime}\right|} \frac{(l-1) !\left(l^{\prime}-1\right) !}{\left(\left(\min \left\{l, l^{\prime}\right\}-1\right) !\right)^{2}} \prod_{i=1}^{l^{\prime}-1} \prod_{\substack{j=1, j \neq i}}^{l-1} \frac{1}{(i-j)^{2}} \prod_{i=1}^{l^{\prime}-1}(-1)^{i} \Gamma(i)^{2} \prod_{j=1}^{l-1}(-1)^{j+l^{\prime}} \Gamma(j)^{2} \\
& =: y^{\left|l-l^{\prime}\right|} \tilde{\mu}_{l^{\prime}, l}, \\
& a_{n^{\prime}, n} \stackrel{y \rightarrow 0}{\sim} \frac{\min \left\{n^{\prime}, n\right\}}{\max \left\{n^{\prime}, n\right\}}, \\
& \tilde{C}_{\left(n^{\prime}, n\right)\left(s^{\prime}, s\right)}^{\left(p^{\prime}, p\right)} \stackrel{y \rightarrow 0}{\sim} \tilde{A}_{\left(n^{\prime}, n\right)\left(s^{\prime}, s\right)}^{\left(p^{\prime}, p\right)} y^{\tilde{E}_{\left(n^{\prime}, n\right)\left(s^{\prime}, s\right)}^{\left(p^{\prime}, s\right)}} \\
& \tilde{E}_{\left(n^{\prime}, n\right)\left(s^{\prime}, s\right)}^{\left(p^{\prime} p\right)}:=k\left(s^{\prime}-s, l^{\prime}-2, l-2\right)+k\left(n^{\prime}-n, l^{\prime}-2, l-2\right)+k\left(p-p^{\prime}, l^{\prime}-2, l-2\right), \\
& k(x, a, b):=d(x, a)+d(-x, b)-2 g(x, a, b), \\
& d(x, a):=\max \{\min \{a+1, a+1-2 x\},-(a+1)\} \\
& =\frac{1}{2}(-x-|x|+|2 a+2-x-| x||), \\
& g(x, a, b):=\left(\min \left\{a-\frac{x}{2}, b+\frac{x}{2}\right\}-\frac{|x|}{2}+1\right) \Theta(a-x) \Theta(b+x) \\
& =\frac{1}{2}(-|x|+a+b+2-|a-b-x|) \Theta(a-x) \Theta(b+x),
\end{aligned}
$$


$\mathrm{SO}$

$$
k(x, a, b)=|a-b-x|-|x| \text { for } a, b \geq-1 .
$$

Moreover,

$$
\begin{aligned}
\tilde{A}_{\left(n^{\prime}, n\right)\left(s^{\prime}, s\right)}^{\left(p^{\prime}, p\right)}= & \prod_{i=0}^{l^{\prime}-2}\left\{e\left(s-s^{\prime}+i, s^{\prime}-1-i\right) e\left(n-n^{\prime}+i, n^{\prime}-1-i\right)\right. \\
& \left.e\left(p^{\prime}-p+i,-p^{\prime}-1-i\right)\right\} \prod_{j=0}^{l-2}\left\{e\left(s^{\prime}-s+1+j-l^{\prime},-s+1+j\right)\right. \\
& \left.e\left(n^{\prime}-n+1+j-l^{\prime},-n+1+j\right) e\left(p-p^{\prime}+1+j-l^{\prime}, p+1+j\right)\right\}
\end{aligned}
$$

Thus in the limit $m \rightarrow \infty$ we have

$$
C_{\left(n^{\prime}, n\right)\left(s^{\prime}, s\right)}^{\left(p^{\prime}, p\right)} \stackrel{y \rightarrow 0}{\sim} A_{\left(n^{\prime}, n\right)\left(s^{\prime}, s\right)}^{\left(p^{\prime}, p\right)} y_{\left(n^{\prime}, n\right)\left(s^{\prime}, s\right)}^{E^{\left(p^{\prime}, p\right)}}
$$

with

$$
\begin{aligned}
& A_{\left(n^{\prime}, n\right)\left(s^{\prime}, s\right)}^{\left(p^{\prime},\right)^{\prime}}=\left(\frac{\min \left\{n^{\prime}, n\right\} \min \left\{s^{\prime}, s\right\} \max \left\{p^{\prime}, p\right\}}{\max \left\{n^{\prime}, n\right\} \max \left\{s^{\prime}, s\right\} \min \left\{p^{\prime}, p\right\}}\right)^{1 / 2} \tilde{\mu}_{l^{\prime}, l} \tilde{A}_{\left(n^{\prime}, n\right)\left(s^{\prime}, s\right)}^{\left(p^{\prime}, p\right)}, \\
& E_{\left(n^{\prime}, n\right)\left(s^{\prime}, s\right)}^{\left(p^{\prime}, p\right)}=\left|l-l^{\prime}\right|+\tilde{E}_{\left(n^{\prime}, n\right)\left(s^{\prime}, s\right)}^{\left(p^{\prime}, p\right)} .
\end{aligned}
$$

Note that $A_{\left(n^{\prime}, n\right)\left(s^{\prime}, s\right)}^{\left(p^{\prime},\right)}$ never vanishes in the allowed regime $p^{\prime}+n^{\prime}+s^{\prime} \equiv p+n+s \equiv$ $1(2),\left|n^{(\prime)}-s^{(\prime)}\right|<p^{(\prime)}<n^{(\prime)}+s^{(\prime)}$. These constants obey

\section{Lemma C.1}

Given $\left(p^{\prime}, p\right),\left(n^{\prime}, n\right),\left(s^{\prime}, s\right)$ such that $A_{\left(n^{\prime}, n\right)\left(s^{\prime}, s\right)}^{\left(p^{\prime}, p\right)} \neq 0$, we have $E_{\left(n^{\prime}, n\right)\left(s^{\prime}, s\right)}^{\left(p^{\prime}, p\right)} \geq 0$. More precisely, with $\nu:=n^{\prime}-n, \sigma:=s^{\prime}-s, \pi:=p^{\prime}-p$,

$$
E_{\left(n^{\prime}, n\right)\left(s^{\prime}, s\right)}^{\left(p^{\prime}, p\right)}=0 \quad \Longleftrightarrow \quad|\pi| \in[\min \{|\sigma+\nu|,|\sigma-\nu|\}, \max \{|\sigma+\nu|,|\sigma-\nu|\}] .
$$

Proof:

Since $l^{\prime}-l=\frac{1}{2}(\sigma+\nu-\pi)$, from (C.5), (C.6), (C.8) we find

$$
\begin{aligned}
E_{\left(n^{\prime}, n\right)\left(s^{\prime}, s\right)}^{\left(p^{\prime}, p\right)}= & \frac{1}{2}|\sigma+\nu-\pi|+\frac{1}{2}|-\sigma+\nu-\pi|+\frac{1}{2}|\sigma-\nu-\pi|+\frac{1}{2}|\sigma+\nu+\pi| \\
& -|\sigma|-|\nu|-|\pi| \\
= & \max \{|\sigma+\nu|,|\pi|\}+\max \{|\sigma-\nu|,|\pi|\}-|\sigma|-|\nu|-|\pi| .
\end{aligned}
$$

Therefore,

$$
E_{\left(n^{\prime}, n\right)\left(s^{\prime}, s\right)}^{\left(p^{\prime}, p\right)}=\left\{\begin{aligned}
2 \max \{|\sigma|,|\nu|\}-|\sigma|-|\nu|-|\pi| & >0 \\
\text { if }|\pi| & <\min \{|\sigma+\nu|,|\sigma-\nu|\} \\
\max \{|\sigma+\nu|,|\sigma-\nu|\}-|\sigma|-|\nu| & =0 \\
\text { if }|\pi| & \in[\min \{|\sigma+\nu|,|\sigma-\nu|\} \\
|\pi|-|\sigma|-|\nu| & >0, \max \{|\sigma+\nu|,|\sigma-\nu|\}], \\
\text { if }|\pi| & >\max \{|\sigma+\nu|,|\sigma-\nu|\}
\end{aligned}\right.
$$

which proves the lemma. 
In Rem.4.1.7we explain how additional null vectors in the limit $\mathcal{M}_{\infty}$ of unitary Virasoro minimal models can be scaled up without introducing divergences in three-point functions. In fact, Lemma C.1 can be used in order to extend the example of scaling up null vectors given in G-R-W Sect. 3.1.1] by a direct calculation:

\section{Lemma C.2}

All vectors $L_{1}|r, r\rangle_{\infty}, r>1$, can be scaled up to non-vanishing norm without introducing divergences in the OPE-constants $C\left(\left|p^{\prime}, p\right\rangle_{\infty}^{*}, L_{1}|r, r\rangle_{\infty},\left|s^{\prime}, s\right\rangle_{\infty}\right)$.

Proof:

By 4.1.13), a normalization of $L_{1}|r, r\rangle_{\infty}$ to non-vanishing but finite norm is given by

$$
D_{r, r, 1}^{m}:=\lim _{m \rightarrow \infty}(m+1) L_{1}|r, r\rangle_{m}
$$

i.e. we set

$$
\widetilde{f}_{m}^{j}\left(D_{r, r, 1}^{m}\right):=D_{r, r, 1}^{j} .
$$

Note that for finite $m$, (C.2) shows that $C_{(r, r)\left(s^{\prime}, s\right)}^{\left(p^{\prime}, p\right)}$ is only non-vanishing if $\frac{1}{2}(r+$ $\left.s^{(\prime)}-1-p^{(\prime)}\right) \in\left\{0, \ldots, \min \left\{r, s^{(\prime)}\right\}-1\right\}$, hence we restrict to $\operatorname{such} p, p^{\prime}$. By (A.14) we find

$$
\begin{aligned}
C_{D_{r, r, 1}\left(s^{\prime}, s\right)}^{\left(p^{\prime}, p\right)} & \stackrel{m \rightarrow \infty}{\sim}(m+1)\left(h_{\left(p^{\prime}, p\right)}^{m}-h_{(r, r)}^{m}-h_{\left(s^{\prime}, s\right)}^{m}\right) C_{(r, r)\left(s^{\prime}, s\right)}^{\left(p^{\prime}, p\right)} \\
& \stackrel{\text { C.7 }}{\sim}(m+1)^{1-E_{(r, r)\left(s^{\prime}, s\right)}^{\left(p^{\prime}, p\right)}}\left(h_{\left(p^{\prime}, p\right)}^{m}-h_{(r, r)}^{m}-h_{\left(s^{\prime}, s\right)}^{m}\right) A_{(r, r)\left(s^{\prime}, s\right)}^{\left(p^{\prime}, p\right)} .
\end{aligned}
$$

Therefore, if $E_{(r, r)\left(s^{\prime}, s\right)}^{\left(p^{\prime}, p\right)} \geq 1$, the assertion follows directly from the convergence of each term in the latter expression.

On the other hand, for $p^{\prime}, p$ in the range given above, by Lemma C.1 we have $E_{(r, r)\left(s^{\prime}, s\right)}^{\left(p^{\prime}, p\right)}=0$ iff $\left|p^{\prime}-p\right|=\left|s^{\prime}-s\right|$. Hence in this case

$$
\begin{aligned}
& C_{D_{r, r, 1}\left(s^{\prime}, s\right)}^{\left(p^{\prime}, p\right)} \stackrel{m \rightarrow \infty}{\sim}(m+1)\left(h_{\left(p^{\prime}, p\right)}^{m}-h_{(r, r)}^{m}-h_{\left(s^{\prime}, s\right)}^{m}\right) C_{(r, r)\left(s^{\prime}, s\right)}^{\left(p^{\prime}, p\right)} \\
& \stackrel{4.1 .3]}{=}\left\{\frac{m+1}{4 m}\left(\left(p^{\prime}\right)^{2}-p^{2}-\left(s^{\prime}\right)^{2}+s^{2}\right)+\mathcal{O}\left(\frac{1}{m}\right)\right\} A_{(r, r)\left(s^{\prime}, s\right)}^{\left(p^{\prime}, p\right)}
\end{aligned}
$$

remains finite, too.

\section{References}

A-G-S-G. Alvarez-Gaumé, L., Sierra, G., Gomez, C.: Topics in conformal field theory. In: Physics and mathematics of strings, World Sci. Publishing, Teaneck, NJ, 1990, pp. 16-184

A-G-M. $\quad$ Aspinwall, P.S., Greene, B.R., Morrison, D.R.: Calabi-Yau moduli space, mirror manifolds and spacetime topology change in string theory. Nucl. Phys. B416, 414-480 (1994); hep-th/9309097

A-M. Aspinwall, P.S., Morrison, D.R.: String theory on K3 surfaces. In: Greene, B.,

B-P-Z Yau, S.T. (eds): Mirror symmetry, vol. II, 1994, pp. 703-716; hep-th/9404151

in two-dimensional quantum field theory. Nucl. Phys. B241, 333-380 (1984)

B-G-V. Berline, N., Getzler, E., Vergne, M.: Heat kernels and Dirac operators. SpringerVerlag, Berlin Heidelberg New York, 1992 
Bo. Bourbaki, N.: Éléments de mathématique. Théorie des ensembles. Hermann, Paris, 1970

B-N. Brungs, D., Nahm, W.: The associative algebras of conformal field theory. Lett. Math. Phys. 47 no. 4, 379-383 (1999); hep-th/9811239

C-I-Z1. Capelli, A., Itzykson, C., Zuber, J.B.: The A-D-E classification of minimal and $A_{1}^{(1)}$ conformal invariant theories. Commun. Math. Phys. 113, 1-26 (1987)

C-I-Z2. Cappelli, A., Itzykson, C., Zuber, J.B.: Modular invariant partition functions in two dimensions. Nucl. Phys. B280, 445-465 (1987)

C-E-N-T. Casher, A., Englert, F., Nicolai, H., Taormina, A.: Consistent superstrings as solutions of the $D=26$ bosonic string theory. Phys. Lett. B162, 121-126 (1985)

C-G1. Cheeger, J., Gromov, M.: Collapsing Riemannian manifolds while keeping their curvature bounded. I. J. of Diff. Geometry 23 no. 3, 309-346 (1986)

C-G2. Cheeger, J., Gromov, M.: Collapsing Riemannian manifolds while keeping their curvature bounded. II. J. of Diff. Geometry 32 no. 1, 269-298 (1990)

Co1. Connes, A.: Noncommutative differential geometry. Inst. Hautes Études Sci. Publ. Math. no. 62, 257-360 (1985)

Co2. Connes, A.: Géométrie non commutative. InterEditions, 1990

Co3. Connes, A.: Gravity coupled with matter and the foundation of non-commutative geometry. Commun. Math. Phys. 182 no. 1, 155-176 (1996)

D-V-V. Dijkgraaf, R., Verlinde, E., Verlinde, H.: $c=1$ Conformal field theories on Riemann surfaces. Commun. Math. Phys. 115, 646-690 (1988)

D-V-V-V. Dijkgraaf, R., Vafa, C., Verlinde, E., Verlinde, H.: The operator algebra of orbifold models. Commun. Math. Phys. 123, 485-543 (1989)

D-F-M-S. Dixon, L.J., Friedan, D., Martinec, E., Shenker, S.: The conformal field theory of orbifolds. Nucl. Phys. B282, 13-73 (1987)

Do. Dold, A.: Lectures on algebraic topology. Die Grundlehren der mathematischen Wissenschaften, vol. 200, Springer-Verlag, Berlin-Heidelberg-New York, 1972

D-F1. Dotsenko, V.S., Fateev, V.A.: Conformal algebra and multipoint correlation functions in 2D statistical models. Nucl. Phys. B240, 312-348 (1984)

D-F2. Dotsenko, V.S., Fateev, V.A.: Four-point correlation functions and the operator algebra in $2 \mathrm{D}$ conformal invariant theories with central charge $C \leq 1$. Nucl. Phys. B251, 691-769 (1985)

D-F3. Dotsenko, V.S., Fateev, V.A.: Operator algebra of two-dimensional conformal field theories with central charge $c \leq 1$. Phys. Lett. B154, 291-295 (1985)

D-H-V-W. Dixon, L.J., Harvey, J., Vafa, C., Witten, E.: Strings on orbifolds II. Nucl. Phys. B274, 285-314 (1986)

F-F. $\quad$ Feigin, B.L., Fuks, D.B.: Moscow preprint. 1983

Fe1. Felder, G.: BRST approach to minimal models. Nucl. Phys. B317, 215-236 (1989)

Fe2. $\quad$ Felder, G.: Erratum: BRST approach to minimal models. Nucl. Phys. B324, 548 (1989)

F-R. $\quad$ Förste, S., Roggenkamp, D.: Current-current deformations of conformal field theories, and WZW models. JHEP 0305, 71 (2003); hep-th/0304234

F-M-S. Di Francesco, P., Mathieu, P., Sénéchal, D.: Conformal field theory. Graduate Texts in Contemporary Physics, Springer-Verlag, New York Berlin Heidelberg Barcelona Hong Kong London Milan Paris Singapore Tokyo, 1996

Fr-S. $\quad$ Fredenhagen, S., Schomerus, V.: D-branes in coset models. JHEP 02, 005 (2002); hep-th/0111189

F-G. Fröhlich, J., Gawędzki, K.: Conformal field theory and geometry of strings. In: Mathematical quantum theory. I. Field theory and many-body theory (Vancouver, BC, 1993). Amer. Math. Soc., Providence, RI, 1994, pp. 57-97; hep-th/9310178

F-G-R. $\quad$ Fröhlich, J., Grandjean, O., Recknagel, A.: Supersymmetric quantum theory, noncommutative geometry, and gravitation. In: Symétries quantiques (Les Houches, 1995). North-Holland, Amsterdam, 1998, pp. 221-385; hep-th/9706132

Fu-S. $\quad$ Fuchs, J., Schweigert, C.: WZW fusion rings in the limit of infinite level. Commun. Math. Phys. 185, 641-670 (1997); hep-th/9609124

Gab. Gaberdiel, M.R. : D-branes from conformal field theory. Proceedings of the Workshop on the Quantum Structure of Spacetime and the Geometric Nature of Fundamental Interactions, Corfu, Greece, 13-20 September 2001; hep-th/0201113

G-G. Gaberdiel, M.R., Goddard, P.: Axiomatic conformal field theory. Commun. Math. Phys. 209, 549-594 (2000); hep-th/9810019

G-N. Gaberdiel, M.R., Neitzke, A.: Rationality, quasirationality and finite W-algebras. Commun. Math. Phys. 238, 305-331 (2003); hep-th/0009235 
Gaw. Gawędzki, K.: Boundary WZW, G/H, G/G and CS theories. Annales Henri Poincare 3, 847-881 (2002); hep-th/0108044

Gi. Ginsparg, P.: Applied conformal field theory. Lectures given at the Les Houches Summer School in Theoretical Physics 1988 (Les Houches, France), pp. 1-168

G-R-W. Graham, K., Runkel, I., Watts, G.M.T.: Minimal model boundary flows and $c=1$ CFT. Nucl. Phys. B608, 527-556 (2001); hep-th/0101187

Gr. $\quad$ Gross, M. : Topological mirror symmetry. Invent. Math. 144 no. 1, 75-137 (2001); math.AG/9909015

He. Henrici, P.: Essentials of numerical analysis with pocket calculator demonstrations. John Wiley \& Sons Inc., New York, 1982

I-S-Z. Itzykson, C., Saleur, H., Zuber, J.-B. (eds): Conformal invariance and applications to statistical mechanics. World Scientific, Singapore, 1988

Ka. Kadanoff, L.P.: Multicritical behaviour at the Kosterlitz-Thouless Critical Point. Ann. Physics 120, 39-71 (1979)

K-S. $\quad$ Kontsevich, M., Soibelman, Y.: Homological mirror symmetry and torus fibrations. In: Symplectic geometry and mirror symmetry (Seoul, 2000). World Sci. Publishing, River Edge, NJ, 2001, pp. 203-263; math.SG/0011041

Lo. $\quad$ Lord, S.: Riemannian Geometries; math-ph/0010037

M-M-S. Maldacena, J., Moore, G., Seiberg, N.: Geometrical interpretation of D-branes in gauged WZW models. JHEP 07, 046 (2001); hep-th/0105038

M-S1. Moore, G., Seiberg, N.: Classical and quantum conformal field theory. Commun. Math. Phys. 123, 177-253 (1989)

M-S2. Moore, G., Seiberg, N.: Naturality in conformal field theory. Nucl. Phys. B313, $16(1989)$

Mo. $\quad$ Morrison, D.R.: Where is the large radius limit? In: Halpern, M.B., et. al (eds): Proceedings of Strings '93, Berkeley, CA, USA, May 24-29, 1993. World Scientific, Singapore, pp. 311-315; hep-th/9311049

Nah. Nahm, W.: On quasi-rational conformal field theories. Nucl. Phys. B (Proc. Suppl.) 49, 107-114 (1996)

Nar. $\quad$ Narain, K.S.: New heterotic string theories in uncompactified dimensions $<10$. Phys. Lett. B169, 41-46 (1986)

R-S-Z. Ranganathan, K., Sonoda, H., Zwiebach, B.: Connections on the state space over conformal field theories. Nucl. Phys. B414, 405-460 (1994); hep-th/9304053

R-R-S. Recknagel, A., Roggenkamp, D., Schomerus, V.: On relevant boundary perturbations of unitary minimal models. Nucl. Phys. B588, 552-564 (2000); hep-th/ 0003110

Re. $\quad$ Rennie, A.: Commutative geometries are spin manifolds. Rev. Math. Phys. 13 no. 4, 409-464 (2001); math-ph/9903021

R-W1. Runkel, I., Watts, G.M.T.: A non-rational CFT with $\mathrm{c}=1$ as a limit of minimal models. Proceedings of the Workshop on the Quantum Structure of Spacetime and the Geometric Nature of Fundamental Interactions, Corfu, Greece, 13-20 September 2001; hep-th/0107118

R-W2. Runkel, I., Watts, G.M.T.: A non-rational CFT with central charge 1. Fortsch. Phys. 50, 959-965 (2002); hep-th/0201231

Sch. Schwinger, J.: The theory of quantized fields. II. In: Schwinger, J. (ed): Selected papers on quantum electrodynamics. Dover publications Inc., 1958, pp. 356-371

S-Y-Z. Strominger, A., Yau, S.-T., Zaslow, E.: Mirror symmetry is T-duality. Nucl. Phys. B479, 243-259 (1996); hep-th/9606040

V-W. Vafa, C., Witten, E.: On orbifolds with discrete torsion. J. Geom. Phys. 15, 189214 (1995); hep-th/9409188

Wi. Widder, D.V.: The Laplace Transform. Princeton Mathematical Series, v. 6, Princeton University Press, Princeton, NJ, 1941

Za1. Zamolodchikov, A.B.: Conformal symmetry and multicritical points in two-dimensional quantum field theory. Sov. J. Nucl. Phys. 44, 529-533 (1986), in Russian; reprinted in I-S-Z

Za2. Zamolodchikov, A.B.: Renormalization group and perturbation theory near fixed points in two-dimensional field theory. Sov. J. Nucl. Phys. 46, 1090-1096 (1987) Zhu, Y.: Modular invariance of characters of vertex operator algebras. J. Amer. Math. Soc. 9, 237-302 (1996) 